INTER NATIONAL MONETARY FUND

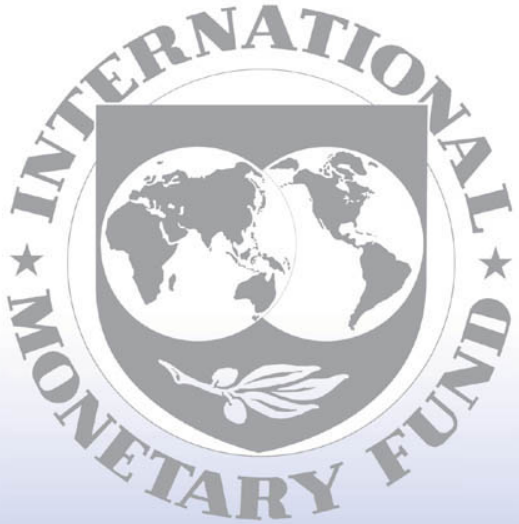

Staff

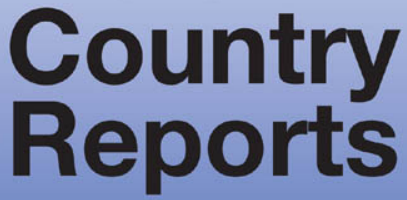




\section{Rwanda: Second and Third Reviews Under the Three-Year Arrangement Under the Poverty Reduction and Growth Facility, Request for Waiver of Performance Criteria, and Request for Additional Interim Assistance Under the Enhanced HIPC Initiative- Staff Report; Staff Statements; Press Release on the Executive Board Discussion; and Statement by the Executive Director for Rwanda}

In the context of the second and third reviews under the three-year arrangement under the Poverty Reduction and Growth Facility, request for waiver of performance criteria, and request for additional interim assistance under the enhanced HIPC Initiative with Rwanda, the following documents have been released and are included in this package:

- the staff report for the second and third reviews under the three-year arrangement under the Poverty Reduction and Growth Facility, request for waiver of performance criteria, and for additional interim assistance under the enhanced HIPC Initiative, prepared by a staff team of the IMF, following discussions that ended on February 23, 2004, with the officials of Rwanda on economic developments and policies. Based on information available at the time of these discussions, the staff report was completed on May 24, 2004. The views expressed in the staff report are those of the staff team and do not necessarily reflect the views of the Executive Board of the IMF.

- $\quad$ staff statements of June 8, 2004 updating information on recent developments.

- $\quad$ a Press Release summarizing the views of the Executive Board as expressed during its June 9, 2004 discussion of the staff report that completed the requests and reviews.

- $\quad$ a statement by the Executive Director for Rwanda.

The documents listed below have been or will be separately released.

Joint Staff Assessment of the Poverty Reduction Strategy Paper Progress Report

Letter of Intent sent to the IMF by the authorities of Rwanda*

Memorandum of Economic and Financial Policies by the authorities of Rwanda*

Poverty Reduction Strategy Paper Progress Report

Technical Memorandum of Understanding*

* May also be included in Staff Report

The policy of publication of staff reports and other documents allows for the deletion of market-sensitive information.

To assist the IMF in evaluating the publication policy, reader comments are invited and may be sent by e-mail to publicationpolicy@imf.org.

Copies of this report are available to the public from

International Monetary Fund $\bullet$ Publication Services

$70019^{\text {th }}$ Street, N.W. • Washington, D.C. 20431

Telephone: (202) 623-7430 • Telefax: (202) 623-7201

E-mail: publications@imf.org • Internet: http://www.imf.org

Price: $\$ 15.00$ a copy

International Monetary Fund

Washington, D.C. 
This page intentionally left blank

CInternational Monetary Fund. Not for Redistribution 
INTERNATIONAL MONETARY FUND

\title{
RWANDA
}

\section{Second and Third Reviews Under the Three-Year Arrangement Under the Poverty Reduction and Growth Facility, Request for Waiver of Performance Criteria, and Request for Additional Interim Assistance Under the Enhanced HIPC Initiative}

\author{
Prepared by the African Department \\ (In collaboration with other departments)
}

Approved by Juan Carlos Di Tata and Mark Plant

May 24, 2004

\begin{abstract}
A three-year Poverty Reduction and Growth Facility (PRGF) arrangement for SDR 4 million was approved on August 12, 2002. The first tranche of SDR 574,000 was disbursed upon approval, and a further SDR 571,000 was disbursed following the completion of the first review, on June 13, 2003. Upon the completion of the second and third reviews, Rwanda will be eligible to draw an amount equivalent to SDR 1.14 million (1.4 percent of quota). As set out in Appendix I, Attachment I, the forthcoming fourth review is proposed to be linked to the performance criteria for end-June 2004.
\end{abstract}

Discussions for the second and third reviews under the PRGF took place in Kigali during November 1229, 2003 and February 2-11, 2004, and were followed by discussions at headquarters during February 19-23, 2004. The staff teams headed by Mr. Meyers consisted of Messrs. Christensen, Blavy (both AFR), and Rother (EP-PDR) for the November 2003 mission, and Messrs. Hauner (EP-AFR), and Rother (EP-PDR) for the February 2004 mission. Mr. Farah, the Resident Representative in Kigali assisted the missions. Mr. Rutayisire (Alternative Executive Director for Rwanda) participated in the November 2003 mission.

On June 13, 2003, the Executive Board completed the first review of the three-year arrangement under the PRGF. Executive Directors commended Rwanda's strong commitment to macroeconomic stability. They stressed the crucial role of economic diversification in reducing external vulnerability, and attached a high priority to implementing the policies set out in the Poverty Reduction Strategy Paper (PRSP) for achieving external sustainability (BUFF 03/92).

Rwanda could reach the completion point under the Enhanced Initiative for Heavily Indebted Poor Countries (HIPC Initiative) at end-2004, following completion of the fourth review under the PRGF arrangement. Since the country reached the decision point in December 2000, it has been benefiting from debt relief from its multilateral and bilateral creditors. Paris Club creditors have been requested to extend the deadline for reaching bilateral agreements to June 30, 2004. Rwanda is also continuing efforts to reach rescheduling agreements with all remaining non-Paris Club creditors. The IMF did not provide HIPC Initiative Interim assistance in 2003. Interim assistance under the HIPC Initiative is being requested for the period June 9, 2004 to June 8, 2005. 
I. Introduction

II. Recent Developments and Program Performance................................................. 4

III. Policy Discussions and Revised Program for 2004 .............................................. 9

A. Poverty Reduction Strategy and Growth ........................................................

B. Fiscal Policy ........................................................................................

C. Monetary Policy .....................................................................................

D. External Sector Policies ........................................................................

E. Structural Reforms................................................................................

F. Governance and Safeguards ................................................................

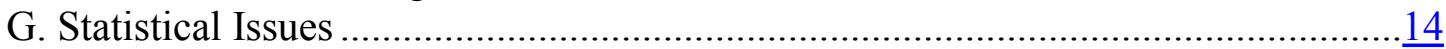

H. Program Monitoring............................................................................

IV. Staff Appraisal ...........................................................................................

Boxes

1. Debt Sustainability ..................................................................................

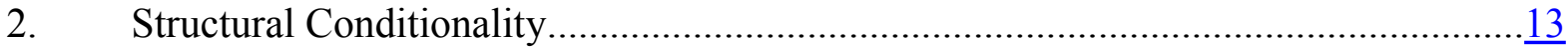

Figures

1. Real GDP Growth, 1996-2006 .......................................................................

2. Real and Nominal Exchange Rates, January 1999-December 2003 ..........................5

3. Price Developments, January 2000-February 2004 .............................................. $\frac{5}{6}$

4. Broad Money and Reserve Money December 2000-December 2003........................

Tables

1. Selected Economic and Financial Indicators, 2001-06 .......................................23

2. Operations of the Central Government, 2001-06............................................24

3. Monetary Survey, 2001-04 .......................................................................... 26

4. Balance of Payments, 2001-06 .......................................................................

5. External Financing Requirement and Sources, 2001-06......................................29

6. External Public Debt and Debt Service, 2001-06 ...............................................

Appendices

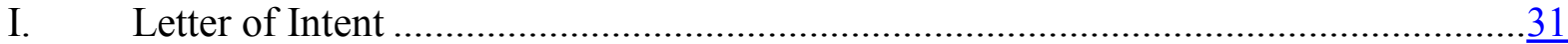

Attachment I: Memorandum of Economic and Financial Policies of the

Government of Rwanda for 2003 ........................................................... 33

Attachment II: Technical Memorandum of Understanding Between the

Government of Rwanda and the International Monetary Fund .......................

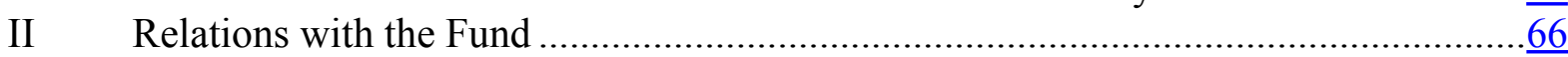

III. IMF-Bank Relations Annex ...............................................................

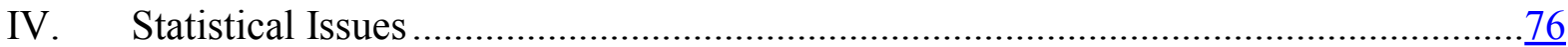


V. Work Program-Proposed Phasing of Disbursements Under the PRGF Arrangement, 2002-05

$\underline{80}$

VI. Safeguards Assessment-Summary of Conclusions ………………………............

VII. Millennium Development Goals ....................................................................... 


\section{INTRODUCTION}

1. In the ten years since the $\mathbf{1 9 9 4}$ genocide, Rwanda has reestablished the critical elements of an effective state. This accomplishment reflects the creation and reform of institutions, the implementation of market-based economic policies, a substantial rehabilitation of infrastructure, the reconstitution of required work skills, and the development of poverty-oriented social programs. The adoption of a new constitution in May 2003, followed by a presidential election in August, and legislative polls in SeptemberOctober set in place the political foundations that will support Rwanda's economic and financial development.

2. Rwanda addressed a wide range of challenges during 2003. It maintained its commitment to peace in the Great Lakes region, continued with the process of demobilization and reintegration, moved forward with community-based hearings for the genocide-accused, and carried on with the decentralization of government. This extraordinary agenda clearly stretched Rwanda's management capacity. Macroeconomic policy performance, as noted in the attached memorandum of economic and financial policies (MEFP) and in the paragraphs below, fell short of the standard that had been maintained under preceding programs.

\section{Rwanda's development partners have strengthened their coordination and} support efforts in the context of the Strategic Partnership for Africa. Rwanda's principal donors have committed to increase budget support in 2004 by 3.7 percentage points of GDP relative to earlier projections, mostly in the form of grants. During the second half of 2004, the World Bank's Executive Board is expected to consider a three-year Poverty Reduction Support Credit (PRSC) that would provide US\$70-US\$80 million per annum in budgetary support during 2005-07. The prudent management of these inflows will be key to Rwanda's development strategy.

\section{RECENT DEVELOPMENTS AND PROGRAM PERFORMANCE}

4. Macroeconomic performance under the an extended period characterized by a strong expansion of economic activity, real GDP growth is estimated by the staff to have slowed to 0.9 percent in 2003 (Figure 1). ${ }^{1}$ Following exceptionally strong growth in 2002 , food production fell well below the expected level, owing to poor rains. A 15 percent increase in 2003 program fell short of expectations. After

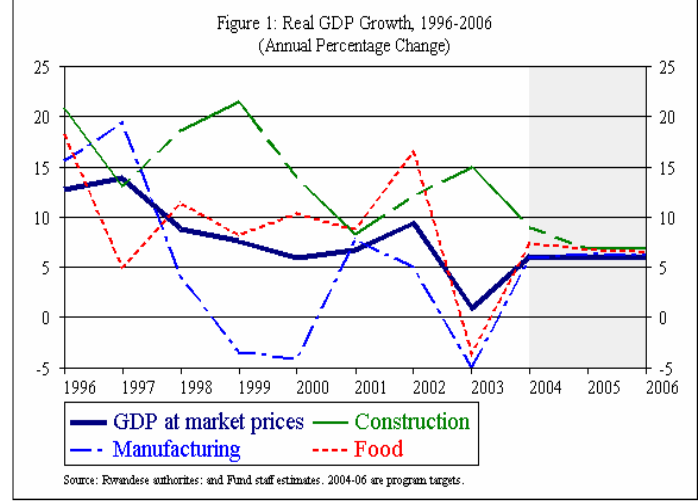

\footnotetext{
${ }^{1}$ This figure is 2.3 percentage points below the authorities' estimate, reflecting the staff's evaluation that real growth in fruit and vegetable output was no more than 30 percent, compared with the authorities' estimate of 100 percent.
} 
construction moderated the slowdown in economic activity. At the same time, a drop in export earnings driven by exogenous factors, along with a construction-related increase in demand for foreign exchange, led to a 20 percent depreciation of the Rwanda franc in real effective terms (Figure 2). Drought-induced food price increases and the incipient pass-through of exchange rate depreciation resulted in an increase in consumer price inflation to 7.7 percent (5.5 percent excluding food) by year's end (Figure 3).

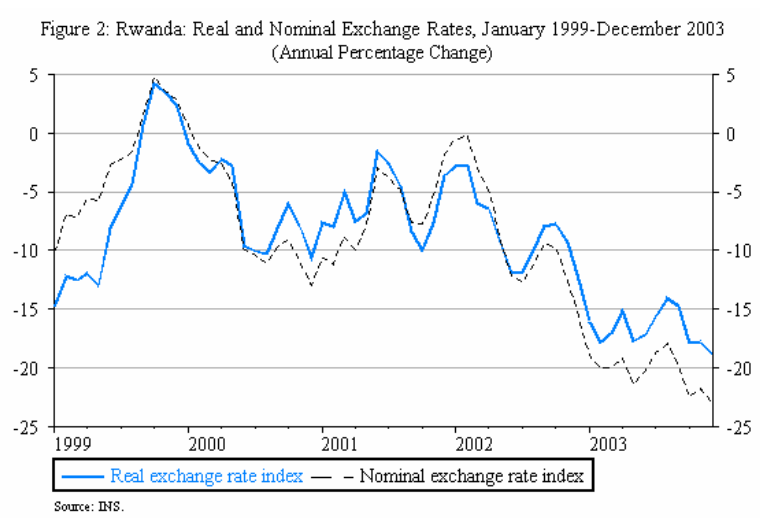

\section{In acknowledging program} slippages during 2003, the authorities pointed to the critical significance of the political transition, which led to unbudgeted outlays on the elections and goods and services (MEFP, paragraph 6). Six quantitative performance criteria and all structural performance criteria were met at end-June 2003, and three quantitative performance criteria were narrowly missed. Policy performance, however, weakened significantly during the second half of 2003.

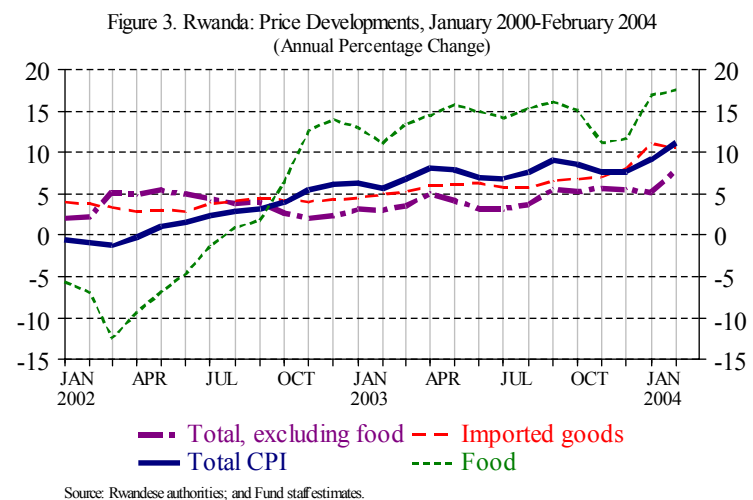

As a result, six quantitative performance criteria and three structural performance criteria for end-December 2003 and early 2004 were not observed (MEFP, Appendix I, Attachment I, Tables 1 and 2).

6. The missed quantitative performance criteria for end-December 2003 resulted from unprogrammed government outlays on the elections (paragraph 8), the financing of a hotel project (paragraph 10), and delayed donor disbursements (paragraph 9). To correct for these slippages, the 2004 program includes a substantial reduction in net credit to government from the banking system, slower reserve money growth, and a fiscal program that incorporates contingent cuts to assure that the government's domestic fiscal balance remains consistent with macroeconomic objectives. Data on government financial operations through end-March 2004 show that revenue and expenditure performance are in line with end-June performance criteria. Payment orders were issued during January-April 2004 to eliminate the stock of domestic arrears that had accumulated at end-2003. As of April 30, 2004, net central bank credit to government was below the projected requirement and, as a result, reserve money growth was lower and the net foreign assets of the NBR higher than had been targeted. The missed performance criteria on new non-concessional external debt, which resulted from an energy rehabilitation loan with a grant element of 47 percent, compared to the 50 percent floor set in the program (MEFP paragraph 13). The financing for this project 
will be brought to the required concessionality level by end-June 2004. These developments demonstrate that action has been taken to correct missed quantitative performance criteria. Based on the above, the authorities request that the nonobservance of the quantitative performance criteria be waived.

7. On the structural side, performance criteria on the revision of the tax law and preparation of the financial instructions for more effective expenditure management were met. However, the structural performance criteria for submission of a revised investment code, issuance of financial instructions for more effective expenditure management, and incorporation of tax incentives into the structure of the income tax were not observed. Moreover, some structural benchmarks were missed. These slippages were mainly associated with the authorities' limited pool of adequately trained staff. The authorities set firm dates for the completion of the missed criteria (MEFP paragraphs 37 and 40). Implementation is underway. Signaling a strengthened resolve to advance the structural agenda, the authorities committed to implementing three prior actions for the completion of the second and third reviews under the PRGF arrangement (Box 2). Based on the above, the authorities request that the nonobservance of the structural performance criteria be waived.

8. Fiscal performance, broadly on target through end-June 2003, weakened during the second half of 2003. ${ }^{2}$ For the year as a whole, domestic revenues, which increased from 12.2 percent of GDP in 2002 to 13.5 percent in 2003, slightly exceeded the programmed level. However, there were expenditure overruns on elections and goods and services, and the government failed to implement expenditure reductions to offset unrealized grants for special health operations. As a result, the domestic fiscal deficit reached 5.5 percent of GDP in 2003, compared with the program's target of 2.6 percent of GDP (after adjusters). Lower-thanprojected outlays on foreign-financed projects, nonetheless, limited the overall deficit before grants to 10.5 percent of GDP, compared with 11.3 percent envisaged in the program.

\section{The official foreign reserves} target for end-2003 was substantially missed. The gross official reserves of the NBR fell to the equivalent of 5.0 months of import cover at end-2003, compared with $7 \frac{1}{2}$ months targeted in reserves shortfall included the delayed disbursement of US\$62 million in budgetary assistance from the World the program. Factors contributing to the Bank, the European Union and bilateral

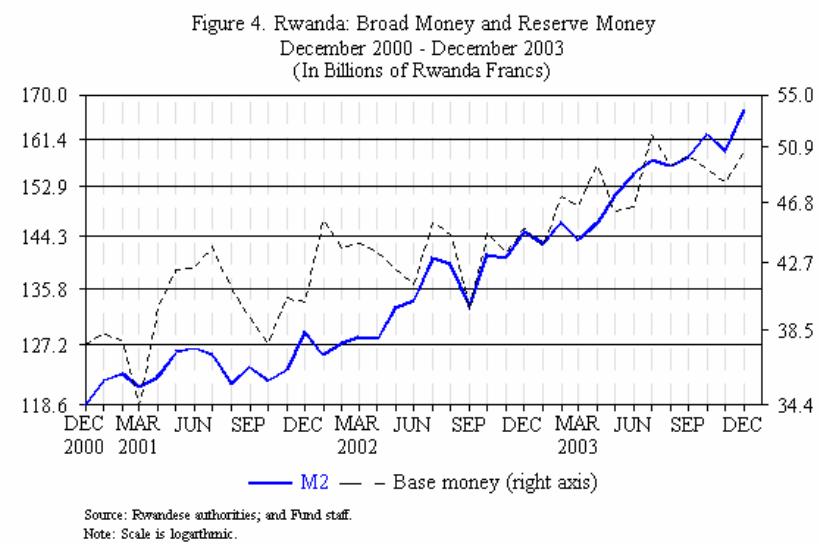

\footnotetext{
${ }^{2}$ The (adjusted) end-June 2003 performance criterion for the domestic fiscal balance was missed by RF 0.9 billion ( 0.1 percent of GDP) as a result of unprogrammed capital transfers to the Banque Commerciale de Rwanda (BCR). The performance criterion for the reduction of domestic arrears at that date was narrowly missed.
} 
partners, and increased National Bank of Rwanda (NBR) foreign exchange sales to reduce liquidity at end-year. As a result of a central bank lending to government, reserve money (evaluated at the program exchange rate) grew by 12 percent during 2003, well above the program's target (Figure 4).

\section{Broad money (at current exchange rates) rose by 15 percent during 2003,} compared with a program target of 8 percent. Banking system credit to government and commercial bank lending for a hotel project contributed to a rapid expansion in the monetary aggregates. ${ }^{3}$ Moreover, the change in the valuation of deposits due to exchange rate depreciation added to broad money growth. Although starting in the fourth quarter the central bank stepped up efforts to tighten liquidity (MEFP paragraph 9), these actions were not fully effective.

\section{Difficulties in the banking sector interfered with the central bank's monetary}

control. Although recoveries on nonperforming loans improved during the first half of 2003, indicators of banking system soundness began to deteriorate during the second half of the year: some banks failed to regularly observe prudential requirements; regulations on net open foreign exchange positions were repeatedly missed; and reserve requirements were occasionally not met.

12. The external current account deteriorated during 2003. Merchandise exports fell marginally, largely due to a drop in coltan and coffee export volumes and weak international market prices for coffee and tea. With imports growing broadly in line with formal sector GDP, the external current account deficit before grants widened to 19.8 percent of GDP in 2003, compared with 18.7 percent in the program.

\section{Rwanda's external debt steadied in 2003, as new borrowing, excluding a new} government loan guarantee, was limited to US\$40 million. However, according to preliminary estimates based on a debt sustainability analysis (DSA) update, Rwanda's net present value (NPV) of debt-to-exports ratio rose to about 300 percent at end-2003, after enhanced HIPC Initiative relief. ${ }^{4}$ The deterioration in this indicator, compared with the decision point projection (190 percent), reflects the impact of the lower reference discount rate and the decline in exports (Box 1). An import related government-guaranteed short-term loan on commercial terms, that formed part of the financing package for the hotel project is slated for liquidation by May 31, 2004 (MEFP, paragraph 13).

\footnotetext{
${ }^{3}$ Following the withdrawal of the majority shareholder in the hotel holding company in mid-2003, the government decided to assure the completion of the hotel project that was underway. The government, as the remaining shareholder, issued guarantees that supported financing through a series of short-term credits.

${ }^{4}$ A full DSA based on loan-by-loan data will be completed ahead of Rwanda's HIPC Initiative completion point consideration.
} 


\section{Box 1. Rwanda: Debt Sustainability}

The staff report for Rwanda's decision point under the HIPC Initiative projected, under its baseline scenario, that Rwanda's net present value of debt to export ratio (NPV) would stand at 193 percent in 2003 and then fall steadily to 149 percent in 2008 and 134 percent by 2019. In the event, a preliminary estimate for 2003 shows Rwanda's NPV to have been 300 percent-well above target. Looking forward, revised projections forecast the NPV to peak at 376 percent in 2005, before beginning a protracted decline that reaches 237 percent in 2019.

Two factors are key to explaining the change in the debt sustainability outlook (see figure below). First, a lower discount rate and exchange rate movements raise the net present value of Rwanda's debt stock by about US\$70 million relative to the decision point document. Second, the initial export projections turned out to be too optimistic: exports of goods and services were expected to reach US\$186 million in 2003 , and then rise to about US $\$ 400$ million by the end of the decade. Exports are now estimated to have totaled US $\$ 126$ million in 2003, and a revised projection for 2009 is, similarly, well below the decision point projection.

Reacting to the weakening of the debt sustainability outlook, the Rwandese authorities have kept new borrowing within the range established in the decision point document: total borrowing amounted to US $\$ 84$ million in 2002 and US\$40 million in 2003. Moreover, borrowing was almost exclusively on highly concessional terms from the World Bank and the African Development Bank.

Looking forward, the authorities face a strong pressure to finance critical social spending and other PRSP-supporting outlays. Nonetheless, unless domestic saving and external grant financing are increased on a sustained basis, government spending may need to be restrained. Against this background, Rwanda needs to move ahead rapidly with the implementation of its export promotion strategy. Simulations show that an additional 12 percent growth of exports over a three-year period starting in 2005 would, other things equal, allow the NPV of debt-to-exports ratio to reach 208 percent bv 2010 .

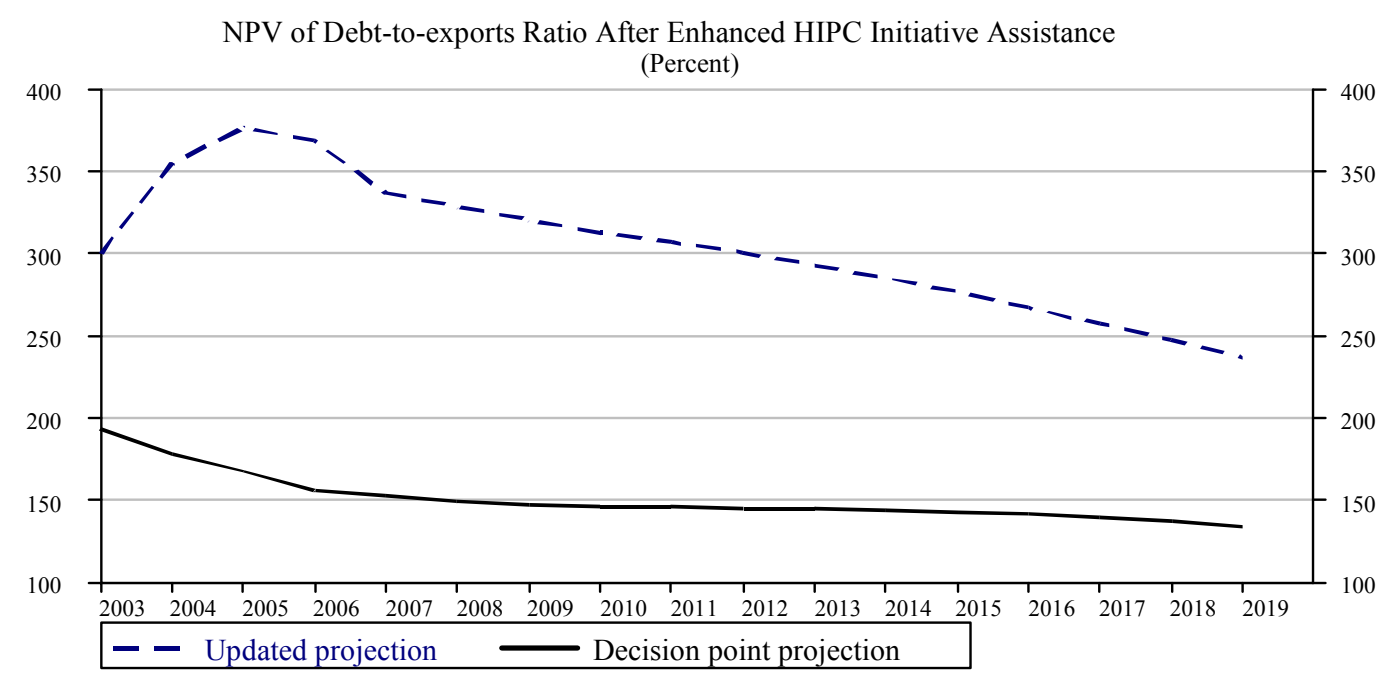


14. Notwithstanding the missed structural performance criteria, substantial progress was made in implementing Rwanda's structural agenda for 2003. With assistance from Fund staff, a new tax law was drafted, incorporating tax provisions that were previously under the authority of the investment promotion agency. Fund staff also assisted in the drafting of a new organic budget law and in finalizing supporting financial instructions for its implementation. The office of the Auditor General (AG), which was further strengthened, audited the 2002 accounts of 41 public entities, including the ministries of Finance and Defense, and issued a report to parliament in March 2004. Moreover, the central bank issued public notices for the closure of government dormant accounts, setting in motion a process that will continue through 2004. In addition, a new chart of accounts was developed for government operations, and an internal audit department was established at the Ministry of Finance.

\section{In the financial sector, steps were taken to improve banking supervision and} alleviate the nonperforming loans problem. However, progress in bank restructuring was slower than targeted (MEFP, paragraphs 16-17). The NBR conducted on-site audits of four commercial banks, established a microfinance sector supervision department, and hired additional supervisors. Nonetheless, the effectiveness of banking supervision continues to be limited by human resource constraints. While steps were taken toward restructuring a mortgage bank and privatizing the Commercial Bank of Rwanda (BCR), neither was fully realized. Following a recapitalization financed by the World Bank, the microfinance institution, Union des Banques Populaire du Rwanda (UBPR), doubled its lending portfolio, with more than a third of the credit growth going to the central government.

\section{POLICY DISCUSSIONS AND REVISED PROGRAM FOR 2004}

\section{A. Poverty Reduction Strategy and Growth}

16. The 2004 program aims at (i) strengthening macroeconomic stability;

(ii) improving economic productivity and external sector performance; and

(iii) accelerating progress toward achieving the government's poverty reduction objectives, including the millennium development goals. Inflation will be substantially reduced, and a return to the target GDP growth trajectory will be supported. In addressing productivity, the authorities intend to update their agricultural strategy, move forward with an export promotion action plan, proceed with the privatization of key sectors, accelerate infrastructure development, and adopt and implement new budget, tax, and investment legislation. Poverty reduction operations include the elimination of fees for primary education, increasing the number of educators, initiating new HIV/AIDS programs, and strengthening public health services. A multi-year labor intensive public works program slated to begin in 2004 will simultaneously address infrastructure and poverty objectives.

17. Under government leadership, and reflecting PRSP priorities, multilateral and bilateral partners have strengthened their cooperation in support of Rwanda's policy program and have substantially increased budgetary financial support. Coordinated 
donor support includes efforts by the World Bank, the EU, the African Development Bank, and the governments of the U.S., U.K., the Netherlands, and Sweden in the areas of agricultural policy, export promotion, decentralization, and public financial management. Moreover, a broad range of partners will collaborate on social policies. Strengthened backing of social policies has been reflected in multiyear commitments from the U.K., the EU and, under a poverty reduction credit currently under preparation, from the World Bank. Reflecting support for these policies, 2004 commitments for external budgetary assistance increased by the equivalent of 4 percentage points of GDP relative to the 2003 outturn (of which 2.2 percentage points correspond to external grant disbursements delayed from 2003). The government is seeking an additional 1.4 percentage points of GDP in grant financing for 2004 to cover various activities, including enhanced export promotion activities and community development. Given the large increase in external assistance, the authorities have committed to ensuring that the use of resources is efficient, and that it is effectively monitored.

18. The macroeconomic framework for 2004 supports the resumption of real economic growth of 6 percent, a reduction of inflation to 5 percent by year's end, and increased spending on poverty reduction. The relaxation of the inflation objective relative to the initial PRGF trajectory (which envisaged a reduction in inflation to 3 percent) takes into account the persistent impact of the exchange rate depreciation in 2003. The import cover of gross official reserves is targeted to fall to 4.6 months, which, while providing broadly adequate cover in the short-term, would not be prudent over the longer term, given Rwanda's susceptibility to external shocks. Therefore, an increase in cover to 5.5 months is targeted by end-2006.

\section{B. Fiscal Policy}

\section{Under the 2004 program, the domestic fiscal deficit, excluding delayed} demobilization outlays and net lending to meet calls on outstanding loan guarantees, is targeted at 5.6 percent of GDP, similar to that of 2003, while the overall deficit would be in balance. This target reflects both the limited scope for new revenue measures in the short run and critical social spending covered by external program grants. Taking into account demobilization operations delayed from 2003 and net lending to meet calls on outstanding government guarantees the domestic fiscal deficit would amount to 7.8 percent of GDP and the overall fiscal deficit (after grants) to 2.2 percent of GDP.,

\section{Domestic revenue is projected to remain stable at 13.5 percent of GDP in 2004 .}

No major tax or nontax revenue policy changes are planned in 2004. A modest fall in customs duties resulting from Rwanda's entrance into the COMESA free trade area on January 1, 2004 is projected to be offset by improved efficiency in tax collection as a result of ongoing reforms at the Rwanda Revenue Authority. Committed external budgetary grants (excluding relief under the HIPC Initiative) are projected at US\$103 million (6.0 percent of GDP) in 2004. As previously noted, the authorities are seeking to raise a further US\$25 million (1.4 percent of GDP) in such funding to cover operations planned for the second half of the year (paragraph 21). In the medium-term, it is expected that improved 
revenue buoyancy will result from a shift in economic activity from the informal to the formal sector.

21. Given the increase in budgetary resources, total expenditure and net lending is programmed to increase to 28.3 percent of GDP in 2004. The authorities explained that the budget includes substantial funding increases for the education, health, and road sectors, and stepped-up financing for growth enhancing activities envisaged under the PRSP. In addition, expenditures include net lending to cover costs incurred during 2003 on the above noted hotel project, and to finance the purchase of electric generators to address a droughtrelated drop in hydroelectric output. In response to staff concerns regarding the risks associated with their less restrained fiscal program for 2004, the authorities revised the expenditure profile. Specifically, part of nonpriority spending was rephased to the second half of 2004 to facilitate fiscal restraint if additional grant financing is not forthcoming.

\section{The deficit will be fully covered by external grants and external program} borrowing (US $\$ 45$ million) under a previously approved World Bank program credit and an African Development Bank credit.

\section{Monetary Policy}

23. The monetary program seeks to limit inflation to 5 percent at end-2004 On this basis, and taking into account the projected growth of the monetized sector of the economy, the target for broad money growth has been set at 11 percent. Based on the fiscal program described above, the monetary program would provide room for increasing banking system credit to the private sector by 11 percent, in line with GDP growth. The authorities will maintain a managed float of the foreign exchange system. ${ }^{5}$

24. Efforts to strengthen the financial sector will continue in 2004 (MEFP, paragraphs 47-48). With due diligence inspections completed early in 2004, the privatization of the Commercial Bank of Rwanda is targeted by end-July 2004. Given the departures from prudential regulations during 2003, the central bank will reach agreements with banks by end-June 2004 on action plans to bring all commercial banks back into regular compliance. Banking supervision will be reinforced, as an enhanced supervisory regime will continue for two banks, and on-site audits are planned for five banks. In addition, a joint Fund-World Bank financial sector assessment is scheduled for the fourth quarter of 2004.

\section{External Sector Policies}

25. The external current account deficit (after grants) is projected to narrow by 1.5 percentage points of GDP, to 6.9 percent in 2004 (MEFP, paragraph 33). Early efforts

\footnotetext{
${ }^{5}$ Central bank intervention in the foreign exchange market will be limited to supplying a predetermined amount of foreign exchange in the weekly auctions and to smoothing market disturbances that are unrelated to underlying market fundamentals.
} 
to strengthen export performance, including targeted activities in the coffee and tea sectors, are projected to yield solid returns during 2004, starting from a limited base. Merchandise imports are projected to increase by 16 percent in U.S. dollar terms, mainly reflecting outlays for the growing development budget and intermediate goods, including fertilizer. In the medium term, and subject to vigorous implementation, the export promotion strategy is expected to substantially strengthen Rwanda's export performance. While the authorities expect the strategy to substantially strengthen export performance in the near term, Fund staff noted that the realization of accelerated export growth is not certain, as elements of the strategy have yet to be finalized. Given this, staff urged the authorities to take the necessary action, in cooperation with supporting partners, to assure the strategy's success.

\section{Projected donor disbursements and debt reschedulings would fully finance the} 2004 program. Balance of payments support is projected at US\$174 million, mainly from the World Bank, the UK, the EU, and some smaller bilateral donors. HIPC Interim Assistance and bilateral debt relief are projected at US\$41 million. ${ }^{6}$

\section{E. Structural Reforms}

27. The structural program for 2004 focuses on improving economic productivity, further strengthening the legal framework and public finances, and continuing financial sector reforms. The authorities indicated that this agenda seeks to address the highest priority structural elements of the PRSP's economic agenda (Box 2). In addressing the weak external sector performance, in particular, the sale of tea factories, the introduction of a strengthened investment code, and the implementation of the export promotion action plan are projected to result in prompt and tangible returns (MEFP, paragraphs 34-35). The authorities have also committed to promptly selling the government's stake in the recently completed hotel project.

28. The enactment of the organic budget law, income tax law, and customs and public procurement laws targets the creation of a modern, efficient and transparent financial administration (MEFP, paragraph 40). The agenda for public administration reform will continue with the strengthening of budgeting, internal accounting and decentralization, including through the extension the government's computerized accounting system to the provincial level. Work will begin on the introduction of a treasury single account and key improvements to the budget document (MEFP, paragraphs 43-44).

\footnotetext{
${ }^{6}$ Firm commitments to provide relief under the HIPC Initiative, from some non-Paris Club creditors including the Abu Dhabi Fund for Development, and Libya, have not yet been received.
} 


\section{Box 2: Structural Conditionality}

\section{Coverage of structural conditionality in the 2004 program}

Three prior actions have been set for the completion of the second and third reviews, and one structural performance criterion and three structural benchmarks for the fourth review. The prior actions cover public procurement legislation, a strengthening of NBR statistics, and NBR regulations on the net open foreign exchange position of commercial banks. The submission of a revised 2004 budget to parliament, a structural performance criterion for June 2004, will modify the budget in line with the 2004 program. The benchmarks on monthly reconciliation statements of government accounts and inclusion of consolidated government accounts in the 2005 budget seek to improve fiscal transparency. The benchmark on approval of the export promotion plan reflects the authorities' strengthened commitment to addressing Rwanda's external sustainability, while the benchmark on external audit of the 2003 NBR accounts follows the recommendations of the 2003 Safeguards mission. Finally, the benchmark for the NBR to set and enforce dates for bringing banks into compliance with prudential regulations will strengthen the soundness of the banking system and improve the effectiveness of monetary policy instruments.

\section{Status of previously established structural conditionality}

The 2003 program established structural performance criteria, benchmarks and prior actions scheduled for June-September 2003 (MEFP, Table 2). For the second review, the authorities met the two performance criteria that were set. However, only one of the benchmarks for the second review was observed. Nonetheless, substantial progress was made: the report on the 2002 development budget and list of overdue government obligations to be cleared in 2003 were issued by September 2003; and the process for the sale of BCR was set firmly in motion. The NBR is finalizing the written procedures for assuring consistency between data submitted to the IMF and commercial bank submission. The completion of these procedures by end-May, 2004, has been set as a prior action for this review. The NBR met a September 2003 benchmark by issuing public notifications required for the closure of dormant government accounts and readying an action plan for closure of these accounts. AFRITAC East staff are scheduled, during 2004, to provide technical assistance that will lead to monthly reporting of district financial operations, by end-December 2004. While a new organic budget law drafted with assistance from Fund staff is slated for parliamentary submission by end-June 2004, the end-September 2003 target date was missed.

Three performance criteria and one benchmark were set for the third review. The benchmark for operationalizing internal audit procedures within the NBR was observed, a revised law incorporating tax incentives into the income tax has been readied and is slated for submission to parliament by end-June 2004 , and a revised investment code is in preparation. While financial instructions for the implementation of the new organic budget law have been readied, their issuance has been delayed to coincide with approval of the new law.

\section{Structural measures covered by World Bank lending and conditionality}

The World Bank's lending programs are aimed at improvements in the following areas:

- Public finances: medium term expenditure framework, poverty monitoring, capacity building at Ministry of Finance and Auditor General's Office;

- Financial sector: Improved loan recovery, arbitration center, credit unions (UBPR), housing finance (CHR), privatization of the Bank of Kigali;

- Governance and the business environment: creation of commercial courts, National Tender Board, strengthening the investment promotion authority (RIPA);

- Regulations and privatization, including the transfer to the private sector of Rwandatel, Electrogaz, and coffee and tea state factories.

Human resources: adoption of sector-wide approaches, immunization, health education, improved access to health services. 


\section{F. Governance and Safeguards}

29. Rwanda has made significant efforts toward the establishment of an open and transparent public financial administration. Reforms are underway to address issues identified in the Auditor General's report to parliament, including the required strengthening of public procurement procedures, and plans have been developed to continue with the recruitment and training of accountants and financial controllers in line ministries. The cabinet is also slated to approve a strengthened public tender code by end-May 2004 and, following the adoption of legislation currently under consideration by parliament, the AG's office will produce annual audit reports of public entities as part of the annual audit starting in 2004.

30. On the basis of a Fund safeguards assessment in early 2003, the National Bank of Rwanda developed an action plan to strengthen its autonomy, external and internal audit mechanism, the legal structure, and financial reporting and internal controls. Since then, some of the key recommendations of the safeguards assessment have been implemented. In particular, a regular, annual external audit process has been established with the audited financial statements to be published within six months of the financial year. Moreover, the NBR agreed to limit the 2004 distributable profit to realized gains. Furthermore, the Internal Audit Department is being operationalized, with the Internal Audit Charter approved and the audit risk assessment of all NBR operations completed.

\section{Development and enforcement of anti-money laundering legislation is} progressing slowly. An initial draft of the anti-money laundering law was deemed unsatisfactory by the government, and the NBR has requested Fund technical assistance to support its next steps in this area.

\section{G. Statistical Issues}

32. Rwanda's macroeconomic data are adequate for program monitoring purposes. Rwanda has participated in the Funds' General Data Dissemination System since endOctober 2003. Nevertheless, significant weaknesses remain. In particular, reconciliation of fiscal and monetary data is constrained by the large number of government accounts, the current consumer price index is outdated, the quality and timeliness of output data is inadequate, and only limited information is available on monthly and quarterly capital account transactions. The authorities are taking steps to improve the situation. The introduction of a single treasury account at the NBR is envisaged for 2005, a new consumer price index is being tested and will be published in June 2004, and the coverage and quality of balance of payments data is being improved with Fund technical assistance. Furthermore, a new independent Statistics Office is being established with financial assistance from development partners.

\section{H. Program Monitoring}

33. The fourth review will assess performance with respect to the quantitative performance criteria for end-June 2004. In addition, it will assess performance with 
respect to the structural performance criteria for end-June 2004 and three structural benchmarks through end-September $2004 .^{7}$ The attached technical memorandum of understanding (Appendix I, Attachment II) details the design of the program, adjustment mechanisms and terminology.

\section{Staff Appraisal}

34. Increased external assistance inflows in $\mathbf{2 0 0 4}$ will provide Rwanda with an opportunity to accelerate progress on its PRSP agenda and towards the achievement of the Millennium Development Goals. Given the recent progress made towards achieving peace in the region and consolidating the political process, the efficient use of these resources would establish a credible basis for continued and, possibly, increased aid commitments.

35. The more expansionary fiscal stance for 2004, which is supported by increased external assistance, entails several challenges that need to be addressed if the poverty and growth objectives are to be achieved and sustained. In particular, the issues of external debt sustainability, increased relative prices for nontradables, and medium and longrun fiscal sustainability must be tackled if the authorities' strategy is to succeed. The 2004 program reflects discussions on the appropriate balance in each of these areas, along with supporting policy commitments.

36. While program implementation in 2003 was disappointing and economic performance in $\mathbf{2 0 0 3}$ fell well short of the program objectives, the implementation of the 2004 program will be facilitated by the improved political situation and the more stable regional environment. With a new executive administration and legislature in place, an expanded resource envelope, and good rainfall in the second agricultural season, the authorities will benefit from a far more favorable environment for the achievement of the 2004 program targets. The success of Rwanda's poverty reduction strategy hinges on enhancing productivity to promote strong growth in formal sector activity and, with this, increasing nondebt financial inflows and domestic saving.

37. The increase in external budgetary support programmed for 2004 will provide the government with substantial additional resources to finance its operations. Over the medium-term, the complementary strengthening of the domestic resource base will benefit from the prospective revision of tax and investment laws and tax administration regulations. The initial steps taken in this direction are commendable. In moving forward, the authorities will need to ensure that the integrity of the revenue base is maintained and that new initiatives do not detract from that effort.

38. The budgeted increase in government spending for 2004 balances funding for social programs, including free primary schooling and extended health services, against

\footnotetext{
${ }^{7}$ Tables 1 and 2 in the attached MEFP set out the complete structure of conditionality, and Box 2 explains the macroeconomic relevance of the structural measures included in the program.
} 
operations such as road development and export promotion that will address supply constraints in the economy. While it would have been preferable for the decisions taken on hotel financing in 2003 to have been made within the PRSP and budget frameworks, the regularization of these expenditures as part of the revised 2004 budget and the planned prompt sale of the hotel are important steps in the right direction. In this context, the decision to rephase part of nonpriority expenditure to the second half of 2004, in order to assure that these resources are used effectively, is appropriate. As the outlook for raising additional external grant financing is uncertain, however, the authorities should be prepared to postpone or reduce spending as needed.

\section{The solid policy and administrative frameworks that underpin health and} education operations, developed in concert with Rwanda's supporting partners, will help ensure that the additional resources are well used. The authorities should attach a high priority to efforts supported by the EU, World Bank and other partners to develop strong frameworks for the remaining economic sectors, including agriculture, energy, and exports. In addition, a decisive implementation of actions to strengthen the financial administration of provincial and local government units will be critical in the period ahead, given the progressive decentralization of government.

40. Limiting liquidity growth, given the substantial increase in government spending budgeted for 2004, poses an extraordinary challenge for the monetary authorities. In order to constrain the expansion of the monetary aggregates, the central bank must assure that the foreign exchange auction operates efficiently and that commercial banks are brought quickly into compliance with the regulatory framework, including reserve requirements. Beyond this, the financial sector assessment, to be conducted later in 2004, is likely to provide additional recommendations for strengthening Rwanda's financial sector and, thus, the effectiveness of monetary policy.

41. The increased external assistance supporting Rwanda's efforts to reduce poverty will, in the near term, be accompanied by a deterioration in the trade balance. Over the medium-term, under Rwanda's poverty reduction strategy, this deterioration is expected to gradually reverse, as supply side constraints are addressed and export performance improves. While projected increases in commodity export prices and good weather will contribute to ending the slide in merchandise exports during 2004, the authorities need to begin implementing effectively their export promotion strategy.

42. Serious concerns remain regarding Rwanda's external debt sustainability. Given Rwanda's elevated NPV of debt-to-exports ratio at end-2003, new external borrowing should be strictly limited Full implementation of the programs to address Rwanda's social needs, along with the investment required to sustain high growth rates, will entail elevated fiscal deficits over the medium-term. Given this, the donor community will need to focus on providing external grant assistance to support the achievement of Rwanda's poverty reduction and growth objectives. 
43. Although some progress has been made in implementing recommendations by the Auditor General in 2002, the findings of the audits point to significant weaknesses in public resource management. In particular, continued weaknesses of line ministries in the documentation of their use of allocations, in part related to dearth of qualified accountants, needs to be promptly addressed. The government's plans to address problems in the operation of the public procurement process are of critical importance and should be implemented expeditiously and decisively.

44. In sum, despite the slippages in macroeconomic performance that took place in 2003, Rwanda, having faced extraordinary challenges in the post-genocide period, has set in place a sound institutional and policy foundation for its economy. The 2004 program builds on this framework. The targeted reduction in inflation and GDP growth are realistic and appropriate. Looking forward, strong policy implementation will be critical, in order to secure continued donor assistance and promote private sector investment. Available data, through end-April 2004, indicate that a good start has been made towards these objectives.

45. Based on the above, the staff recommends that the requested waivers for the nonobservance of the performance criteria on domestic fiscal balance, the net accumulation of domestic arrears, and new nonconcessional external debt at end-June 2003, and the domestic fiscal balance, net accumulation of domestic arrears, net credit to government by the banking system, reserve money, net foreign assets of the National Bank of Rwanda (NBR), and new nonconcessional external debt at end-December 2003, and the nonobservance of the structural performance criteria on the submission of a revised investment code, the issuance of financial instructions, and the incorporation of tax incentives into the structure of the tax law be granted, and that the second and third reviews under the PRGF arrangement be completed.

46. Satisfactory assurances of HIPC assistance were provided by creditors at the time of the decision point. These assurances remain in place. Staff also recommends the extension of provision of additional interim HIPC Initiative assistance for the period June 9, 2004 to June 8, 2005. 
Table 1: Rwanda: Selected Economic and Financial Indicators, 2001-06

\begin{tabular}{|c|c|c|c|c|c|c|c|c|c|c|}
\hline & \multirow[t]{2}{*}{2001} & \multicolumn{2}{|c|}{2002} & \multicolumn{3}{|c|}{2003} & \multicolumn{2}{|c|}{2004} & \multirow{2}{*}{$\frac{2005}{\text { Proj. }}$} & \multirow[t]{2}{*}{2006} \\
\hline & & PRGF 1/ & $\overline{\text { Act. }}$ & PRGF 1/ & Prog. 1/ & $\overline{\text { Est. }}$ & Prog. 1/ & $\begin{array}{c}\text { Rev. } \\
\text { Prog. }\end{array}$ & & \\
\hline & \multicolumn{10}{|c|}{ (Annual percentage changes, unless otherwise indicated) } \\
\hline \multicolumn{11}{|l|}{ Output and prices } \\
\hline Real GDP growth & 6.7 & 7.3 & 9.4 & 6.1 & 3.2 & 0.9 & 6.0 & 6.0 & 6.0 & 6.0 \\
\hline GDP deflator & 0.2 & 1.9 & 0.0 & 3.5 & 4.9 & 8.7 & 2.7 & 5.0 & 4.0 & 4.0 \\
\hline Consumer prices (period average) & 3.4 & 2.0 & 2.0 & 3.0 & 4.7 & 7.4 & 3.0 & 6.9 & 4.0 & 4.0 \\
\hline Consumer prices (end of period) & -0.2 & 3.0 & 6.2 & 3.0 & 3.0 & 7.7 & 3.0 & 5.0 & 4.0 & 4.0 \\
\hline \multicolumn{11}{|l|}{ External sector } \\
\hline Export, f.o.b (in U.S. dollars) & 4.2 & -16.9 & -28.1 & 12.1 & 6.6 & -6.3 & 10.6 & 8.0 & 17.0 & 11.6 \\
\hline Imports, f.o.b (in U.S. dollars) & -0.8 & 0.5 & -1.3 & 3.2 & 8.5 & 4.0 & 2.8 & 16.0 & 7.1 & 6.2 \\
\hline Export volume & 36.8 & -13.8 & -17.1 & 6.5 & 1.2 & -13.2 & 4.1 & 2.6 & 12.3 & 10.7 \\
\hline Import volume & 2.3 & -0.4 & -6.3 & 2.9 & 3.4 & 0.6 & 3.8 & 11.8 & 7.5 & 5.8 \\
\hline Terms of trade (deterioration -) & -21.5 & -4.5 & -17.6 & 5.1 & 0.4 & 4.4 & 7.2 & 1.4 & 4.6 & 0.5 \\
\hline \multicolumn{11}{|l|}{ Government finance } \\
\hline Revenue & 25.5 & 16.9 & 16.9 & 14.0 & 18.5 & 21.4 & 10.7 & 10.8 & 9.9 & 9.6 \\
\hline Total expenditure and net lending & 20.0 & 15.5 & 11.3 & 9.6 & 15.1 & 23.8 & -3.7 & 30.8 & 0.8 & 5.2 \\
\hline Current expenditure & 20.4 & 16.6 & 15.2 & 7.3 & 20.7 & 31.5 & -8.3 & 9.9 & 1.4 & 1.9 \\
\hline \multicolumn{11}{|l|}{ Money and credit 2/ } \\
\hline Net domestic assets 3 / & -2.6 & 1.3 & -6.1 & $\ldots$ & -17.0 & 14.8 & $\ldots$ & 11.0 & $\ldots$ & $\ldots$ \\
\hline Domestic credit 3/ & 0.3 & 0.7 & -4.0 & $\ldots$ & -15.5 & 19.7 & $\ldots$ & 9.7 & $\ldots$ & $\ldots$ \\
\hline Government 3/ & -5.1 & -7.0 & -12.4 & $\ldots$ & -20.2 & 10.7 & $\ldots$ & 3.2 & $\ldots$ & $\ldots$ \\
\hline Economy $3 /$ & 5.4 & 7.6 & 8.5 & $\ldots$ & 4.7 & 9.0 & $\ldots$ & 6.5 & $\ldots$ & $\ldots$ \\
\hline Money and quasi money (M2) & 9.2 & 6.9 & 11.4 & $\ldots$ & 8.0 & 15.2 & $\ldots$ & 11.0 & $\ldots$ & $\ldots$ \\
\hline Reserve money & 6.6 & 6.3 & 11.9 & $\ldots$ & 7.1 & 12.2 & $\ldots$ & 11.6 & $\ldots$ & $\ldots$ \\
\hline Velocity (GDP/M2; end of period) & 6.1 & 5.9 & 6.0 & $\ldots$ & 5.7 & 5.4 & $\ldots$ & 5.4 & $\ldots$ & $\ldots$ \\
\hline \multicolumn{11}{|c|}{ (In percent of GDP, unless otherwise indicated) } \\
\hline National income accounts & & & & & & & & & & \\
\hline National savings (excluding official transfers) & 2.5 & 1.8 & 0.3 & 3.5 & 1.2 & -1.4 & 2.6 & -0.1 & 1.5 & 2.5 \\
\hline Of which: private (including public enterprises) & 5.4 & 4.9 & 4.5 & 5.8 & 4.8 & 3.5 & 3.2 & 6.1 & 4.5 & 4.4 \\
\hline Gross investment & 18.4 & 18.8 & 16.9 & 19.3 & 19.9 & 18.4 & 20.0 & 21.3 & 22.3 & 22.7 \\
\hline Of which: private (including public enterprises) & 11.8 & 12.0 & 12.0 & 12.2 & 12.2 & 12.8 & 12.4 & 12.6 & 12.8 & 13.0 \\
\hline \multicolumn{11}{|l|}{ Government finance } \\
\hline Total revenue & 11.4 & 12.2 & 12.2 & 12.7 & 13.4 & 13.5 & 13.6 & 13.5 & 13.5 & 13.6 \\
\hline Total expenditure and net lending & 21.0 & 22.1 & 21.3 & 22.1 & 24.7 & 24.1 & 21.9 & 28.3 & 26.1 & 25.1 \\
\hline Capital expenditure & 6.6 & 7.0 & 4.9 & 7.3 & 7.7 & 5.6 & 7.7 & 8.7 & 9.5 & 9.7 \\
\hline Current expenditure & 14.2 & 15.2 & 15.0 & 14.8 & 16.7 & 18.0 & 14.1 & 17.8 & 16.5 & 15.4 \\
\hline Primary fiscal balance 4 / & -0.1 & -0.2 & -1.6 & 0.7 & 0.8 & -0.3 & 1.4 & -2.6 & -2.6 & -1.7 \\
\hline Domestic fiscal balance 5/ & -2.7 & -3.3 & -4.2 & -2.8 & -4.4 & -5.5 & -1.1 & -7.8 & -5.2 & -4.0 \\
\hline \multicolumn{11}{|l|}{ Overall balance (payment order) } \\
\hline After grants & -1.3 & -1.1 & -1.9 & -0.6 & 0.9 & -2.5 & 0.7 & -2.2 & -0.7 & -1.0 \\
\hline Before grants & -9.5 & -9.9 & -9.1 & -9.4 & -11.3 & -10.5 & -8.2 & -14.8 & -12.5 & -11.5 \\
\hline \multicolumn{11}{|l|}{ External sector } \\
\hline External current account balance & & & & & & & & & & \\
\hline Including official transfers $6 /$ & -5.9 & -11.3 & -6.7 & -11.5 & -11.3 & -8.4 & -12.3 & -6.9 & -8.2 & -9.4 \\
\hline Excluding official transfers & -15.9 & -17.0 & -16.6 & -15.7 & -18.7 & -19.8 & -17.4 & -21.4 & -20.8 & -20.1 \\
\hline External debt (end of period) $7 /$ & 78.4 & 73.2 & 80.9 & 66.8 & 86.1 & 85.1 & 82.2 & 86.6 & 85.7 & 78.5 \\
\hline Net present value of external debt & & & & & & & & & & \\
\hline (in percent of exports of goods and services) 8 / & 236.2 & 190.3 & 263.0 & 203.5 & 222.2 & 299.9 & 242.1 & 354.5 & 376.4 & 368.6 \\
\hline Scheduled debt-service ratio after HIPC Initiative debt relief & & & & & & & & & & \\
\hline (in percent of exports of goods and services) & 9.3 & 15.4 & 13.8 & 9.1 & 9.1 & 10.3 & 10.0 & 9.6 & 12.7 & 10.5 \\
\hline Gross reserves (in months of imports of goods and services) & 6.0 & 5.9 & 6.3 & 6.2 & 7.4 & 5.0 & 6.9 & 4.6 & 5.2 & 5.5 \\
\hline & & & & & In million & of U.S. do & rs, unless o & herwise in & ated) & \\
\hline External debt (end of period) 7/ & $1,336.1$ & $1,295.1$ & $1,401.0$ & $1,268.4$ & $1,471.4$ & $1,432.8$ & $1,493.0$ & $1,485.5$ & $1,556.2$ & $1,510.0$ \\
\hline Gross official reserves & 212.1 & 223.6 & 243.6 & 236.6 & 299.1 & 214.2 & 282.5 & 208.6 & 251.5 & 276.2 \\
\hline Memorandum item: & & & & & & & & & & \\
\hline Nominal GDP (in billions of Rwanda francs) & 754.3 & 825.2 & 825.0 & 906.4 & 893.4 & 905.3 & 971.9 & $1,004.9$ & $1,100.5$ & $1,201.3$ \\
\hline
\end{tabular}

Sources: Rwandese authorities; and staff estimates and projections.

1/ 2002-03 PRGF: see IMF Country Report No. 02/204; and 2003-04 prog.: see IMF Country Report No. 03/192.

2/ Numbers up to 2003 are based on current exchange rates, while 2004-06 numbers are based on the program exchange rate of RF 580.3/U.S. dollar.

3/ As a percent of the beginning-of-period stock of broad money.

4/ Revenue excluding grants; minus current expenditure except interest due and exceptional expenditure;

minus domestically financed capital expenditure.

5/ Revenue excluding grants; minus current expenditure (excluding external interest), minus domestically-financed capital expenditure and net lending.

6/ In the "Prog." and "PRGF" columns for 2002 and 2003, official transfers exclude expected but not yet disbursed budgetary assistance.

$7 /$ After rescheduling, including arrears and new debt (the latter includes assumed project and budgetary disbursements for the period 2004-06). The data under the "PRGF"

and the "Prog."/"Est." Columns are not comparable since they are based on a different treatment of projected loan disbursements. Data are preliminary and do not

reflect changes in exchange rates.

8/ Based on assumptions about expected new borrowing. For illustrative purposes, the numbers are shown as if HIPC Initiative assistance had been delivered unconditionally

as of 1999 (data available at the time of the decision point). The exports denominator is calculated using a three-year backward-looking average. Data are preliminary,

pending a full debt sustainability analysis

\section{CInternational Monetary Fund. Not for Redistribution}


Table 2. Rwanda: Operations of the Central Government, 2001-06

\begin{tabular}{|c|c|c|c|c|c|c|c|c|c|c|c|c|c|c|}
\hline \multirow[t]{3}{*}{2001} & \multirow[t]{3}{*}{2002} & \multicolumn{6}{|c|}{2003} & \multicolumn{5}{|c|}{2004} & \multirow{3}{*}{$\begin{array}{l}2005 \\
\text { Rev. } \\
\text { Proj. }\end{array}$} & \multirow{3}{*}{$\begin{array}{l}2006 \\
\text { Rev } \\
\text { Proj. }\end{array}$} \\
\hline & & \multicolumn{2}{|c|}{ June } & \multicolumn{2}{|c|}{ Sep. } & \multicolumn{2}{|c|}{ Dec. } & \multirow[t]{2}{*}{ Mar. } & \multirow{2}{*}{$\begin{array}{l}\text { June } \\
\text { Rev. } \\
\text { Prog. }\end{array}$} & \multirow{2}{*}{$\begin{array}{l}\text { Sep. } \\
\text { Rev. } \\
\text { Prog. }\end{array}$} & \multicolumn{2}{|c|}{ Dec. } & & \\
\hline & & Prog. 1/ & Prel. & Prog. 1/ & Prel. & Prog. 1/ & Est. & & & & Prog. 1/ & $\begin{array}{r}\text { Rev. } \\
\text { Prog. }\end{array}$ & & \\
\hline
\end{tabular}

\begin{tabular}{|c|c|c|c|c|c|c|c|c|c|c|c|c|c|c|c|}
\hline \multirow[b]{2}{*}{ Revenue and grants } & \multicolumn{15}{|c|}{ (In billions of Rwanda francs) } \\
\hline & 148.2 & 160.3 & 101.2 & 93.2 & 159.3 & 134.4 & 228.5 & 195.5 & 60.4 & 125.7 & 190.6 & 218.7 & 262.4 & 278.9 & 290.4 \\
\hline Total revenue & 86.2 & 101.2 & 57.6 & 59.9 & 88.4 & 90.3 & 119.4 & 122.3 & 31.3 & 67.4 & 100.2 & 132.2 & 135.6 & 149.0 & 163.3 \\
\hline Tax revenue & 79.5 & 94.6 & 53.4 & 55.4 & 82.8 & 83.9 & 112.4 & 114.6 & 28.1 & 61.1 & 92.5 & 125.1 & 126.3 & 139.7 & 153.1 \\
\hline Direct taxes & 24.5 & 30.5 & 15.3 & 17.6 & 24.0 & 25.4 & 32.0 & 35.1 & 8.6 & 19.7 & 28.5 & 36.0 & 37.8 & 40.5 & 44.0 \\
\hline Taxes on goods and services & 41.0 & 47.4 & 27.5 & 27.4 & 42.6 & 42.4 & $\begin{array}{l}52.0 \\
58.2\end{array}$ & 57.5 & $\begin{array}{l}8.0 \\
14.7\end{array}$ & 30.6 & 47.3 & 64.0 & $\begin{array}{l}63.8 \\
63.8\end{array}$ & 71.2 & 78.3 \\
\hline Taxes on international trade & 14.0 & 16.7 & 10.6 & 10.4 & 16.2 & 16.1 & 22.3 & 22.1 & 4.8 & 10.8 & 16.8 & 25.0 & 24.7 & 28.1 & 30.8 \\
\hline Nontax revenue & 6.7 & 6.6 & 4.2 & 4.6 & 5.6 & 6.4 & 7.0 & 7.7 & 3.2 & 6.3 & 7.7 & 7.1 & 9.3 & 9.3 & 10.2 \\
\hline Grants & 62.0 & 59.1 & 43.6 & 33.3 & 71.0 & 44.1 & 109.1 & 73.1 & 29.1 & 58.3 & 90.4 & 86.5 & 126.9 & 129.9 & 127.1 \\
\hline Budgetary grants & 33.9 & 39.3 & 25.9 & 22.2 & 44.4 & 27.5 & 73.6 & 51.0 & 20.4 & 40.9 & 64.3 & 46.1 & 92.1 & 83.4 & 73.7 \\
\hline Of which: HIPC Initiative assistance & 10.6 & 12.2 & 5.1 & 5.8 & 9.0 & 9.3 & 11.6 & 11.5 & 2.8 & 7.2 & 10.9 & 15.8 & 15.9 & 19.3 & 0.0 \\
\hline Capital grants & 28.1 & 19.8 & 17.7 & 11.1 & 26.6 & 16.6 & 35.4 & 22.1 & 8.7 & 17.4 & 26.1 & 40.4 & 34.8 & 46.5 & 53.4 \\
\hline Total expenditure and net lending & 158.1 & 175.9 & 112.0 & 100.6 & 166.1 & 160.9 & 220.5 & 217.8 & 58.6 & 135.6 & 206.9 & 212.4 & 284.7 & 287.0 & 302.0 \\
\hline Current expenditure & 107.4 & 123.7 & 75.5 & 73.4 & 112.3 & 118.5 & 149.3 & 162.7 & 39.4 & 81.8 & 127.2 & 136.9 & 178.8 & 181.3 & 184.8 \\
\hline Of which: priority & 40.1 & 50.4 & 30.3 & 30.6 & 42.6 & 44.8 & 56.2 & 59.1 & 13.9 & 35.5 & 59.0 & $\begin{array}{r}100.9 \\
64.0\end{array}$ & $\begin{array}{r}82.0 \\
82.7\end{array}$ & 93.5 & 105.6 \\
\hline Wages and salaries & 38.9 & 40.6 & 21.0 & 21.8 & 31.6 & 32.8 & 42.1 & 44.0 & 12.0 & 24.0 & 36.0 & 43.8 & 48.0 & 51.3 & 54.5 \\
\hline Civil & 22.8 & 24.1 & 13.9 & 13.8 & 20.8 & 21.0 & 27.8 & 28.3 & 7.9 & 15.7 & 23.6 & 30.2 & 31.4 & 34.7 & 37.4 \\
\hline Defense & 16.2 & 16.5 & 7.2 & $\begin{array}{r}7.80 \\
7.9\end{array}$ & 10.8 & 11.8 & 14.3 & $\begin{array}{l}20.3 \\
15.7\end{array}$ & 4.1 & 8.3 & 12.4 & 13.6 & $\begin{array}{l}1.4 \\
16.5\end{array}$ & 16.6 & 17.1 \\
\hline Purchases of goods and services & 29.4 & 35.2 & 21.1 & 22.1 & 26.6 & 32.1 & 33.6 & 45.8 & 11.0 & 21.5 & 36.6 & 37.1 & 52.6 & 53.2 & 53.4 \\
\hline Civil & 20.4 & 27.3 & 15.1 & 16.6 & 19.4 & 25.0 & 24.9 & 37.1 & 9.0 & 17.0 & 30.1 & 27.7 & 43.5 & 43.5 & 43.1 \\
\hline Of which: contigent 2 & 0.0 & 0.0 & 0.0 & 0.0 & 0.0 & 0.0 & 0.0 & 0.0 & 0.0 & 0.0 & 2.9 & 0.0 & 6.4 & 0.0 & 0.0 \\
\hline Defense & 9.0 & 7.8 & 6.0 & 5.5 & 7.2 & 7.1 & 8.7 & 8.6 & 2.0 & 4.5 & 6.5 & 9.4 & 9.1 & 9.7 & 10.3 \\
\hline Interest payments & 6.2 & 8.3 & 4.3 & 5.2 & 6.5 & 7.1 & 9.5 & 10.5 & 2.3 & 7.0 & 8.9 & 9.8 & 14.7 & 15.1 & 15.1 \\
\hline Domestic debt (due) & 1.3 & 2.1 & 1.1 & 1.5 & 1.6 & 1.8 & 2.1 & 3.17 & 0.42 & 2.56 & 3.00 & 2.3 & 6.6 & 6.5 & 6.4 \\
\hline External debt (due) & 5.0 & 6.2 & 3.2 & 3.7 & 4.9 & 5.3 & 7.3 & 7.32 & 1.90 & 4.46 & 5.91 & 7.5 & 8.1 & 8.7 & 8.7 \\
\hline Transfers & 15.1 & 20.4 & 11.5 & 10.6 & 16.8 & 16.2 & 22.3 & 22.2 & 7.0 & 16.3 & 26.1 & 25.1 & 36.2 & 40.1 & 41.8 \\
\hline Exceptional expenditure & 17.7 & 19.2 & 17.6 & 13.7 & 30.7 & 30.4 & 41.8 & 40.2 & 7.1 & 13.0 & 19.6 & 21.1 & $\begin{array}{l}20.2 \\
27.3\end{array}$ & 21.5 & 20.0 \\
\hline Of which: demobilization/reintegration/reeducation & 3.4 & 3.4 & 7.7 & 3.8 & 10.6 & 4.6 & 13.7 & 7.3 & 3.5 & 7.5 & 9.9 & 5.2 & 12.6 & 2.4 & 0.0 \\
\hline one-off: referendum / elections / health & 0.0 & 0.0 & 3.2 & 2.2 & 5.6 & 13.0 & 9.3 & 14.8 & 0.081 & 0.2 & 0.2 & 0.0 & 0.3 & 0.0 & 0.0 \\
\hline Capital expenditure & 50.0 & 40.7 & 35.3 & 26.2 & 52.1 & 38.9 & 69.0 & 51.1 & 17.9 & 37.5 & 61.9 & 74.5 & 87.2 & 104.7 & 116.2 \\
\hline Of which: priority & 0.0 & 1.0 & 1.2 & 0.5 & 2.4 & 2.0 & 4.0 & 3.0 & 1.0 & 2.0 & 3.5 & 5.5 & 5.5 & 7.0 & 8.0 \\
\hline Domestic & 3.5 & 6.9 & 8.0 & 7.3 & $\begin{array}{r}2.4 \\
11.1\end{array}$ & 10.5 & 14.3 & $\begin{array}{r}5.0 \\
13.2\end{array}$ & 2.3 & 6.3 & 15.2 & 12.2 & 24.9 & 33.0 & 33.9 \\
\hline Of which: Common Development Fund (CDF) & 0.0 & 1.0 & 1.2 & 0.5 & 2.4 & 2.0 & 4.0 & 3.0 & 1.0 & 2.0 & 3.5 & 5.5 & 5.5 & 7.0 & 8.0 \\
\hline strategic petroleum reserve & 0.0 & 0.0 & 3.9 & 1.9 & 3.9 & 1.4 & 3.9 & 0.8 & 0.0 & 0.0 & 0.0 & 0.0 & 0.0 & 0.0 & 1.0 \\
\hline contingent $2 /$ & 0.0 & 0.0 & 0.0 & 0.0 & 0.0 & 0.0 & 0.0 & 0.0 & 0.0 & 0.0 & 3.8 & 0.0 & 7.6 & 0.0 & 0.0 \\
\hline Foreign & 46.5 & 33.8 & 27.3 & 19.0 & 41.0 & 28.4 & 54.7 & 37.9 & 15.6 & 31.2 & 46.7 & 62.3 & 62.3 & 71.6 & 82.3 \\
\hline Net lending & 0.6 & 0.6 & 1.2 & 0.9 & 1.7 & 3.5 & 2.3 & 4.0 & 1.3 & 16.3 & 17.8 & 1.0 & 18.7 & 1.0 & 1.0 \\
\hline Troop withdrawal & $\ldots$ & 10.9 & 0.0 & 0.0 & 0.0 & 0.0 & 0.0 & 0.0 & 0.0 & 0.0 & $\begin{array}{r}0.0 \\
0.0\end{array}$ & 0.0 & 0.0 & 0.0 & 1.0 \\
\hline Primary balance 3 / & -0.8 & -12.9 & -4.0 & -1.8 & 2.2 & -1.2 & 7.1 & -2.8 & -1.0 & -0.7 & -13.6 & 14.0 & -26.1 & -28.7 & -20.2 \\
\hline Domestic fiscal balance 4 / & -20.4 & -34.8 & -23.9 & -18.0 & -31.8 & -36.8 & -39.1 & -50.2 & -9.8 & -32.6 & -54.0 & -10.4 & -78.7 & -57.7 & -47.7 \\
\hline \multicolumn{16}{|l|}{ Overall deficit (payment order) } \\
\hline After grants & -9.9 & -15.6 & -10.8 & -7.3 & -6.8 & -26.4 & 8.0 & -22.3 & 1.8 & -9.9 & -16.3 & 6.4 & -22.3 & -8.1 & -11.5 \\
\hline Before grants & -71.9 & $\begin{array}{l}-130.0 \\
-74.8\end{array}$ & -54.4 & -40.6 & $\begin{array}{r}-0.0 \\
-77.7\end{array}$ & $\begin{array}{l}-20.4 \\
-70.6\end{array}$ & $\begin{array}{l}0.0 \\
-101.1\end{array}$ & -95.4 & $\begin{array}{r}1.0 \\
-27.3\end{array}$ & -68.2 & $\begin{array}{l}-106.6 \\
-106.6\end{array}$ & $\begin{array}{r}0.4 \\
-80.1\end{array}$ & -149.2 & $\begin{array}{r}-0.1 \\
-138.0\end{array}$ & $\begin{array}{l}-11.5 \\
-138.6\end{array}$ \\
\hline Change in arrears 5/ & -31.7 & -1.7 & -9.3 & -9.0 & -9.3 & 1.6 & -22.5 & -13.2 & -11.2 & -13.0 & -14.7 & -7.0 & -20.1 & -7.0 & -7.0 \\
\hline Domestic & -15.0 & -3.9 & -8.9 & -9.0 & -8.9 & 1.6 & -7.9 & -1.0 & -11.2 & -13.0 & -14.7 & -7.0 & -17.0 & -7.0 & -7.0 \\
\hline External & -16.7 & 2.2 & -0.4 & 0.0 & -0.4 & 0.0 & -14.6 & -12.1 & 0.0 & 0.0 & 0.0 & 0.0 & -3.1 & 0.0 & 0.0 \\
\hline Deficit (-) (cash basis) & -41.6 & -17.4 & -20.1 & -16.3 & -16.1 & -24.9 & -14.5 & -35.4 & -9.4 & -22.9 & -31.0 & -0.6 & -42.4 & -15.1 & -18.5 \\
\hline
\end{tabular}

CInternational Monetary Fund. Not for Redistribution 
Table 2. Rwanda: Operations of the Central Government, 2001-06 (concluded)

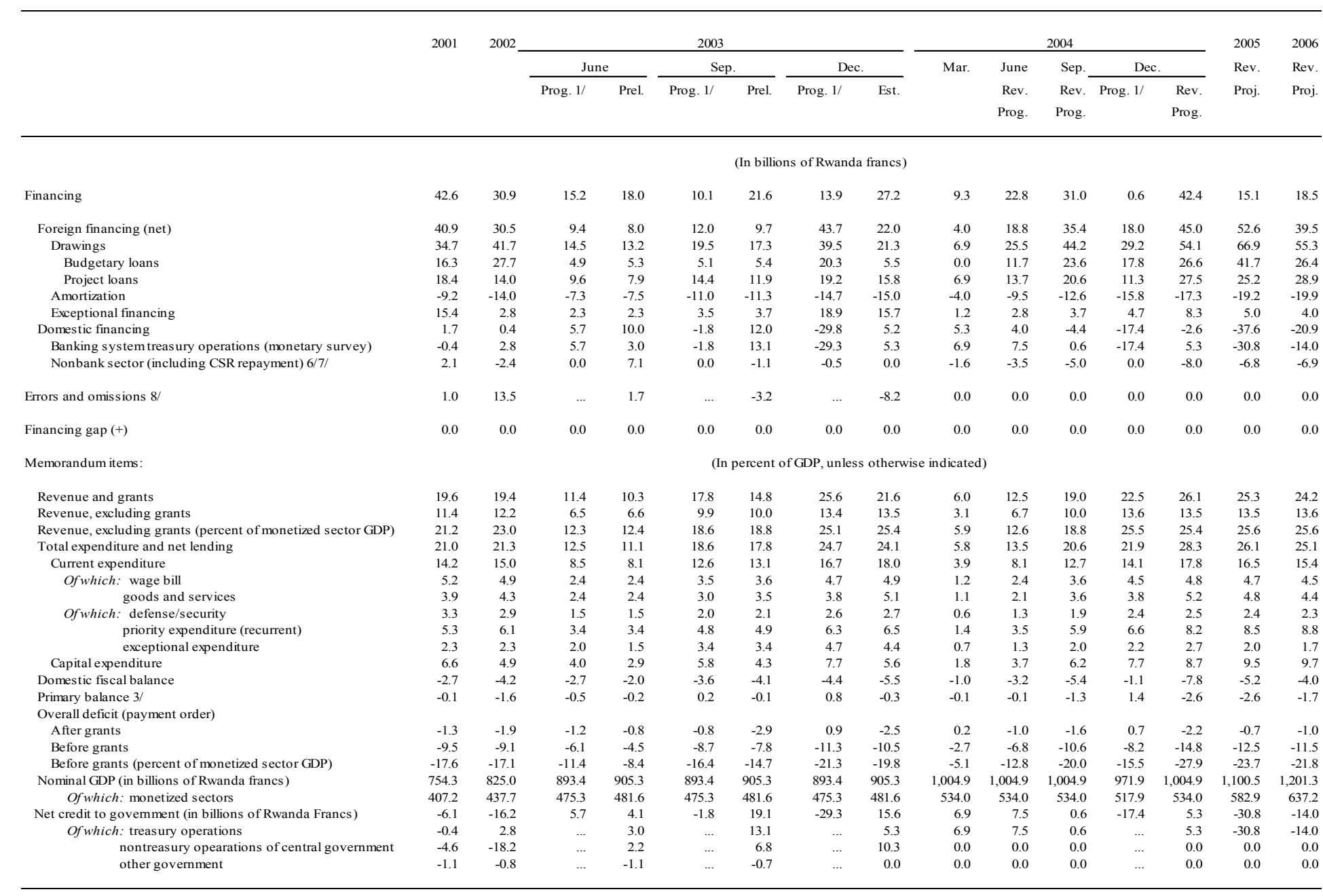

Sources: Rwandese authorities; and Fund staff estimates and projections.

1/ Program numbers refer to IMF Country Report No. 03/192.

2/ Planned to be executed only in case of sufficient additional external budgetary grants.

3/ Definition excludes exceptional expenditures; defined as total revenue (excluding privatization proceeds) minus noninterest current expenditure (excluding exceptional expenditure) minus domestically financed capital expenditure. In 2002, the defintion includes the cost of the troop withdrawa.

$5 /$ A

5/A A R - Caisse Sociale du Rwanda.

$7 /$ Includes RF 8.8 billion in termporac

8/ A negative number implies a diary credits from Union des Banques Populaires du Rwanda (UBPR) in June 2003.

8/ A negative number implies a discrepancy that is consistent with underestimation of financing.

\section{CInternational Monetary Fund. Not for Redistribution}


Table 3. Rwanda: Monetary Survey, 2002-04

$2001 \quad 20021$

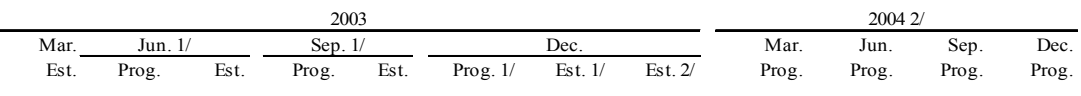

Monetary authorities

Net foreign assets

Foreign assets

Foreign liabilities

Net domestic asset

Domestic credit

Government (net)

Claims

Of which : overdraft

Deposits (excluding autonomous bodies)

Public nongovernment deposits (-)

Nongovernment credit

Private sector

Public enterprises

Commercial banks

Discount window

Money market ( $-=$ absorption)

Other items (net; asset + )

Reserve money 3/

Currency in circulation

Commercial bank reserves

Nonbank deposits

Of which: autonomous public agencies

Commercial banks

Net foreign assets

Foreign assets

Foreign liabilities

Reserves

NBR deposits

Required reserves

Excess reserves

Cash in vault

Net credit from NBR (rediscount; liability -)

Domestic credit

Government (net)

Credit

Deposits 3/

Public enterprises

Private sector

Other items (net; asset + )

Deposits

Private 4/

Public (nongovernment)

(In billions of Rwanda francs)

\begin{tabular}{|c|c|c|c|c|c|c|c|c|c|c|c|c|c|}
\hline 51.3 & 64.9 & 70.5 & 67.7 & 68.1 & 70.3 & 54.6 & 101.0 & 57.8 & 65.5 & 65.0 & 55.4 & 64.5 & 62.3 \\
\hline 97.1 & 111.6 & 123.1 & 120.3 & 123.2 & 122.8 & 105.8 & 153.1 & 109.7 & 124.3 & 123.7 & 114.2 & 123.3 & 121.0 \\
\hline 45.8 & 46.6 & 52.6 & 52.5 & 55.1 & 52.5 & 51.2 & 52.1 & 51.8 & 58.8 & 58.8 & 58.8 & 58.8 & 58.8 \\
\hline-11.1 & -20.0 & -24.0 & -22.4 & -21.6 & -24.9 & -4.5 & -54.1 & -7.4 & -15.1 & -12.0 & -1.4 & -8.6 & -6.0 \\
\hline 12.8 & -2.6 & -0.6 & 1.9 & 2.2 & -2.2 & 17.5 & -28.5 & 15.6 & 15.6 & 16.8 & 27.4 & 20.2 & 22.8 \\
\hline 16.0 & -5.2 & 1.9 & 4.8 & 3.5 & -0.3 & 15.3 & -27.1 & 12.7 & 12.7 & 19.6 & 20.2 & 13.3 & 18.0 \\
\hline 43.5 & 43.1 & 42.4 & 43.1 & 42.4 & 43.1 & 51.2 & 43.1 & 43.9 & 43.9 & 50.8 & 60.2 & 59.7 & 70.7 \\
\hline-- & -- & 0.0 & -- & - & -- & 8.8 & -- & 1.5 & 1.5 & -- & -- & -- & -- \\
\hline 27.5 & 48.2 & 40.5 & 38.2 & 38.9 & 43.3 & 35.9 & 70.2 & 31.2 & 31.2 & 31.2 & 40.0 & 46.4 & 52.7 \\
\hline-1.3 & -0.9 & -0.9 & -0.9 & -0.9 & -0.9 & -1.0 & -0.9 & -1.0 & -1.0 & -1.0 & -1.0 & -1.0 & -1.0 \\
\hline-1.9 & 3.5 & -1.6 & -2.0 & -0.3 & -1.0 & 3.1 & -0.4 & 3.9 & 3.9 & -1.8 & 8.2 & 7.8 & 5.8 \\
\hline 1.9 & 2.2 & 2.2 & 1.8 & 2.3 & 1.7 & 2.3 & 1.5 & 2.4 & 2.4 & 2.4 & 2.4 & 2.4 & 2.4 \\
\hline 0.1 & 0.1 & 0.1 & 0.1 & 0.1 & 0.1 & 0.1 & 0.1 & 0.1 & 0.1 & 0.1 & 0.1 & 0.1 & 0.1 \\
\hline-3.9 & 1.2 & -3.9 & -3.9 & -2.7 & -2.8 & 0.7 & -2.0 & 1.4 & 1.4 & -4.3 & 5.7 & 5.3 & 3.3 \\
\hline 1.5 & 1.2 & 1.2 & 1.2 & 1.3 & 1.2 & 1.4 & 1.2 & 1.4 & 1.4 & 1.0 & 1.0 & 1.0 & 1.4 \\
\hline-5.4 & -0.1 & -5.1 & -5.1 & -4.0 & -4.0 & -0.7 & -3.2 & -- & -- & -5.3 & 4.7 & 4.3 & 1.9 \\
\hline-24.0 & -17.4 & -23.4 & -24.3 & -23.9 & -22.7 & -22.0 & -25.6 & -23.0 & -30.7 & -28.8 & -28.8 & -28.8 & -28.8 \\
\hline 40.2 & 44.9 & 46.5 & 45.3 & 46.5 & 44.1 & 50.1 & 46.9 & 50.4 & 50.4 & 53.0 & 54.0 & 55.9 & 56.3 \\
\hline 25.8 & 28.0 & 26.4 & 27.1 & 29.4 & 26.9 & 32.1 & 29.0 & 34.1 & 34.1 & 34.6 & 35.1 & 36.9 & 37.6 \\
\hline 10.7 & 13.2 & 15.5 & 14.5 & 12.9 & 14.7 & 14.9 & 14.2 & 13.4 & 13.4 & 15.4 & 15.9 & 16.0 & 15.8 \\
\hline 3.7 & 3.7 & 4.6 & 3.7 & 4.3 & 3.7 & 3.2 & 3.7 & 3.0 & 3.0 & 3.0 & 3.0 & 3.0 & 3.0 \\
\hline 1.1 & 1.0 & 1.3 & 1.0 & 0.7 & 1.0 & 0.4 & 1.0 & 0.7 & 0.7 & -- & -- & -- & 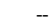 \\
\hline
\end{tabular}

\begin{tabular}{|c|c|c|c|c|c|c|c|c|c|c|c|c|c|}
\hline 29.7 & 27.9 & 32.9 & 33.0 & 32.1 & 35.5 & 29.4 & 33.5 & 34.0 & 38.7 & 37.5 & 44.5 & 39.7 & 41.9 \\
\hline 34.3 & 35.0 & 39.8 & 41.0 & 43.3 & 43.4 & 39.2 & 41.5 & 43.9 & 49.8 & 48.7 & 55.6 & 50.8 & 53.0 \\
\hline 4.6 & 7.1 & 6.8 & 8.0 & 11.3 & 8.0 & 9.8 & 8.0 & 10.0 & 11.1 & 11.1 & 11.1 & 11.1 & 11.1 \\
\hline 10.7 & 13.2 & 15.5 & 14.5 & 12.9 & 14.7 & 14.9 & 14.2 & 13.4 & 13.4 & 15.4 & 16.0 & 16.1 & 15.8 \\
\hline 7.9 & 10.5 & 11.0 & 11.5 & 8.3 & 11.5 & 11.6 & 11.3 & 11.0 & 11.0 & 13.2 & 13.6 & 13.7 & 13.4 \\
\hline 8.4 & 9.7 & 10.2 & 9.2 & 10.8 & 9.2 & 11.4 & 9.4 & 11.9 & 11.9 & 11.6 & 12.0 & 12.0 & 12.4 \\
\hline-0.5 & 0.8 & 0.8 & 2.3 & -2.4 & 2.3 & 0.1 & 1.9 & -0.9 & -0.9 & 1.6 & 1.6 & 1.7 & 1.0 \\
\hline 2.8 & 2.7 & 4.4 & 3.0 & 4.5 & 3.2 & 3.3 & 2.8 & 2.4 & 2.4 & 2.4 & 2.4 & 2.4 & 2.4 \\
\hline 3.9 & -1.2 & 3.9 & 3.7 & 2.7 & 2.5 & -0.7 & 2.0 & -1.4 & -1.4 & 4.3 & -5.7 & -5.3 & -3.3 \\
\hline 78.4 & 93.7 & 85.2 & 90.0 & 91.6 & 88.1 & 103.8 & 94.0 & 104.4 & 104.4 & 101.5 & 108.9 & 113.3 & 115.2 \\
\hline 2.1 & 7.1 & 3.6 & 2.9 & 2.5 & 0.3 & 5.7 & -0.2 & 4.8 & 4.8 & 4.8 & 4.8 & 4.8 & 4.8 \\
\hline 8.2 & 14.7 & 13.9 & 10.9 & 20.5 & 9.4 & 16.6 & 8.0 & 15.2 & 15.2 & 15.2 & 15.2 & 15.2 & 15.2 \\
\hline 6.1 & 7.6 & 10.3 & 8.0 & 18.0 & 9.1 & 10.9 & 8.2 & 10.4 & 10.4 & 10.4 & 10.4 & 10.4 & 10.4 \\
\hline 0.8 & 3.4 & 2.3 & 2.3 & 3.2 & 2.3 & 4.6 & 2.3 & 4.8 & 4.8 & 4.8 & 4.8 & 4.8 & 4.8 \\
\hline 75.5 & 83.3 & 79.3 & 84.8 & 85.8 & 85.5 & 93.4 & 91.9 & 94.8 & 94.8 & 91.9 & 99.4 & 103.7 & 105.7 \\
\hline-21.9 & -23.4 & -25.2 & -22.8 & -27.9 & -22.6 & -27.1 & -22.2 & -24.7 & -25.0 & -24.7 & -24.7 & -24.7 & -24.7 \\
\hline 100.7 & 110.2 & 112.4 & 118.4 & 111.4 & 118.2 & 120.3 & 121.4 & 125.6 & 130.0 & 134.0 & 139.0 & 139.0 & 144.9 \\
\hline 85.5 & 94.8 & 96.4 & 102.9 & 96.3 & 103.0 & 99.3 & 105.8 & 105.8 & 110.1 & 113.1 & 116.9 & 115.8 & 125.1 \\
\hline 15.2 & 15.4 & 16.0 & 15.4 & 15.1 & 15.4 & 21.0 & 15.4 & 19.9 & 19.9 & 21.0 & 22.1 & 23.2 & 19.9 \\
\hline
\end{tabular}

CInternational Monetary Fund. Not for Redistribution 
Table 3. Rwanda: Monetary Survey, 2002-04 (concluded)

\begin{tabular}{|c|c|c|c|c|c|c|c|c|c|c|c|c|c|c|}
\hline & \multirow[t]{3}{*}{2001} & \multirow[t]{3}{*}{$20021 /$} & \multicolumn{8}{|c|}{2003} & \multicolumn{4}{|c|}{$20042 /$} \\
\hline & & & \multirow{2}{*}{$\begin{array}{c}\text { Mar. } \\
\text { Est. }\end{array}$} & \multicolumn{2}{|c|}{ Jun. 1/ } & \multicolumn{2}{|c|}{ Sep. 1/ } & \multicolumn{3}{|c|}{ Dec. } & \multirow{2}{*}{$\begin{array}{l}\text { Mar. } \\
\text { Prog. }\end{array}$} & \multirow{2}{*}{$\begin{array}{l}\text { Jun. } \\
\text { Prog. }\end{array}$} & \multirow{2}{*}{$\begin{array}{l}\text { Sep. } \\
\text { Prog. }\end{array}$} & \multirow{2}{*}{$\begin{array}{r}\text { Dec. } \\
\text { Prog. }\end{array}$} \\
\hline & & & & Prog. & Est. & Prog. & $\overline{\text { Est. }}$ & Prog. 1/ & Est. 1/ & $\overline{\text { Est. } 2 /}$ & & & & \\
\hline Monetary survey & \multicolumn{14}{|c|}{ (In billions of Rwanda francs) } \\
\hline Net foreign assets & 81.0 & 92.8 & 103.5 & 100.8 & 100.2 & 105.7 & 84.0 & 134.5 & 91.8 & 104.2 & 102.5 & 99.9 & 104.3 & 104.2 \\
\hline Net domestic assets & 49.3 & 49.2 & 40.0 & 49.9 & 44.6 & 43.4 & 71.5 & 20.9 & 70.9 & 62.9 & 69.1 & 77.2 & 74.6 & 81.3 \\
\hline Domestic credit & 95.1 & 90.0 & 88.6 & 95.7 & 96.6 & 88.7 & 120.5 & 67.5 & 118.6 & 118.6 & 122.6 & 130.7 & 128.1 & 134.7 \\
\hline Government (net) & 18.1 & 1.9 & 5.5 & 7.7 & 6.0 & 0.1 & 21.0 & -27.4 & 17.5 & 17.5 & 24.4 & 25.0 & 18.1 & 22.8 \\
\hline Public nongovernment deposits (-) & -1.3 & -0.9 & -0.9 & -0.9 & -0.9 & -0.9 & -1.0 & -0.9 & -1.0 & -1.0 & -1.0 & -1.0 & -1.0 & -1.0 \\
\hline Public enterprises & 1.0 & 3.5 & 2.4 & 2.4 & 3.3 & 2.4 & 4.8 & 2.4 & 4.9 & 4.9 & 4.9 & 4.9 & 4.9 & 4.9 \\
\hline Private sector $3 /$ & 77.4 & 85.5 & 81.5 & 86.6 & 88.1 & 87.1 & 95.7 & 93.4 & 97.2 & 97.2 & 94.3 & 101.8 & 106.1 & 108.1 \\
\hline Other items (net; asset + ) & -45.9 & -40.8 & -48.6 & -45.9 & -52.0 & -45.3 & -49.1 & -46.6 & -47.7 & -55.7 & -53.5 & -53.5 & -53.5 & -53.5 \\
\hline Broad money & 130.2 & 142.0 & 143.4 & 150.4 & 144.7 & 148.9 & 155.5 & 155.4 & 162.7 & 167.1 & 171.6 & 177.1 & 178.9 & 185.4 \\
\hline Currency in circulation & 25.8 & 28.0 & 26.4 & 28.3 & 29.4 & 26.9 & 32.1 & 30.3 & 34.1 & 34.1 & 34.6 & 35.1 & 36.9 & 37.6 \\
\hline Deposits 3/ & 104.4 & 113.9 & 117.0 & 122.8 & 115.4 & 122.0 & 123.5 & 125.1 & 128.6 & 133.0 & 137.0 & 142.0 & 142.0 & 147.9 \\
\hline \multirow{2}{*}{ Of which: foreign currency deposits } & 27.9 & 26.3 & 30.1 & 32.1 & 34.7 & 34.0 & 35.2 & 31.9 & 37.1 & 41.5 & 38.3 & 39.5 & 40.7 & 41.9 \\
\hline & \multicolumn{14}{|c|}{ (Annual changes in percent of beginning-of-period broad money) } \\
\hline Net foreign assets & 11.8 & 9.1 & 20.0 & 23.9 & 15.9 & 26.8 & 4.7 & 25.0 & -8.2 & 0.3 & -10.0 & -9.1 & 5.6 & 0.0 \\
\hline Net domestic assets & -2.6 & -0.1 & -11.0 & -11.1 & -10.1 & -14.1 & 10.6 & -17.0 & 20.4 & 14.8 & 26.4 & 27.5 & 6.0 & 11.0 \\
\hline Domestic credit & 0.3 & -4.0 & -7.9 & -4.8 & -4.1 & -10.3 & 13.5 & -15.5 & 19.7 & 19.7 & 23.1 & 22.8 & 4.7 & 9.7 \\
\hline Government (net) & -5.1 & -12.4 & -12.8 & -10.2 & -11.2 & -15.0 & 0.7 & -20.2 & 10.7 & 10.7 & 12.8 & 12.7 & -1.8 & 3.2 \\
\hline Economy & 5.4 & 8.5 & 4.8 & 5.4 & 7.1 & 4.8 & 12.8 & 4.7 & 9.0 & 9.0 & 10.3 & 10.1 & 6.6 & 6.5 \\
\hline Other items (net; asset + ) & -2.9 & 3.9 & -3.1 & -6.3 & -6.1 & -3.9 & -2.9 & -1.5 & 0.7 & -4.9 & 3.3 & 4.7 & 1.3 & 1.3 \\
\hline Broad money & 9.2 & 9.0 & 9.0 & 12.8 & 5.8 & 12.7 & 15.3 & 8.0 & 12.2 & 15.2 & 16.4 & 18.3 & 11.6 & 11.0 \\
\hline & \multicolumn{14}{|c|}{ (Annual percent changes) } \\
\hline Net foreign assets & 21.1 & 14.6 & 34.1 & 45.0 & 27.8 & 48.7 & 8.1 & 34.9 & -1.1 & 0.5 & -12.6 & -12.0 & 9.4 & 0.0 \\
\hline Net domestic assets & -5.9 & -0.2 & -32.6 & -23.6 & -29.8 & -31.5 & 15.4 & -59.6 & 44.2 & 52.1 & 72.9 & 73.2 & 4.4 & 29.3 \\
\hline Domestic credit & 0.4 & -5.4 & -10.6 & -6.3 & -5.4 & -13.4 & 17.8 & -24.9 & 31.8 & 31.8 & 38.4 & 35.3 & 6.3 & 13.6 \\
\hline Government (net) & -25.1 & -89.4 & -75.2 & -64.0 & -71.7 & -99.6 & 4.7 & $-1,525.8$ & 810.6 & 810.6 & 339.4 & 313.9 & -14.1 & 30.3 \\
\hline Economy & 9.1 & 14.3 & 8.3 & 8.9 & 12.0 & 7.7 & 21.0 & 7.8 & 14.8 & 14.8 & 18.3 & 16.7 & 10.6 & 10.7 \\
\hline Other items (net; asset + ) & 8.2 & -11.1 & 9.1 & 21.4 & 19.0 & 12.1 & 8.6 & 4.5 & 16.9 & 14.6 & -8.4 & -12.0 & -3.8 & -4.0 \\
\hline & \multicolumn{14}{|c|}{ (In percent, unless otherwise indicated) } \\
\hline \multicolumn{15}{|l|}{ Memorandum items: } \\
\hline Currency/broad money ratio & 19.8 & 19.8 & 18.4 & 18.7 & 20.3 & 17.9 & 20.6 & 19.3 & 21.0 & 20.4 & 20.2 & 19.8 & 20.6 & 20.3 \\
\hline Reserve money annual growth & 6.6 & 11.9 & 5.8 & 12.6 & 12.5 & 13.9 & 26.0 & 7.1 & 12.2 & 12.2 & 14.0 & 16.2 & 11.5 & 11.6 \\
\hline Reserves/deposits & 39.9 & 40.8 & 41.4 & 39.1 & 41.7 & 38.0 & 41.7 & 39.2 & 40.1 & 38.8 & 39.5 & 38.8 & 40.2 & 38.8 \\
\hline Money multiplier & 3.2 & 3.2 & 3.1 & 3.2 & 3.1 & 3.3 & 3.1 & 3.3 & 3.2 & 3.3 & 3.2 & 3.3 & 3.2 & 3.3 \\
\hline Velocity of broad money (end of period) & $\ldots$ & $\ldots$ & $\ldots$ & $\ldots$ & $\ldots$ & $\ldots$ & $\ldots$ & 5.7 & 5.6 & 5.4 & $\ldots$ & $\ldots$ & $\ldots$ & 5.4 \\
\hline Velocity of broad money (average of period) & $\ldots$ & $\ldots$ & $\ldots$ & $\ldots$ & $\ldots$ & $\ldots$ & $\ldots$ & 5.9 & 7.0 & 7.0 & $\ldots$ & $\ldots$ & $\ldots$ & 6.3 \\
\hline Net open position of the NBR (in RF billion) & 51.2 & 62.3 & 68.3 & 66.6 & 65.0 & 69.8 & 51.3 & 102.4 & 54.1 & 61.4 & 61.9 & 52.2 & 61.3 & 58.9 \\
\hline Net open position of commercial banks (in RF billion) & 1.9 & 4.3 & 5.1 & 3.6 & 0.5 & 4.2 & -2.4 & 4.3 & 0.6 & 1.3 & -0.8 & 5.0 & -1.0 & 0.0 \\
\hline Extended broad money 4/ & $\ldots$ & $\ldots$ & $\ldots$ & $\ldots$ & 166.8 & $\ldots$ & 179.0 & $\ldots$ & 183.7 & 188.1 & 193.8 & 200.0 & 202.0 & 208.8 \\
\hline Nominal GDP (in RF billion) & $\ldots$ & $\ldots$ & $\ldots$ & $\ldots$ & $\ldots$ & $\ldots$ & $\ldots$ & 893.4 & 905.3 & 905.3 & $\ldots$ & $\ldots$ & $\ldots$ & $1,004.9$ \\
\hline
\end{tabular}

Source: National Bank of Rwanda (NBR)

$1 /$ Converted at the program exchange rate for 2003 (equal to the end-year rate 2002) of Rwanda franc (RF) 511.9/U.S. dollar.

$2 /$ Converted at the program exchange rate for 2004 (equal to the end-year rate 2003) of RF 580.3/U.S. dollar.

3 / The definition of reserve money as performance criteria or structural benchmark differs from the definition in the monetary program

in that it excludes the deposits of a defunct savings bank, import deposits, and dormant accounts.

For 2002 and 2003, the PC/benchmark is based on a three-week average, while data in this table refer to the last day of the period.

4/ Broad money plus deposits in UBPR and Rwanda Development Bank (BRD). 
Table 4. Rwanda: Balance of Payments, 2001-06 (In millions of U.S. dollars, unless otherwise indicated)

\begin{tabular}{|c|c|c|c|c|c|c|c|c|c|}
\hline & \multirow[t]{2}{*}{2001} & \multicolumn{2}{|c|}{2002} & \multicolumn{3}{|c|}{2003} & \multirow{2}{*}{$\begin{array}{c}2004 \\
\text { Rev. } \\
\text { Prog. }\end{array}$} & \multirow{2}{*}{$\begin{array}{l}2005 \\
\text { Proj. }\end{array}$} & \multirow{2}{*}{$\begin{array}{l}2006 \\
\text { Proj. }\end{array}$} \\
\hline & & Prog. & Est. & PRGF & Prog. & $\overline{\text { Est. }}$ & & & \\
\hline Exports, foo.b. & 93.5 & 77.5 & 67.3 & 87.0 & 71.8 & 63.0 & 68.0 & 79.6 & 88.9 \\
\hline Of which: coffee & 19.4 & 22.6 & 14.6 & 28.9 & 16.8 & 15.0 & 16.7 & 25.2 & 31.5 \\
\hline tea & 22.7 & 23.6 & 22.0 & 24.8 & 24.6 & 22.5 & 24.6 & 27.0 & 30.4 \\
\hline Imports, f.o.b. & 237.9 & 256.5 & 234.7 & 264.7 & 253.6 & 244.0 & 283.0 & 303.1 & 321.7 \\
\hline Trade balance & -144.4 & -179.0 & -167.4 & -177.7 & -181.8 & -181.0 & -214.9 & -223.4 & -232.9 \\
\hline Services (net) & -125.3 & -120.8 & -125.1 & -120.1 & -140.0 & -157.0 & -161.3 & -164.5 & -172.7 \\
\hline Income & -22.5 & -24.6 & -19.1 & -25.4 & -24.3 & -25.1 & -21.9 & -22.2 & -15.8 \\
\hline Of which: interest on public debt $1 /$ & -12.6 & -12.2 & -13.5 & -11.6 & -14.6 & -14.0 & -14.3 & -14.7 & -14.3 \\
\hline Current transfers (net) $2 /$ & 191.9 & 124.0 & 194.8 & 106.8 & 152.7 & 221.9 & 280.5 & 262.1 & 240.6 \\
\hline Private & 21.7 & 23.0 & 24.5 & 24.4 & 26.0 & 29.4 & 30.9 & 32.5 & 34.3 \\
\hline Public 2/3/ & 170.1 & 101.0 & 170.3 & 82.5 & 126.8 & 192.5 & 249.6 & 229.6 & 206.3 \\
\hline Of which: HIPC Initiative grants & 23.9 & 10.5 & 25.6 & 0.0 & 4.7 & 23.0 & 27.2 & 32.0 & 38.8 \\
\hline Current account balance (including official trans fers) & -100.3 & -200.4 & -116.7 & -216.4 & -193.4 & -141.2 & -117.7 & -148.2 & -180.7 \\
\hline Current account balance (excluding official transfers) & -270.5 & -301.4 & -287.0 & -298.8 & -320.2 & -333.7 & -367.3 & -377.7 & -387.0 \\
\hline Capital account & 67.0 & 68.8 & 41.6 & 74.3 & 67.8 & 41.1 & 59.4 & 76.7 & 85.5 \\
\hline Capital trans fers & 63.4 & 68.8 & 41.6 & 74.3 & 67.8 & 41.1 & 59.4 & 76.7 & 85.5 \\
\hline Debt forgiveness & 3.6 & 0.0 & 0.0 & 0.0 & 0.0 & 0.0 & 0.0 & 0.0 & 0.0 \\
\hline Principal not yet due forgiven & 3.6 & 0.0 & 0.0 & 0.0 & 0.0 & 0.0 & 0.0 & 0.0 & 0.0 \\
\hline Financial account & 78.3 & 11.4 & 66.6 & 33.5 & 29.7 & 23.2 & 46.0 & 112.8 & 126.4 \\
\hline Direct investment & 3.8 & 4.8 & 2.6 & 5.0 & 2.7 & 2.1 & 14.0 & 16.1 & 18.5 \\
\hline Public sector capital & 55.4 & 11.1 & 54.5 & 10.0 & 14.7 & 11.9 & 62.4 & 78.7 & 56.8 \\
\hline Long-term borrowing 3/4/ & 78.4 & 37.3 & 83.9 & 40.2 & 42.8 & 39.9 & 91.9 & 110.4 & 88.6 \\
\hline Principal not yet due rescheduled 5/ & 8.8 & 0.0 & 0.0 & 0.0 & 6.7 & 6.7 & 0.0 & 0.0 & 0.0 \\
\hline Scheduled amortization 6/ & 19.4 & 26.2 & 29.4 & 30.3 & 28.0 & 28.0 & 29.5 & 31.7 & 31.8 \\
\hline Principal not yet due 5 / & 12.4 & 0.0 & 0.0 & 0.0 & 6.7 & 6.7 & 0.0 & 0.0 & 0.0 \\
\hline Other capital 7/ & 19.1 & -4.5 & 9.5 & 18.5 & 12.2 & 9.1 & -30.4 & 18.0 & 51.1 \\
\hline Capital and financial account balance & 145.3 & 80.2 & 108.1 & 107.8 & 97.5 & 64.3 & 105.4 & 189.5 & 211.9 \\
\hline Errors and omissions & -20.6 & 0.0 & 27.9 & 0.0 & 0.0 & 40.0 & 0.0 & 0.0 & 0.0 \\
\hline Overall balance & 24.4 & -120.1 & 19.3 & -108.6 & -96.0 & -36.9 & -12.3 & 41.3 & 31.2 \\
\hline Financing & -24.4 & -27.3 & -19.2 & -11.6 & -75.2 & 36.9 & 0.9 & -49.5 & -37.6 \\
\hline Change in net foreign assets of NBR (increase -) & -25.1 & -5.0 & -29.7 & -11.6 & -55.6 & 28.8 & 3.6 & -49.5 & -37.6 \\
\hline Net credit from the Fund & 1.5 & 5.8 & -6.0 & 0.1 & -0.1 & -0.9 & -3.6 & -8.1 & -14.7 \\
\hline Disbursements/purchases & 12.1 & -0.7 & 0.7 & -1.4 & 1.6 & 0.8 & 1.7 & 1.7 & 0.8 \\
\hline Repayments/repurchases & -10.6 & 6.5 & -6.7 & 1.5 & -1.6 & -1.7 & -5.3 & -9.8 & -15.5 \\
\hline Change in other gross official reserves (increase -) & -21.4 & -10.8 & -31.6 & -11.7 & -55.5 & 29.4 & 5.7 & -43.0 & -24.6 \\
\hline Change in other foreign liabilities (increase + ) & -5.3 & 0.0 & 7.9 & 0.0 & 0.0 & 0.3 & 1.5 & 1.6 & 1.7 \\
\hline Change in arrears (decrease -) 8 / & -37.8 & -22.3 & 4.6 & 0.0 & -27.9 & -22.6 & -5.3 & 0.0 & 0.0 \\
\hline Accumulation & 2.9 & 0.0 & 5.6 & 0.0 & 0.0 & 1.3 & 0.0 & 0.0 & 0.0 \\
\hline Reduction & 40.8 & -22.3 & 1.0 & 0.0 & 27.9 & 23.8 & 5.3 & 0.0 & 0.0 \\
\hline Exceptional financing 9/ & 38.6 & 0.0 & 5.9 & 0.0 & 8.3 & 30.7 & 2.7 & 0.0 & 0.0 \\
\hline Cancellation & 13.4 & 0.0 & 0.6 & 0.0 & 0.6 & 0.6 & 0.3 & 0.0 & 0.0 \\
\hline Stock of arrears & 12.8 & 0.0 & 0.0 & 0.0 & 0.0 & 0.0 & 0.0 & 0.0 & 0.0 \\
\hline Current debt service & 0.6 & 0.0 & 0.6 & 0.0 & 0.6 & 0.6 & 0.3 & 0.0 & 0.0 \\
\hline Rescheduling & 25.2 & 0.0 & 5.3 & 0.0 & 7.7 & 30.1 & 2.4 & 0.0 & 0.0 \\
\hline Stock of arrears & 21.8 & 0.0 & 0.0 & 0.0 & 2.9 & 23.1 & 0.0 & 0.0 & 0.0 \\
\hline Current debt service & 3.4 & 0.0 & 5.3 & 0.0 & 4.8 & 7.0 & 2.4 & 0.0 & 0.0 \\
\hline Financing need & 0.0 & 147.4 & 0.0 & 120.2 & 171.2 & 0.0 & -11.4 & -8.2 & -6.4 \\
\hline Identified financing $10 /$ & 0.0 & 147.4 & 0.0 & 120.2 & 171.2 & 0.0 & 11.4 & 8.2 & 6.4 \\
\hline Of which: exceptional financing (assumed) 11/ & 0.0 & 29.8 & 0.0 & 9.8 & 27.7 & 0.0 & 11.4 & 8.2 & 6.4 \\
\hline Financing gap $12 /$ & 0.0 & 0.0 & 0.0 & 0.0 & 0.0 & 0.0 & 0.0 & 0.0 & 0.0 \\
\hline \multicolumn{10}{|l|}{ Memorandum items: } \\
\hline \multicolumn{10}{|l|}{ Current account deficit (in percent of GDP) } \\
\hline Excluding official transfers & -15.9 & -17.0 & -16.6 & -15.7 & -18.7 & -19.8 & -21.4 & -20.8 & -20.1 \\
\hline Including official transfers $3 /$ & -5.9 & -11.3 & -6.7 & -11.4 & -11.3 & -8.4 & -6.9 & -8.2 & -9.4 \\
\hline External budgetary assistance to central government (in percent of GDP) & 6.7 & 7.2 & 7.9 & 5.8 & 10.5 & 6.3 & 11.7 & 11.4 & 8.3 \\
\hline Gross official reserves & 212.1 & 223.6 & 243.6 & 236.6 & 299.1 & 214.2 & 208.6 & 251.5 & 276.2 \\
\hline Gross official reserves (in months of imports of goods and services) & 6.0 & 5.9 & 6.3 & 6.2 & 7.4 & 5.0 & 4.6 & 5.2 & 5.5 \\
\hline Overall balance (in percent of GDP) & 1.4 & -6.8 & 1.1 & -5.7 & -5.6 & -2.2 & -0.7 & 2.3 & 1.6 \\
\hline Total external debt $13 /$ & $1,336.1$ & $1,295.1$ & $1,401.0$ & $1,268.4$ & $1,471.4$ & $1,432.8$ & $1,485.5$ & $1,556.2$ & $1,510.0$ \\
\hline Total external debt (in percent of GDP) & 78.4 & 73.2 & 80.9 & 66.8 & 86.1 & 85.1 & 86.6 & 85.7 & 78.5 \\
\hline Scheduled debt service ratio after HIPC Initiative debt relief 14/ & 9.3 & 15.4 & 13.8 & 9.1 & 9.1 & 10.3 & 9.6 & 12.7 & 10.5 \\
\hline
\end{tabular}

Sources: Rwandese authorities; and staff estimates and projections.

1/ Including interest due to the Fund.

2/ Current transfers include budgetary and HIPC Initiative grants, and humanitarian and technical assistance.

3/ In the "Prog." and "PRGF" columns for 2002 and 2003, official transfers and/or budgetary loans exclude expected but not yet disbursed budgetary assistance.

4/ Includes disbursed project and budgetary loans through end-2003 and committed loans thereafter. For 2004, including two tranches of a World Bank IRC credit (US\$ 40 million),

for which the conditionality has been substantially met.

5/ In 2003, representing the restructuring of loans by the OPEC Fund. The restructuring of one such loan is part of the HIPC relief granted by the OPEC Fund, resulting in an NPV reduction of $\$$ 6/ Excluding Fund.

7/ Other capital includes long-term private capital, commercial credit, the change in the net foreign assets of commercial banks, and unrecorded imports.

8/ The program assumes clearance of all outstanding arrears (including arrears to non-Paris Club creditors) in 2004

9/ Signed rescheduling and cancellation, as well as reschedulings expected to be signed with Paris Club creditors before the end of the June 30, 2004 deadline for the conclusion of bilateral agre 10/ Data in the "Prog." and "PRGF" columns for 2002 and 2003 include expected but not yet disbursed budgetary assistance.

11/ Tentative rescheduling by non-Paris Club creditors. For the years 2004-06, it also includes assumed debt relief by Paris Club creditors on Cologne terms, in the form of flow rescheduling delivered after the end of the current consolidation period. Following the completion point, the data for "debt service due" will be revised to reflect the impact of the stock-of-debt operation.

12/ The financing gap refers to the difference between the overall balance and the identified financing (actual and expected).

13/ After rescheduling, including arrears and new debt (the latter includes assumed project and budgetary disbursements for the period 2004-06).

The data under the "PRGF" and the "Prog."/"Est." columns are not comparable since they are based on a different treatment of projected loan disbursements.

Data are preliminary and do not reflect changes in exchange rates.

$14 /$ In percent of exports of goods and services. Data are preliminary. 
Table 5. Rwanda: External Financing Requirement and Sources, 2001-06 (In millions of U.S. dollars, unless otherwise indicated)

\begin{tabular}{|c|c|c|c|c|c|c|}
\hline & 2001 & 2002 & 2003 & 2004 & 2005 & 2006 \\
\hline Resource balance & -269.7 & -292.5 & -338.0 & -376.2 & -388.0 & -405.6 \\
\hline Exports goods and nonfactor services & 157.1 & 132.7 & 126.2 & 137.7 & 159.7 & 174.9 \\
\hline Imports goods and nonfactor services & 426.8 & 425.2 & 464.2 & 513.9 & 547.6 & 580.5 \\
\hline $\begin{array}{l}\text { Noninterest current account } \\
\quad \text { (excluding official transfers) } 1 /\end{array}$ & -257.9 & -273.5 & -319.7 & -352.9 & -363.0 & -372.7 \\
\hline Scheduled interest & -12.6 & -13.5 & -14.0 & -14.3 & -14.7 & -14.3 \\
\hline Capital and financial account $2 /$ & -26.0 & 10.6 & 16.6 & -45.9 & 2.4 & 37.8 \\
\hline Scheduled amortization & -19.4 & -29.4 & -28.0 & -29.5 & -31.7 & -31.8 \\
\hline Other capital & -6.6 & 40.0 & 44.6 & -16.4 & 34.1 & 69.6 \\
\hline Increase in net official reserves (excluding IMF -) & -26.7 & -23.7 & 29.7 & 7.2 & -41.4 & -23.0 \\
\hline IMF repurchases/repayments & -10.6 & -6.7 & -1.7 & -5.3 & -9.8 & -15.5 \\
\hline Changes in arrears (decrease $=-$ ) & -37.8 & 4.6 & -22.6 & -5.3 & $\ldots$ & $\ldots$ \\
\hline External financing requirements & -371.5 & -302.3 & -311.6 & -416.6 & -426.5 & -387.7 \\
\hline Disbursements-Existing & 324.1 & 296.5 & 274.3 & 235.0 & 210.1 & 220.0 \\
\hline Grants (project and nonproject) & 233.6 & 211.9 & 233.6 & 188.1 & 168.5 & 173.7 \\
\hline Project (Public Investment Program) & 63.4 & 41.6 & 41.1 & 59.4 & 76.7 & 85.5 \\
\hline Nonproject 3/ & 170.1 & 170.3 & 192.5 & 128.7 & 91.8 & 88.2 \\
\hline Central government & 76.1 & 81.0 & 93.9 & 33.1 & & \\
\hline Other & 94.0 & 89.3 & 98.6 & 95.5 & 91.8 & 88.2 \\
\hline Loans (project and nonproject) & 78.4 & 83.9 & 39.9 & 46.9 & 41.6 & 46.3 \\
\hline IDA & 52.5 & 77.1 & 25.4 & 42.9 & 36.4 & 40.0 \\
\hline Project & 21.7 & 26.5 & 20.7 & 42.9 & 36.4 & 40.0 \\
\hline Nonproject 4/ & 30.8 & 50.6 & 4.7 & & & \\
\hline Other multilateral banks & 25.9 & 6.8 & 14.4 & 4.0 & 5.2 & 6.3 \\
\hline Project & 19.9 & 2.8 & 8.7 & 4.0 & 5.2 & 6.3 \\
\hline Nonproject & 6.0 & 4.0 & 5.7 & $\ldots$ & $\ldots$ & $\ldots$ \\
\hline Purchases from IMF & 12.1 & 0.7 & 0.8 & $\ldots$ & $\ldots$ & $\ldots$ \\
\hline Exceptional financing (signed) & 38.6 & 5.9 & 30.7 & 2.7 & $\ldots$ & $\ldots$ \\
\hline Principal not yet due rescheduled 5/ & 8.8 & $\ldots$ & 6.7 & $\ldots$ & $\ldots$ & $\ldots$ \\
\hline Disbursements-Expected & $\ldots$ & $\ldots$ & $\ldots$ & 167.6 & 208.3 & 161.2 \\
\hline Grants & $\ldots$ & $\ldots$ & $\ldots$ & 120.9 & 137.8 & 118.1 \\
\hline Project (public investment program) 6/ & $\ldots$ & $\ldots$ & $\ldots$ & & & \\
\hline Budgetary assistance (central government) & $\cdots$ & $\begin{array}{l}\cdots \\
\cdots\end{array}$ & $\begin{array}{l}\cdots \\
\cdots\end{array}$ & 120.9 & 137.8 & 118.1 \\
\hline Loans & $\ldots$ & $\ldots$ & $\ldots$ & 45.0 & 68.8 & 42.3 \\
\hline IDA & $\ldots$ & $\ldots$ & $\ldots$ & 40.0 & 50.0 & 30.0 \\
\hline Project (public investment program) 6/ & $\ldots$ & $\ldots$ & $\ldots$ & & & \\
\hline Nonproject & $\ldots$ & $\ldots$ & $\ldots$ & 40.0 & 50.0 & 30.0 \\
\hline Other multilateral banks (excluding IMF) & $\ldots$ & $\ldots$ & $\ldots$ & 5.0 & 18.8 & 12.3 \\
\hline Project & $\ldots$ & $\ldots$ & $\ldots$ & & $\ldots$ & \\
\hline Nonproject & $\ldots$ & $\ldots$ & $\ldots$ & 5.0 & 18.8 & 12.3 \\
\hline Disbursements from IMF 7/ & $\cdots$ & $\cdots$ & $\cdots$ & 1.7 & 1.7 & 0.8 \\
\hline Exceptional financing (assumed) & $\ldots$ & $\ldots$ & $\ldots$ & 11.4 & 8.2 & 6.4 \\
\hline Available financing & 371.5 & 302.3 & 311.6 & 416.7 & 426.5 & 387.7 \\
\hline Residual financing gap 8/ & $\ldots$ & $\ldots$ & $\ldots$ & $\ldots$ & $\ldots$ & $\ldots$ \\
\hline
\end{tabular}

Sources: Rwandese authorities; and staff estimates and projections.

1/ Excludes budgetary and HIPC Initiative grants already disbursed, and humanitarian and technical assistance.

2/ Excludes project grants and loans and budgetary loans that have already been disbursed. Includes errors

and omissions for past years.

3/ Includes disbursed budgetary grants to the central government (including HIPC Initiative grants) as well as grants in the form of humanitarian and technical assistance. Budgetary grants (including HIPC Initiative grants) not yet disbursed are listed under the expected financial support. Amounts are shown net of outflowing public transfers.

4/ Budgetary loans not yet disbursed are listed under the expected financial support.

5/ In 2003, it reflects the debt rescheduling agreement with the OPEC Fund.

6/ All project grants and loans are not programmed financing and are therefore recorded under existing commitments.

7/ Includes expected Fund disbursements under the current PRGF arrangement.

8/ A negative sign implies a financing gap. 
Table 6. Rwanda: External Public Debt and Debt Service, 2001-06

(In millions of U.S. dollars, unless otherwise indicated; end of period)

\begin{tabular}{|c|c|c|c|c|c|c|}
\hline & 2001 & 2002 & 2003 & $\begin{array}{l}2004 \\
\text { Proj. }\end{array}$ & $\begin{array}{l}2005 \\
\text { Proj. }\end{array}$ & $\begin{array}{l}2006 \\
\text { Proj. }\end{array}$ \\
\hline Total external debt outstanding $1 /$ & $1,336.1$ & $1,401.0$ & $1,432.8$ & $1,485.5$ & $1,556.2$ & $1,510.0$ \\
\hline Multilateral & $1,185.2$ & $1,249.0$ & $1,273.6$ & $1,338.2$ & $1,414.9$ & $1,373.1$ \\
\hline \multirow{2}{*}{$\begin{array}{r}\text { Of which: IDA } \\
\text { IMF }\end{array}$} & 767.4 & 833.9 & 847.3 & 917.4 & 989.7 & 973.8 \\
\hline & 84.3 & 84.9 & 91.9 & 87.5 & 79.5 & 65.1 \\
\hline Bilateral & 150.4 & 151.5 & 158.6 & 146.8 & 140.7 & 136.3 \\
\hline Paris Club 2/ & 74.1 & 75.5 & 76.7 & 73.2 & 70.0 & 68.1 \\
\hline Non-P aris Club & 76.3 & 76.0 & 81.8 & 73.6 & 70.7 & 68.2 \\
\hline Commercial 3/ & 0.5 & 0.6 & 0.6 & 0.6 & 0.6 & 0.6 \\
\hline Debt service after bilateral rescheduling 4/ & 38.6 & 43.8 & 36.2 & 40.7 & 48.1 & 55.2 \\
\hline Principal & 27.3 & 32.3 & 24.0 & 28.7 & 35.6 & 43.0 \\
\hline Interest & 11.3 & 11.5 & 12.2 & 12.0 & 12.5 & 12.2 \\
\hline HIPC Initiative assistance & 23.9 & 25.6 & 23.3 & 27.5 & 36.0 & 43.3 \\
\hline Multilateral & 23.9 & 25.6 & 23.0 & 27.2 & 32.0 & 38.8 \\
\hline Bilateral 5/ & 0.0 & 0.0 & 0.3 & 0.3 & 4.0 & 4.5 \\
\hline Commercial & 0.0 & 0.0 & 0.0 & 0.0 & 0.0 & 0.0 \\
\hline Debt Service after HIPC Initiative assistance 4/ & 14.7 & 18.2 & 12.9 & 13.2 & 20.2 & 18.4 \\
\hline Principal & 10.4 & 13.4 & 8.6 & 9.3 & 15.0 & 14.3 \\
\hline Interest & 4.3 & 4.8 & 4.4 & 3.9 & 5.3 & 4.0 \\
\hline \multicolumn{7}{|l|}{ Memorandum items: } \\
\hline Total debt stock (in percent of GDP) & 78.4 & 80.9 & 85.1 & 86.6 & 85.7 & 78.5 \\
\hline \multicolumn{7}{|l|}{ Debt service after bilateral rescheduling; in percent of: } \\
\hline Exports of goods and services & 24.6 & 33.0 & 28.6 & 29.4 & 30.1 & 31.6 \\
\hline Government revenue $6 /$ & 19.8 & 20.7 & 15.9 & 17.6 & 19.6 & 21.1 \\
\hline Government current expenditure and net lending & 10.8 & 11.9 & 8.9 & 8.4 & 10.1 & 11.4 \\
\hline \multicolumn{7}{|l|}{ Debt service after HIPC Initiative; in percent of: } \\
\hline Exports of goods and services & 9.3 & 13.8 & 10.3 & 9.6 & 12.7 & 10.5 \\
\hline Government revenue 6 / & 7.5 & 8.6 & 5.7 & 5.7 & 8.2 & 7.0 \\
\hline Government current expenditure and net lending & 4.1 & 4.9 & 3.2 & 2.7 & 4.3 & 3.8 \\
\hline Net present value (NPV) of debt (in US\$ million) 7/ & 330.6 & 385.2 & 415.8 & 468.5 & 531.3 & 580.2 \\
\hline NPV of debt-to-exports ratio $7 / 8 /$ & 236.2 & 263.0 & 299.9 & 354.5 & 376.4 & 368.6 \\
\hline
\end{tabular}

Sources: Rwandese authorities; and Fund staff estimates and projections.

1/ After rescheduling, including arrears and new debt (the latter includes assumed project disbursements for the perioc Data are preliminary, pending a full debt sustainability analysis, and do not reflect changes in exchange rates.

2/ All Paris Club debt is pre-cut-off date debt.

3/ Refers to loans by commerical creditors which were guaranteed by Paris Club creditors.

4/ Including Fund.

5/ Reflects impact of topping up to Cologne terms for non-ODA debt of Paris Club creditors from 2003. During 200 the data include the effect of a stock-of-debt operation at the completion point on debt service.

6/ Excluding grants.

7/ Based on assumptions about expected new borrowing. For illustrative purposes, the numbers are shown as if HIPC had been delivered unconditionally as of 1999 (data available at the time of the decision point).

Data are preliminary, pending a full debt sustainability analysis.

8/ The exports denominator is calculated using a three-year backward-looking average.

\section{(C) International Monetary Fund. Not for Redistribution}


Mrs. Anne O. Krueger

Deputy Managing Director

International Monetary Fund

Washington, D.C. 20431

U.S.A.

Dear Mrs. Krueger,

Ten years after genocide, Rwanda has established strong democratic institutions with the adoption of a new constitution approved by referendum and the conduct of presidential and legislative elections. Government therefore believes that with these firm democratic institutions in place, Rwanda has created a strong foundation for a new future for full implementation of its poverty reduction strategy.

Our economic strategy in the medium-term is therefore in line with our policies and priorities set out in Vision 2020. These have been articulated in the participatory PRSP and subsequent sectoral strategies and also reflect the aspirations of the nation. In line with our economic strategy, we intend to implement strong policies that will raise agricultural productivity and export base to achieve external sustainability. We believe these are essential for wide ranging and sustainable poverty reduction.

In support of our political, economic and social programs, Government reaffirms its commitment to strengthen the foundation for regional peace and security in the Great Lakes area.

The attached memorandum of economic and financial policies (MEFP) reviews the implementation of the 2003 program and sets out the objectives and policies that the government intends to pursue in 2004.

In light of the progress achieved in the implementation of the program for 2003, and given the supporting details provided in the MEFP, the Government of Rwanda requests a waiver for the missed observance of the performance criteria for end-June 2003 on the domestic fiscal balance, the net accumulation of domestic arrears, and the contracting of nonconcessional external loans; for end-December 2003 on the net foreign assets of the National Bank of Rwanda, reserve money, net banking system credit to government, the domestic fiscal balance, the net accumulation of domestic arrears, and the contracting of nonconcessional external loans; and for the structural performance criteria on the submission of a revised investment code, the issuance of financial instructions, and the incorporation of tax incentives into the structure of the income tax. The Government also requests a third disbursement under its PRGF arrangement with the Fund in an amount equivalent to SDR 1.142 million, following completion of the second and third reviews by the Fund's 
Executive Board. In view of the delay of the completion point under the enhanced HIPC Initiative compared to the original schedule of December 2002, the government requests additional interim HIPC Initiative assistance in an amount of SDR 4.482 million for the period from June 9, 2004 to June 8, 2005.

The Government of Rwanda will continue to provide the IMF with such information as the Fund requires to assess Rwanda's progress in implementing the policies described in this letter and the accompanying MEFP. In addition, the Government will continue to consult with the Fund on its economic and financial policies, in accord with the Fund's policies and practices on such consultations.

Yours sincerely,

\author{
/s / \\ François Kanimba \\ Governor \\ National Bank of Rwanda
}

\author{
$/ \mathrm{s} /$ \\ Donald Kaberuka \\ Minister of Finance and \\ Economic Planning
}

Attachments: Memorandum of Economic and Financial Policies

Technical Memorandum of Understanding 


\section{Memorandum of Economic and Financial Policies of the Government of Rwanda for 2004}

\section{Performance Under the 2003 Program}

1. Macroeconomic performance during 2003, under the second annual PRGF arrangement, was greatly affected by poor weather and by unprogrammed spending related to the challenge of assuring a smooth political transition. Nonetheless, with strengthened economic institutions and financial management and oversight, and solid achievements in the implementation of poverty programs, including the achievement of a substantially higher primary school enrollment rate and a reduced incidence of communicable diseases, the foundation has been set for rapid progress in 2004, under the poverty reduction and growth strategy.

2. The rate of real GDP growth fell below the targeted range in 2003, as poor rains led to a fall in agricultural output. In turn, as a result of sharp increases in food prices, along with rising import prices, inflation edged up to 7.7 percent at end-2003, compared with the program target of 3 percent. As grant financing for the political transition and health programs did not materialize and, with other external assistance disbursements delayed to January 2004, international reserves fell by US\$29 million, to the equivalent of 5.0 months of import cover.

3. The nonobservance of quantitative performance criteria for end-December 2003 resulted from overspending elections, the financing of a hotel project, and delayed donor disbursements of external program assistance. Action is being taken to correct these slippages in 2004. These include a substantial reduction in net credit to government from the banking system, slower reserve money growth, and a fiscal program that incorporates contingent cuts to assure that the domestic fiscal balance remains consistent with macroeconomic objectives. The missed performance criteria on new non-concessional external debt resulted from an energy rehabilitation loan with a grant element marginally below the 50 percent floor set in the program. The financing for this project will be brought to the required concessionality level by end-June 2004.

4. On the structural side, the structural performance criteria for submission of a revised investment code, issuance of financial instructions for more effective expenditure management, and incorporation of tax incentives into the structure of the income tax were not observed as a result of human capacity constraints. Firm dates have been set for the completion of the missed criteria and action is being taken to assure their realization. Three prior actions, now being implemented, further underscore the strength of resolve on the advancement of the structural agenda,

5. Some progress was achieved toward the fiscal objectives under the 2003 program. Domestic revenue collections, equivalent to 13.5 percent of GDP modestly exceeded the projected level, as weaker-than-expected excise taxes were offset by improved direct tax performance, largely due to changes in the tax on professional remuneration. Nontax revenue 
was 0.1 percentage points of GDP above the projected level, as improved collection efficiency resulting from the transfer of administrative fees collection to the Rwanda Revenue Authority (RRA) offset disappointing dividend income from public enterprises.

6. Domestic spending exceeded the program target by 1.2 percent of GDP, largely as a result of spending on elections and, late in the year, on unanticipated goods and service outlays. Nonetheless, the performance criteria for recurrent priority spending were met and recurrent defense spending was held to 2.7 percent of GDP, compared with 2.9 percent in 2002, and down from 3.3 percent in 2001. Expenditure on the constitutional referendum in May, and on presidential and legislative elections during August-September 2003, equivalent to 1.6 percent of GDP was roughly twice the budgeted amount. Overall, exceptional spending exceeded the (adjusted) indicative program target by 0.5 percent of GDP. Partly offsetting the impact of this higher spending, savings equivalent to 0.1 percent of GDP were realized on domestically financed capital spending, and outlays for the strategic petroleum reserve were more than halved. Finally, despite progress towards regional peace, voluntary participation in the demobilization program fell short of projections and 0.6 percent of GDP in related outlays were deferred to 2004. Largely as a result of startup delays for grant-financed road works, preliminary data indicate that foreign-financed development expenditures for 2003 were equivalent to about 4.2 percent of GDP -2 percentage points below the programmed level.

7. Given this, the domestic fiscal deficit amounted to RF 50 billion (5.5 percent of GDP) in 2003, exceeding the (adjusted) ceiling set as a performance criterion by RF 26.7 billion. The overall deficit on central government operations amounted to 10.5 percent of GDP before grants, and 2.5 percent of GDP after grants. While the settlement of pre-2003 domestic arrears exceeded the programmed value for end-December 2003 only marginally (by 0.1 percentage points of GDP), the performance criterion on the net reduction of domestic arrears was missed by 1.7 percent of GDP, as the stock of bills payable for 2003 increased substantially because government payments were slowed in light of expansionary monetary conditions.

8. The implementation of monetary policy during 2003 was complicated by the reliance on central bank credit to cover the cost of higher-than-programmed election spending that emerged beginning in July 2003. Additional factors adding to the liquidity expansion were the unprogrammed domestic financing of a hotel project, the relaxation of the limit on the net foreign exchange positions of commercial banks, and lending to the economy by nonbank financial institutions, following the recapitalization of the UBPR credit union under a World Bank loan.

9. As the annual growth rate of broad money (at current exchange rates) rose from 15.8 percent in June 2003 to a peak of 19.0 percent in September (the September reserve money benchmark was missed by 8.2 percent), the National Bank of Rwanda (NBR) took corrective measures. It raised the discount rate from 13.5 percent to 14.5 percent in August and withdrew liquidity from the system through open market operations and higher foreign exchange sales to the market. Consequently, the interest rates on treasury bills and the central 
bank intervention rate rose from about 11 percent in mid-September to about 13 percent in mid-November. However, the central bank was unable to fully sterilize the fiscal impulse, and reserve money exceeded the performance criterion that had been set for end-2003 by 9.6 percent.

10. Largely as a result of shortfalls and delays in external assistance inflows, the net foreign assets of the NBR declined to RF 57.8 billion at end-2003, from RF 72.6 billion at end-2002, falling short of the (adjusted) floor set under the program by RF 27.8 billion. ${ }^{8}$ Net credit to government from the banking system increased to RF $171 \frac{1}{2}$ billion during 2003 , exceeding the (adjusted) program ceiling by RF 32.6 billion. In addition, banking system credit to the economy grew by 14.8 percent -7 percentage points above the targeted level. As a result, broad money grew by 12.2 percent (on an annual basis) during 2003 4.2 percentage points above the targeted rate (evaluated at the program exchange rate).

11. The higher-than-programmed expansion in monetary aggregates, along with weak export performance (see below) contributed to growing pressure on the foreign exchange market during the second half of 2003. The Rwanda franc/U.S. dollar exchange rate, which had depreciated by 4.8 percent during the first half of 2003 , weakened by 8.2 percent during the second half of the year, despite an increase in the amount of foreign exchange offered by the NBR in its weekly auctions from US\$1.3 million to US\$1.8 million on average.

12. External sector performance deteriorated in 2003, reflecting the collapse of the international coltan market, weak international markets for coffee and tea, and slow progress towards improving productivity and establishing niche markets. While merchandise exports fell from US\$67 million in 2002 to US\$63 million in 2003, imports of goods and services increased moderately, causing a further weakening of the current account balance by 3.2 percentage points to 19.8 percent of GDP. External budgetary assistance inflows fell short of the projected value by US $\$ 73$ million (4.4 percent of GDP), as projected World Bank and African Development Bank loans, and European Union grant disbursements were delayed, and additional grant financing to cover election costs and one-off health expenditures, which had been projected at US\$18 million, amounted to only US\$6 million. Given this lower-than-anticipated external budgetary assistance, the NBR reduced its net foreign assets to US\$113 million at end-December 2003, a decline of US\$30 million relative to end-2002.

13. In February 2003, a loan agreement was signed with the OPEC Fund and Arab Bank for African Development (BADEA) as part of a financing package for an energy sector project. While the grant element of this loan was substantial (47 percent), it did not meet the concessionality test set under the PRGF arrangement and, as a result, the performance criteria for new nonconcessional external debt for end-June and end-December 2003 and the corresponding benchmark for end-September 2003 were not observed. Agreements have

\footnotetext{
${ }^{8}$ Evaluated at the program exchange rate of RF 511.9=US\$1.
} 
been reached that will increase the grant element of the loan and bring the terms within the required concessionality threshold. In this regard, ratification by BADEA's Board of Directors is scheduled for June 9, 2004. Government guarantees issued in August 2003 for an external US\$10 million loan on commercial terms, as part of the financing package for a major hotel project will be repaid in full by end-May, 2004. As no new nonreschedulable arrears have been accumulated in 2003, the performance criterion for external arrears was met. To strengthen external debt service management, an action plan is currently being implemented. Furthermore, the second phase of a capacity building program with Debt Relief International, was started in January 2003. Activities so far undertaken have included three country missions to deal with institutional reforms as well as the organization of a workshop on Rwanda's longer-term debt sustainability.

14. Rwanda's external debt amounted to US\$1.4 billion (85 percent of GDP) at end-2003. Given the heavy debt service burden associated with this stock, Rwanda continues to depend on interim debt relief from its creditors. In this regard, Paris Club creditors, in June 2003, decided to extend the consolidation period from June 20, 2003 to June 30, 2004 on the basis of Cologne terms. Paris Club creditors have been requested to further extend the consolidation to end-June 2005. During 2003, Rwanda reached agreements on debt cancellation or rescheduling with some of its Paris Club creditors; remaining negotiations are expected to be concluded prior to the June 30, 2004 deadline for reaching bilateral agreements. Rwanda is also making efforts to reach rescheduling agreements with its nonParis Club creditors. An agreement with the Kuwait Fund was signed on February 5, 2003, rescheduling the stock of debt including arrears.

15. The agenda for structural reform in 2003 included steps to improve the efficiency and productivity of the private sector, and to strengthen the effectiveness, comprehensiveness and accountability of public finances and the financial sector. Substantial progress was achieved in each of these areas. In a step to strengthen Rwanda's public utilities, a five-year management contract for the electricity and gas parastatal, Electrogaz, was agreed in March 2003 and entered into operation in October, 2003. In telecommunications, the offer for sale of the public telecommunications company, Rwandatel, will be issued in May 2004. As a critical component of the export promotion strategy set out in the PRSP, the government sold 13.4 percent of its shares in Sorwathe (a tea processing factory) to a private investor and a further 10 percent to a farmer's association. In addition, two state-owned tea estates were offered for sale at end-August 2003, following the adoption of enabling legislation by the National Assembly. One of the bids received in early-February 2004 remains under review, with a decision expected shortly, while the other bid has been accepted. The privatization of three rice processors, and mining, printing, hotel, and livestock enterprises will constitute the next steps in this area.

16. Similarly, important steps were taken towards addressing structural issues in the financial sector. The framework for the supervision of microfinance institutions was strengthened, with on-site inspections slated to begin in 2004. In addition, during 2003, loan recovery improved, access to mortgage finance was widened, and information sharing was initiated between banks on creditor risk. With this, the share of gross nonperforming loans in 
total loans stabilized at 37.4 percent at end-December 2003. A World-Bank-sponsored financial sector study was completed in April 2004. In addition, the restructuring of the Caisse Hypothécaire du Rwanda (CHR) is planned.

17. While the tender for sale of the Banque Commerciale du Rwanda (BCR, a structural benchmark for end-June 2003) was delayed, substantial progress was made towards the sale of the bank. An action plan was implemented for improving BCR's profitability ahead of privatization. The bank was advertised for sale in major publications in June 2003, and prospective investors subsequently undertook due diligence inspections. Under a revised timetable, the offer for sale of the bank is expected to take place by end-July 2004.

18. The strengthening of public financial management constituted the most ambitious component of the reform agenda. While the achievement of specific objectives has proven to be a greater challenge than had been anticipated, overall progress can only be rated as a major success. The adoption of a new constitution at end-May 2003 established a new and clear framework for the institutions of public finance. The Auditor General (AG) was vested with sole accounting authority, reporting directly to parliament. During 2003, the AG's office audited the 2002 accounts of 41 public entities, including nine ministries (including the Ministries of Defense and Finance), with a report issued to Parliament in March 2004. The framework for government financial operations was clearly set out. With this, and with technical assistance from the Fund staff, a new Organic Budget Law (OBL) was drafted and readied for submission to parliament, planned for June 2004. (The submission of the OBL to parliament has been delayed from the September 2003 program benchmark to end-June 2004. As a result of the delayed submission of the OBL, the issuance of financial instructions, which will provide complementary operational details and which had been set a structural performance criterion for end-January 2004, is now set for end-May 2004.)

19. Following a joint World Bank/United Kingdom financial accountability review and assessment plan (FARAP) diagnostic mission in February 2003, a draft action plan was issued in June 2003. This action plan, which incorporates inputs from the Fund, builds on existing commitments and previous advances, covering the legal and institutional framework and budget and expenditure management. At the same time, a number of the structural actions envisaged under the 2003 program were realized, including a comprehensive review of tax exonerations, exemptions and incentives. (The respective structural performance criterion was completed by end-June 2003.) Progress was also made on structural benchmarks set under the program: a draft report on the implementation of the 2002 development budget was issued in July 2003, and finalized the following September; a list of overdue obligations scheduled for clearance during 2003 was issued in September 2003; and, with Fund staff support, drafting of a new investment code and tax laws was initiated.

20. Despite the substantial achievements noted above, the calendar set for reforms in the area of public financial management proved overly ambitious. In addition to the previously noted delays on the OBL and supporting financial instructions, the preparation of legislation for submission of a revised investment code to parliament, incorporating tax incentives into the tax law (a structural performance criterion), could not be achieved under the timetable 
fixed under the 2003 program. These actions, as noted in paragraph 18, will be realized over the coming months. Substantial progress was made in implementing other structural benchmarks including, setting the Ministry of Finance and Economic Planning's (MOF) internal audit department fully in place (a structural benchmark); the development of a monthly reporting mechanism for district governments, and the closure of government dormant accounts.

21. The National Bank of Rwanda (NBR) made substantial progress towards meeting the structural benchmarks set in the reform agenda for the financial system. In an effort to improve regulatory compliance, the NBR issued new instructions on solvency ratios, risk exposure and provisioning at end-December 2003. Actions taken in response to the recommendations of the April 2003 Fund safeguards mission are detailed in paragraphs 4950. In addition, the central bank continued its consultations on the drafting of new antimoney laundering legislation. Related to this, Fund and World Bank staff will provide technical assistance in drawing up required legislation, and an enforcement unit will be established at the NBR during 2004.

\section{The MEDIUM-Term STRATEgY}

22. Rwanda's medium term strategy for poverty reduction and economic growth continues to be guided by the policies set out in its Poverty Reduction Strategy Paper (PRSP), which was issued in June 2002. The PRSP progress report issued in July 2003 sets out the substantial advances made in human resource development as well as the contributions made by improved governance and regional peace to development objectives. Looking forward, the immediate challenge will be the full elaboration of policy frameworks in the nonsocial sectors and their implementation. In particular, in the period ahead, an increased focus will be placed on improving the supply response of the economy, reducing its vulnerability to exogenous shocks, and strengthening the performance of the external sector. This approach will include programs to improve access to fertilizers and improved seeds, and rural infrastructure development, with a view to increasing employment and incomes in rural areas. The export promotion strategy, currently under review, aims at the diversification of the export base through the privatization of the tea sector, a shift in coffee production to the specialty market, and the development of tourism activities.

23. The medium term strategy recognizes the critical importance of macroeconomic stability in supporting the attainment of poverty reduction objectives and envisages, over the medium to long term, the gradual reduction of macroeconomic imbalances and dependence on external grants and borrowing. The phasing out of transitional programs and targeted improvements in domestic resource mobilization, and the impact of the export promotion strategy, combined with prudent macroeconomic policies, will contribute to the realization of this objective. Furthermore, at least in the short run, a strong effort to mobilize more grants from development partners, to supplement current medium and long term grant commitments, will help to assure that external sustainability is achieved and maintained. Under a policy cooperation agreement that was reached with cooperating partners in November 2003, communications on external budgetary assistance will be moved forward in 
the planning cycle, donors will aim towards multi-year commitments, and policies will be drawn from a commonly supported framework with joint progress reviews.

\section{The Economic Program for 2004}

24. The program for 2004 will provide resources to assure that substantial progress is made towards achieving poverty reduction objectives and for placing Rwanda on a trajectory towards achievement of the millennium development goals by 2015. This agenda will include, beginning in 2004, a 10 percent rise in the number of educators, with fees eliminated for primary education. Funding for health programs has been substantially increased. At the same time, substantial focus will be placed on strengthening export performance, moving forward with the privatization agenda, making the legislative and regulatory climate more conducive to investment, and improving economic infrastructure and productivity. This will continue to be supported by steps to strengthen management, transparency and accountability in the financial sector and in public finances.

25. Based on the PRSP, the medium-term macroeconomic objectives are to (i) achieve annual real GDP growth of at least 6 percent; (ii) limit end-period inflation at 5 percent in 2004 and 3 to 4 percent thereafter; and (iii) reconstitute and maintain gross international reserves equivalent to at least 6 months of imports. As Rwanda's population growth rate is about 3 percent, these objectives should yield significant increases in per capita real income over the medium term.

\section{A. Macroeconomic Policies}

\section{Fiscal policy}

26. The fiscal program for 2004 aims to consolidate gains made in revenue policy and administration over the preceding two years, while drawing on substantial increases in pledged external grant assistance to finance an ambitious, yet well-focused, government expenditure program. While no major tax policy changes are planned for 2004, past reforms will come fully into play. Custom duties on a reciprocal basis with other FTA members were eliminated as a result of Rwanda's entrance into the COMESA free trade area (FTA) on January 1,2004. Direct tax income will reflect on a full year basis the impact of revisions in marginal rates and in the taxation of professional remuneration. In addition, further action to strengthen revenue administration, building on technical assistance from the IMF and United Kingdom, including the formation of a large taxpayer unit at the Rwanda Revenue Authority, will support strong revenue performance. All told, government domestic revenue is projected to steady at 13.5 percent of GDP in 2004 .

27. Government domestic spending is budgeted to increase to the equivalent of 21.3 percent of GDP in 2004, which reflects a substantial increase from the $143 / 4$ percent that had been envisaged under the initial PRGF arrangement. As previously noted, given increased availability of external financing, implementation will begin in earnest on a full range of programs, including new constitutionally mandated institutions, free basic 
education, government counterpart contributions for a comprehensive HIV/AIDS treatment program, supplementary pay for health sector workers, human resource development in tertiary education, labor intensive rural works, and export promotion, all of which are drawn from the PRSP. The 2004 budget also reflects 0.5 percentage points of GDP in demobilization outlays that have been carried over from 2003. As a result, government priority spending is budgeted to rise to 45 percent of domestic expenditure, as targeted. Beyond this, net lending will increase by the equivalent of 1.5 percentage points of GDP to cover guarantees issued to finance hotel construction in 2003 and to finance completion of the hotel project in 2004, and to the electricity parastatal (Electrogaz) to finance the procurement of emergency generating equipment and related fuel oil.

28. As a result, the domestic fiscal deficit under the 2004 budget is set to rise to the equivalent of 7.8 percent of GDP. The budget also provides for the settlement of 0.9 percent of GDP in unpaid government bills (on a net basis) that were outstanding at end-2003 and outlays equivalent to 0.7 percent of GDP on government domestic arrears accumulated prior to 2002. Taken together with payments to nonbank public entities, the total budgeted government borrowing requirement for domestic operations (net of taxes) amounts to about 10.3 percent of GDP.

29. Currently identified external budgetary grants (equivalent to 8 percent of GDP) fall short of fully meeting financing requirements, As additional external borrowing to meet the financing gap would raise Rwanda's net present value of external debt-to-export ratio further above established norms, and as rapid spending could add to the substantial monetary overhang at the beginning of 2004, budgetary operations equivalent to 1.4 percent of GDP were rephased from the first to the second half of 2004. These operations will be authorized only to the extent that additional external grant financing is secured and if monetary policy remains on track.

\section{Monetary policy}

30. Monetary policy has been set with the objective of bringing inflation down to 5 percent at end-2004. Accordingly, and taking into account the GDP growth objective for the monetized economy, broad money is targeted to increase by 11 percent (at the program exchange rate) by year-end. Targets for the expansion of the monetary base have been set accordingly. The NBR will closely coordinate with the Ministry of Finance through the recently established Treasury Committee, in order to ensure that fiscal and monetary policy implementation operate effectively. The development of a secondary market for government securities, which is now underway, is expected to strengthen the effectiveness of the NBR's policy implementation in the period ahead. In order to improve the information base for monetary control, the NBR will start to publish a new consumer price index for Kigali during the first half of 2004.

31. Despite the projected increase in external assistance inflows of grants and loans, given increased foreign exchange sales and government external payments, the NBR's international reserves will fall by US\$6 million and, with this, gross official reserves will fall 
to the equivalent of 4.6 months of import cover. Net credit to government by the banking system is targeted to increase by RF 5.5 billion, while credit to the economy is programmed to grow by 10.6 percent.

32. Regarding the NBR's foreign exchange transactions, the operation of the auction will be further strengthened. The NBR will review, with assistance from the IMF staff, the current auction system, in order to limit collusion among auction participants and ensure that auction exchange rates are market determined.

\section{External sector}

33. The deficit on the external current account, excluding current official transfers, is expected to rise to 21.4 percent of GDP in 2004, from 19.8 percent in 2003. Underlying this projection, merchandise exports appear set to increase modestly to US\$68 million, mainly as a result of an increase in the volume of tea and nontraditional exports. Gains in coffee exports, while benefiting from a modest increase in international prices, are likely to be constrained by poor rains, shortages of inputs, and limited infrastructure. The international sale of fully washed coffee is likely to remain relatively flat, while coltan export volumes are projected to continue their secular decline in 2004. Merchandise imports, driven by increased outlays for the development budget and intermediate goods, including fertilizers, along with the previously noted purchase of electricity generating equipment and fuel oil, are projected to rise by 16 percent.

34. As part of efforts to address the structural issues underlying Rwanda's disappointing export performance, an export promotion commission, established in December 2003, submitted a preliminary short-term action plan for cabinet consideration in February 2004. That plan incorporates a working capital facility for the coffee sector, funds for producer and grower-targeted programs, and an export finance facility. To ensure the compatibility of these priority actions with the government's broad development objectives as well as their swift implementation, external partners have been asked to provide support in the articulation of necessary policies and institutions. Beyond this, work continues on a strategy for the diversification of the export base, which will be facilitated by the return to stability in the Great Lakes region, and a growing outreach to COMESA markets.

35. The decline in Rwanda's exports of goods and services in 2003 further weakened the already fragile debt sustainability indicators. Given this, and taking into account the importance of reaching the Completion Point (CP) under the HIPC Initiative as soon as possible, action is continuing in order to meet the remaining floating CP triggers, while maintaining a prudent debt management policy. New debt will only be contracted on highly concessional terms, and will be limited to levels consistent with assumptions made at the time of the Decision Point. In this context, the government reserves the option to rephase the execution of its development budget in case the programmed external grant financing were only partially forthcoming. Efforts to reach bilateral debt rescheduling agreements with Paris Club creditors on Cologne terms, and to regularize relations with all external creditors through the signing of rescheduling agreements on terms comparable to those provided by 
the Paris Club, are currently underway. We will also strengthen efforts to ensure the participation of all creditors in the HIPC Initiative.

\section{B. Structural Policies}

\section{Steps to improve economic productivity and external sustainability}

36. Government activities to strengthen Rwanda's economic productivity and external sustainability will be significantly intensified in 2004 . The privatization of the telecommunications parastatal (Rwandatel), along with the finalization of the sale of tea factories at Mulindi and Pfunda, and the preparation for the offering of the seven remaining estates, will contribute to this effort. In addition, a comprehensive policy framework for Rwanda's water sector and energy regulation is slated for development, with World Bank assistance. The government will continue efforts to sell a majority share in Prime Holdings, which developed two hotel projects during 2003, to private investors. The government is also continuing discussions with private investors on the development of a methane gas project at Lake Kivu.

37. At the same time, additional steps will be taken to further strengthen the legal framework, in order to create an attractive environment for investment. In particular, a new investment code has been drafted and will be submitted to parliament by end-September 2004. Moreover, a new mining code is being drafted and will be submitted for parliamentary consideration by end-2004.

38. Given the disappointing economic performance of 2003, the implementation of the development strategy set out in the PRSP will be accelerated. A labor intensive public works project, developed with donor assistance, will initiate operations, including land terracing, reforestation, wetlands reclamation, road rehabilitation and extension, and the construction of district markets. Alongside this, the Ministry of Agriculture, in consultation with supporting partners, is reassessing its policy framework and reviewing modalities for improving access to agricultural credit, and the distribution of fertilizer and improved seeds.

39. In the external sector, the government export promotion taskforce that was constituted in December 2003 will submit a detailed export promotion action plan for cabinet consideration by end-June 2004. The plan will include recommendations for the establishment of an effective management structure and will set out a specific and timebound agenda for strengthening export performance.

\section{Extending reforms in the legal framework and institutions}

40. The important achievements realized during 2002-03 in establishing modern, efficient and transparent laws and institutions for Rwanda's public finances will be extended in 2004. The new organic budget law, which is now pending cabinet review, will be submitted to parliament by end-June 2004. Supporting this, critical financial instructions have been readied and will be issued in tandem with the budget law. A draft income tax law, prepared with technical assistance from the IMF, eliminating ad hoc exemptions and 
exonerations in line with the recommendations of an IMF technical assistance report, was finalized and presented for cabinet consideration at end-March 2004. The submission of the new tax law to parliament is targeted to take place by June 30, 2004, and will be accompanied by a new procedures manual. The new procurement code, drafted in 2003, is slated for cabinet approval by end-May 2004. Finally, a draft customs law was finalized and sent for cabinet consideration at end-June 2004. It is expected that, with parliamentary approval, the law will take effect beginning with the 2005 budget.

\section{Strengthening the administration of public finances}

41. Building on earlier achievements and on a broad range of technical assistance from various development partners, including the IMF, a draft action plan, reflecting the findings of a financial accountability review and assessment, conducted by a United Kingdom-led team in cooperation with the World Bank, was issued in June 2003. In February 2004, a treasury management team was appointed to manage the plan's implementation, building on the findings of a European Union-sponsored team that visited Rwanda in March 2004 to assess associated costs. In tandem, the World Bank will conduct, in conjunction with the development of a new poverty reduction strategy credit, a country financial accountability assessment, and the Fund and the World Bank will jointly update their earlier HIPC assessment and action plan, providing a supporting review of government financial administration.

42. While the above noted reviews are in process, the implementation of the current agenda for the strengthening of public financial management will continue. For 2004, programmed actions will focus on budgeting, internal accounting, and decentralization. In particular, a new chart of government accounts for all units of government, developed in 2003 and tested, early in 2004, will be applied beginning with the 2005 budget. At the same time, with assistance from the IMF staff, the government's computerized fiscal operations reporting system will be extended to cover the provincial level, and the reporting mechanism extended to cover district government operations by end-December 2004, supporting enhanced monitoring, including monthly reporting.

43. Efforts to streamline the management of government bank accounts will continue. The task force established to implement this agenda will issue a progress report by endSeptember 2004. In addition, following the publication of required notifications, in 2004 the NBR will proceed with the closure of accounts which are either dormant or operating outside of controlling regulations, and will issue a progress report by end-December 2004. At the same time, the Ministry of Finance and Economic Planning intends to intensify efforts to reconcile on a monthly basis the balances on government budgetary operations with the movements in its financing balances. IMF staff will also provide assistance on treasury reforms, including the progressive phase out of nontreasury bank accounts, and the adoption of a treasury single account system, beginning in 2005. In this regard, monthly reconciliation statements, clearly showing the unreconciled differences between fiscal and monetary data, will be published on the Ministry's website on a quarterly basis, with no more than a one month lag, beginning in July 2004. 
44. In order to strengthen the budgeting process, an inventory of the physical assets of the central government line ministries, provinces, public enterprises and joint development projects is now scheduled for completion by end-December 2004 and will be annexed to the 2005 budget. In addition, reflecting a delay from last year, the 2005 budget will include a statement of tax expenditures associated with both existing and new policies included in the budget, a statement of assets and liabilities of all levels of government, financial statements of public enterprises, a statement of consolidated government equity holdings, a consolidated budget of the districts, a fiscal risk assessment, and a list of all contingent liabilities.

45. Substantial progress has been made in making government finances more transparent. The Auditor General's report on government 2002 operations will be made public at endJune 2004. In addition, in accordance with the law, consolidated central government accounts for 2003 were transmitted to the Auditor General at end-March 2004, and his audit based on those accounts will be submitted to parliament by end-June 2004. In 2004, as in 2003, the Auditor General will conduct audits of the 2003 operations of every central government ministry. As in 2003, a detailed list of pre-2002 domestic obligations scheduled for clearance during 2004 will be issued by end-June 2004 . $^{9}$

46. Efforts will continue to strengthen the Rwanda Revenue Authority (RRA). The reform agenda at the RRA in 2004 includes the establishment of a large taxpayer office and an operations policy department, along with the appointment of a single commissioner for tax operations. The system of tax identification numbers will be improved, accommodating the prompt elimination of separate value added tax registration numbers. Late filing penalties for tax returns will be reviewed and tax legislation will be updated to include penalties for tax fraud. The computerization of the tax (and customs) administration will continue during 2004.

\section{Continuing financial sector reforms}

47. The NBR will further increase its efforts to reestablish the observation of prudential regulations on a regular basis. In order to achieve this objective, it will work with commercial banks to agree on action plans, including specific steps, and deadlines and sanctions, for bringing them into compliance with banking regulations by end-December 2004. Agreement on the plans is targeted for end-June 2004. With regard to the regulation on the net open foreign exchange position, by May 31, 2004 the central bank will restore the governing regulation to the text in force one year earlier. In 2004, the central bank supervision department plans to conduct full annual audits of four commercial banks and the Rwandan Development Bank. The NBR plans to audit all commercial banks annually, beginning in 2005 .

\footnotetext{
${ }^{9}$ The list will set out amounts to be settled for salaries, goods and services (with details on payments to public utility companies, other public enterprises and the social security fund), and overdue payments including cumulating penalties for late payment (e.g., to road contractors). The list will indicate which of these payments have been authenticated by the Auditor General.
} 
48. The sale of Banque Commerciale du Rwanda (BCR), delayed from 2003 in order to accommodate due diligence requests from interested investors, is now planned to be completed by end-July 2004. The rehabilitation of CHR will be completed in 2004. Following the completion of the World Bank-sponsored financial sector study, a joint FundWorld Bank financial sector action plan (FSAP) mission has been scheduled for the fourth quarter of 2004. The FSAP will provide a comprehensive program for the reform and development of Rwanda's financial sector.

\section{Safeguards}

49. Key recommendations of an IMF safeguards assessment for the improvements of the NBR's control, accounting, reporting and auditing systems have been implemented. The recommendations of the safeguards assessment that have been implemented include conduct of external audits of the NBR for 2001 and 2002; the establishment of a formal policy for the conduct of annual external audits; the adoption of resolution to publish the audited financial statements within six months of the financial year; and the limitation of 2004 distributable profit to realized gains, net of unrealized losses.

50. Several recommendations by the IMF and external auditors are still in the process of implementation. IMF recommendations not yet (fully) implemented include the establishment of written procedures to ensure that monetary data used for program monitoring follow agreed definitions and are consistent with accounting records; an audit of program data as of September 30, 2003; the recruitment of additional accounting and internal audit staff; amendments to the central bank law with respect to internal accounting and audit procedures and the legal protection of senior staff in conducting their duties; and the tender for a study on potential improvements in the risk management of NBR.

\section{Program CoORdination ANd Monitoring}

51. The monitoring and program coordination is expected to be strengthened by the recently established treasury committee, which comprises members from the Ministry of Finance, the Rwanda Revenue Authority, and the NBR and is headed by the Secretary General of MOF. The program for 2004 will be monitored on a continuous basis with quantitative and structural performance criteria, benchmarks, and indicative targets. The fourth review will reflect performance on quantitative performance criteria for June 2004 and structural benchmarks and performance criteria through September 2004 and will be completed by mid-November 2004. The fifth review will reflect performance on quantitative performance criteria for end-December 2004, and structural benchmarks and performance criteria through end-March 2005, and will be completed by end-May 2005. A complete list of quantitative and structural performance criteria, as well as structural benchmarks, is included in Tables 1 and 2, respectively. The attached technical memorandum of understanding lays out the details of program design and terminology. 
Table 1. Rwanda: Quantitative Performance Criteria and Benchmarks 2003-04 (In billions of Rwanda francs, unless otherwise indicated)

\begin{tabular}{|c|c|c|c|c|c|c|c|c|}
\hline & \multicolumn{4}{|c|}{2003} & \multicolumn{4}{|c|}{$20041 /$} \\
\hline & Mar.* & Jun.** & Sep.* & Dec.** & Mar. & Jun.** & Sep.* & Dec.** \\
\hline & \multicolumn{8}{|c|}{ (Quantitative benchmarks*; and performance criteria on test dates**) } \\
\hline Net foreign assets of the NBR (floor on stock) $2 / 3 /$ & & & & & & & & \\
\hline Actual (program exchange rate) & 70.5 & 68.1 & 54.6 & 57.8 & $\ldots$ & $\ldots$ & $\ldots$ & $\ldots$ \\
\hline Adjusted program & 71.6 & 66.9 & 62.6 & 85.6 & $\ldots$ & $\ldots$ & $\ldots$ & $\ldots$ \\
\hline Program & 73.1 & 67.7 & 70.3 & 101.0 & 65.0 & 55.4 & 64.5 & 62.3 \\
\hline \multicolumn{9}{|l|}{ Reserve money (ceiling on stock) 4/ } \\
\hline Actual & 44.1 & 45.3 & 47.7 & 51.4 & $\ldots$ & & & $\ldots$ \\
\hline Program & 44.6 & 45.3 & 44.1 & 46.9 & 53.0 & 54.0 & 55.9 & 56.3 \\
\hline \multicolumn{9}{|c|}{ Net credit to the government by the banking system (ceiling on stock) $5 /$} \\
\hline Actual & 5.5 & 6.0 & 21.0 & 17.5 & $\ldots$ & & $\ldots$ & $\ldots$ \\
\hline Adjusted program & -0.1 & 8.6 & 5.3 & -15.1 & $\ldots$ & & & (2) \\
\hline Program & -0.1 & 7.7 & 0.1 & -27.4 & 24.4 & 25.0 & 18.1 & 22.8 \\
\hline \multicolumn{9}{|c|}{ Domestic fiscal balance (floor on cumulative flow since Dec. 31) 6/ } \\
\hline Actual & -7.4 & -18.0 & -36.8 & -50.2 & $\ldots$ & & $\ldots$ & $\ldots$ \\
\hline Adjusted program & 1.7 & -17.5 & -19.7 & -23.5 & $\ldots$ & $\ldots$ & $\ldots$ & $\ldots$ \\
\hline Program & -9.7 & -23.9 & -31.8 & -39.1 & -9.8 & -32.6 & -54.0 & -78.7 \\
\hline \multicolumn{9}{|c|}{ Recurrent priority spending (floor on cumulative flow since Dec. 31) 7/ } \\
\hline Actual & 12.9 & 30.6 & 44.8 & 59.1 & $\ldots$ & & & $\ldots$ \\
\hline Program & 12.5 & 30.3 & 42.6 & 56.2 & 14.9 & 37.5 & 62.5 & 88.2 \\
\hline \multicolumn{9}{|l|}{ New nonconcessional external debt (ceiling on flow) $8 / 9 /$} \\
\hline Actual & 5.0 & 5.0 & 5.0 & 5.0 & 3.0 & & & $\ldots$ \\
\hline Program & 0.0 & 0.0 & 0.0 & 0.0 & 3.0 & 0.0 & 0.0 & 0.0 \\
\hline \multicolumn{9}{|l|}{ Short-term external debt (ceiling on stock) $10 /$} \\
\hline Actual & 0.0 & 0.0 & 0.0 & 0.0 & $\ldots$ & $\ldots$ & $\ldots$ & $\ldots$ \\
\hline Program & 0.0 & 0.0 & 0.0 & 0.0 & 0.0 & 0.0 & 0.0 & 0.0 \\
\hline \multicolumn{9}{|c|}{ Stock of outstanding nonres chedulable external arrears (ceiling on stock) 11/ } \\
\hline Actual & 0.0 & 0.0 & 0.0 & 0.0 & $\ldots$ & . & & $\ldots$ \\
\hline Program & 0.0 & 0.0 & 0.0 & 0.0 & 0.0 & 0.0 & 0.0 & 0.0 \\
\hline \multicolumn{9}{|c|}{ Net accumulation of domestic arrears (ceiling on cumulative net accumulation since Dec. 31) } \\
\hline Actual & -6.6 & -9.0 & 1.6 & -1.0 & $\ldots$ & $\ldots$ & $\ldots$ & $\ldots$ \\
\hline Adjusted program & -5.9 & -9.7 & -21.1 & -16.7 & $\ldots$ & $\ldots$ & $\ldots$ & $\ldots$ \\
\hline \multirow[t]{2}{*}{ Program } & -5.9 & -8.9 & -8.9 & -7.9 & -11.2 & -13.0 & -14.7 & -17.0 \\
\hline & \multicolumn{8}{|c|}{ (Indicative targets) } \\
\hline Broad money (ceiling on stock) $12 / 13 /$ & & & & & & & & \\
\hline Actual & 143.4 & 144.7 & 155.5 & 162.7 & $\ldots$ & & & \\
\hline Program & 148.0 & 150.4 & 148.9 & 155.4 & 171.6 & 177.1 & 178.9 & 185.4 \\
\hline \multicolumn{9}{|l|}{ Extended Broad money (ceiling on stock) 12/14/ } \\
\hline Actual & $\ldots$ & $\ldots$ & $\ldots$ & $\ldots$ & $\ldots$ & $\ldots$ & $\ldots$ & $\ldots$ \\
\hline Program & $\ldots$ & $\ldots$ & $\ldots$ & $\ldots$ & 193.8 & 200.0 & 202.0 & 208.8 \\
\hline \multicolumn{9}{|c|}{ Exceptional spending (floor on cumulative flow since Dec. 31) } \\
\hline Actual & 6.7 & 13.7 & 30.4 & 40.2 & $\ldots$ & $\ldots$ & $\ldots$ & $\ldots$ \\
\hline Adjusted program & 8.1 & 13.7 & 24.7 & 35.4 & $\ldots$ & $\ldots$ & $\ldots$ & $\ldots$ \\
\hline Program & 9.8 & 17.6 & 30.7 & 41.8 & $\ldots$ & $\ldots$ & $\ldots$ & $\ldots$ \\
\hline & & & & emorand & ems) & & & \\
\hline Demobilization and reintegration expenditure & & & & & & & & \\
\hline Actual & 2.4 & 3.8 & 4.6 & 7.3 & $\ldots$ & $\ldots$ & $\ldots$ & $\ldots$ \\
\hline Expected & 4.1 & 7.7 & 10.6 & 13.7 & 3.5 & 7.5 & 9.9 & 12.6 \\
\hline Gross accumulated bills payable & & & & & & & & \\
\hline Actual & $\ldots$ & 2.3 & 13.7 & 11.3 & $\ldots$ & $\ldots$ & $\ldots$ & $\ldots$ \\
\hline Expected & $\cdots$ & 1.5 & 1.5 & 2.5 & 0.8 & 1.0 & 1.5 & 2.0 \\
\hline General budget support (in US\$ million) & & & & & & & & \\
\hline Received & 31.4 & 51.9 & 59.4 & 100.5 & $\ldots$ & & & $\ldots$ \\
\hline Expected & 34.2 & 57.2 & 83.3 & 161.9 & 35.1 & 90.1 & 149.4 & 201.0 \\
\hline Of which: budget support grants (expected) & $\ldots$ & $\ldots$ & $\ldots$ & $\ldots$ & 35.1 & 70.1 & 109.4 & 156.0 \\
\hline Earmarked budget support (in US\$ million) & & & & & & & & \\
\hline Received & $\ldots$ & 0.7 & 3.6 & 5.8 & $\ldots$ & $\ldots$ & $\ldots$ & $\ldots$ \\
\hline Expected & 1.9 & 1.8 & 10.7 & 17.8 & $\ldots$ & $\ldots$ & $\ldots$ & $\ldots$ \\
\hline
\end{tabular}

Sources: Rwandese authorities; and Fund staff estimates and projections.

$1 /$ Proposed.

2/ Net foreign assets as defined in the TMU.

3/ Evaluated at the following program exchange rates: For 2003: RF 511.9/US\$; for 2004: RF 580.3/US\$.

4/ Until December 2003: Three-week moving average around the last Friday of the month. In 2004: end-month number

5/ From June 2002: Includes financial balances of local government.

6/ The domestic fiscal balance is defined as total revenue (excluding privatization proceeds) minus current expenditure (excluding scheduled interest payments.

on external debt) minus domestically financed capital expenditure minus net lending.

7/ According to the TMU. Definition of this aggregate changed in 2002.

8/ Ceiling on contracting or guaranteeing by the central government, local governments, or the NBR of new nonconcessional external debt with original maturity of more than one year. The term debt shall be understood as defined in the Executive Board decision No. 6230-(79/140) adopted August 3, 1979, as amended by Decision No. 11096-(95/100) of October 25, 1995 and Decision No. 12274-(00/85) adopted August 24, 2000. Debt rescheduling and restructuring are excluded from the borrowing limits. Includes financial leases and other instruments giving rise to external liabilities, contingent or otherwise, on nonconcess ional terms. In determining the level of concessionality of these obligations, the definition of concessional borrowing shall apply. Concessional borrowing is defined as having a grant element of 35 percent or more until September 2000 , and 50 percent or more from December 2000 onward. For loans with a maturity of at least 15 years, the ten-year average commercial interest reference rates (CIRRs) published by the OECD should be used as the discount rate for assessing the level of concessionality, while the six-month average CIRRs should be used for loans with shorter maturities. To both the ten-year and the six-month averages, the following margins for differing repayment periods whle the 9/ A US\$5 million OPEC Fund loan to cofinance, jointly with BADEA, the rehabilitation of three hydroelectric projects, with a grant element of less than 50 percent, was reduced to US $\$ 3$ million March 2004. BADEA's Board is scheduled to consider a counterpart US $\$ 2$ million increase that would bring the overall concessionality of financing for the project to 50 percent (evaluated at the Sept. 2002 reference interest rate) by end-June 2004

10/ Ceiling on oustanding stock of external debt (excluding normal import-related credits) owed or guraranteed by the central government, local government, or the NBR with original maturity of up to, and including, one year. Figures in millions of U.S. dollars.

11/ This is a continuous performance criterion, implying that the stock of outstanding nonreschedulable external arrears is expected to be constantly kept at zero throughout the program period. $12 /$ In 2003 and 2004: evaluated at the program exchange rates of RF 511.9/US\$ and RF 580.3/US\$, respectively.

13/ June 2003 datum does not adjust for a temporary credit union deposit (RF 8.8 billion).

14/ Extended broad money is defined as broad money plus depos its in credit unions and credit cooperatives, and rural and agricultural banks (other banking institutions). 
Table 2. Rwanda: Proposal for Structural Conditionality Until the Third Review of the PRGF-Supported Program, 2002-04

\section{Action}

- Ratify a revised 2002 budget in parliament reflecting the understandings reached during the program discussions; including the following elements: $\circ$ import tariff bands at $0,5,15$, and 30 percent in line with initial CET;

$\circ$ VAT rate increased from 15 percent to 17 percent; and

o reduction in the corporate income tax rate from 40 percent to 35 percent, announced.

- Bring reserve money to or below indicative ceiling for end-June 2002.

- Issue guidelines determining qualification and priority for payment of outstanding government obligations for payment, eliminating discretion.

- Enact a budget for 2003, which specifically contains the following elements: 0 excise tax on sales of new and used cars, with rates of 5, 10, and 15 percent, depending on engine size (less than $1500 \mathrm{cc}, 1500 \mathrm{cc}$ to 2500 cc, and above $2500 \mathrm{cc}$ ), on vehicle sales.

o reform the Tax on Professional Remuneration (TPR) Law to make all salary allowances in cash and in kind fully subject to the TPR. Pass the reform in form of an amended TPR Law.

- Revoke the decrease in the beer excise tax rate and start collecting again at a rate of 57 percent, if revenue collection during June-October 2002 does not meet the target set out in the TMU.

- Finalize restructuring plan for a specified commercial bank consistent with understandings with IMF staff.

- Start publishing statistics of government financial operations, following the Government Finance Statistics (GFS) format, on a quarterly basis.

- Incorporate any extrabudgetary and off-budget projects and transactions identified by the recent stocktaking exercise into the budget to the extent appropriate.

- Develop and implement a mechanism to ensure that all borrowing by district governments is reported to the central government on a monthly basis.

- To improve the management of the large volume of nonperforming loans, commission a comprehensive financial sector study, together with the World Bank.
Timing

$7 / 1 / 02$

$6 / 30 / 02$

$7 / 1 / 02$

$1 / 1 / 03$

$9 / 30 / 02$

$10 / 31 / 02$

$12 / 31 / 02$

$12 / 31 / 02$

Tenders to be awarded no later than $7 / 31 / 02$
Prior action $\mathrm{PRGF}^{2}$ (met)

Prior action $\mathrm{PRGF}^{2}$ (met)

Structural performance criterion First review (met)

Structural benchmark First review (met)

Structural benchmark First review (met)

Structural benchmark First review (not met/in progress)

Structural benchmark First review (not met/in progress)

Structural benchmark First review (not met/implemented in November 2002) 
Table 2. Rwanda: Proposal for Structural Conditionality Until the Third Review of the PRGF-Supported Program, 2002-04

- Conduct full audits of three banks.

Action

Timing

- Ensure that the National Bank of Rwanda (NBR), the Ministry of Finance and Economic Planning, the Ministry of Justice, and the Bankers' Association will jointly prepare an action plan to improve the legal environment to facilitate stronger loan recovery.

- Ministry of Finance and Economic Planning to establish standard operating procedures for the conduct of annual audits of the NBR.

- Complete a comprehensive review of all tax exonerations, exemptions and incentives under tax laws and investment agreements; and remove and/or modify such special treatment

- Complete the report on implementation of the 2002 development budget

- Issue the tender for the sale of Rwanda Commercial Bank (BCR)

- Issue list of overdue obligations scheduled for clearance during 2003

- Prepare financial instructions in order to promote effective expenditure control.

- Establish written procedures to ensure that monetary data used for program monitoring purposes are in accordance with the TMU and can be reconciled to the accounting records. External audit firm to complete, subsequent to the completion of the audit of the NBR's 2002 financial statements, a review of the consistency between data reported to the IMF and the audited financial statements.

- Issue action plan for the closure of dormant accounts and accounts operating outside of controlling regulations
$12 / 31 / 02$

12/31/02

$6 / 30 / 03$

Status $^{1}$

Structural benchmark

First review

(met)

Structural benchmark

First review

(not met/implemented in 2003)

$5 / 31 / 03$

Prior action

First review $^{3}$

(met)

$6 / 30 / 03$

Structural performance criterion

Second review

(met)

Structural benchmark Second review (not met/implemented in

September 2003)

$6 / 30 / 03$

Structural benchmark

Second review

(not met, in progress)

$6 / 30 / 03$

Structural benchmark

Second review

(not met/implemented in

September 2003)

7/31/03

Structural performance criterion

Second review

(met)

$8 / 31 / 03$

Structural benchmark

Second review

(not met/in progress)

9/30/03

Structural benchmark Second review (met) 
Table 2. Rwanda: Proposal for Structural Conditionality Until the Third Review of the PRGF-Supported Program, 2002-04

\section{Action}

- Implement a monthly reporting mechanism for the financial operations of all districts

- Submit Organic Budget Law to parliament

- Submit revised investment code to parliament, repealing indirect and direct tax provisions of the code, excepting those of a purely administrative nature, and removing the discretionary authority of the Rwanda Investment Promotion Agency to issue tax incentives.

- Operationalize the NBR's Internal Audit Department by (i) restricting the director's responsibilities to internal audit matters; (ii) NBR Board adopting an audit charter; (iii) recruiting two qualified and experienced internal audit staff members; (iv) completing an audit risk assessment of all NBR operations; (v) auditing program data as of September 30, 2003; and (vi) preparation of a plan for 2004 audit activities approved by the Governor.

- Issue financial instructions in order to promote effective expenditure control.

- Incorporation of tax incentives into the structure of the income tax, applicable, in principle, to all taxpayers.

- Cabinet approval of a new procurement code

- NBR to establish written procedures ensuring data reported to the IMF for program purposes are consistent with the TMU and reconciled with accounting records

- NBR regulation on the net open foreign exchange position of commercial banks to be restored to the text in force in June 2003

- External audit firm to complete the audit of the NBR's 2003 financial statements

- Submit revised 2004 budget to Parliament

- Finalization of action plans, including progressive penalties, for bringing commercial banks into full compliance with banking regulations by $12 / 31 / 04$

- Cabinet approval for export promotion action plan
$9 / 30 / 03$

$12 / 31 / 03$

Timing

Status

Structural benchmark

Second review

(not met/in progress)

Structural benchmark

Second review

(not met/in progress)

$12 / 31 / 03$
Structural performance criterion

Third review (not met/in progress)

Structural benchmark Third review (met)

Structural performance criterion

Third review (not met/in progress)

Structural performance criterion Third review (not met/in progress)

Prior action ${ }^{4,5}$

Prior action ${ }^{4,5}$

Prior action ${ }^{4,5}$

Structural benchmark Fourth review ${ }^{4}$

Structural performance criterion

Fourth review ${ }^{4}$

Structural benchmark Fourth review ${ }^{4}$

Structural benchmark Fourth review ${ }^{4}$ 
Table 2. Rwanda: Proposal for Structural Conditionality Until the Third Review of the PRGF-Supported Program, 2002-04

Action Timing Status

- Monthly reconciliation statements for government financial statements to be published on a quarterly basis, with no more than a one-month lag

- Statements, including tax expenditure, assets and liabilities, public enterprise finances, government equity holdings; consolidated district government budget; and list of government contingent liabilities to be included in the 2005 budget

${ }^{1}$ The disbursements of the second and third loan under the new PRGF arrangement are conditional upon completion of the first and second reviews, respectively.

${ }^{2}$ Prior actions for publication of Executive Board documents for the decision on the August 2002 PRGF arrangement.

${ }^{3}$ Prior actions for publication of Executive Board documents for the completion of the first review.

${ }^{4}$ Newly proposed measure.

${ }^{5}$ Prior actions for publication of Executive Board documents for the completion of the second and third reviews. 


\section{Technical Memorandum of Understanding Between the Government of Rwanda and the International Monetary Fund}

May 20, 2004

1. This memorandum outlines the understandings between the Rwandese authorities and the IMF mission with regard to the definitions of the quantitative and structural performance criteria, and quantitative benchmarks and indicators for the three-year Poverty Reduction and Growth Facility (PRGF) arrangement. It also sets out the modalities and data reporting requirements for monitoring the program. ${ }^{1}$

2. Revisions to the definitions since the last version of the Technical Memorandum of Understanding (TMU) have been made in the following areas: external budgetary support; net foreign assets; net credit to government; domestic fiscal balance; reserve money, and broad money; nonconcessional external debt, short term external debt; and data requirements for the monetary sector and electronic data reporting.

\section{Target Variables Under the Program}

\section{A. External Budgetary Support}

3. Definition: External budgetary support is defined as all official external grants and official external loans to the central government (including all expected or received HIPC Initiative-related grants), except for external grants and loans related to the development budget. In case a program is over financed (negative financing gap), programmed external budgetary support refers only to that level of external budget support needed to close the financing gap to exactly zero at the time of the agreement.

4. Reporting requirement: Data on external budgetary support, separately detailing grant and loan inflows, will be transmitted to the African Department of the IMF on a monthly basis within three weeks of the end of each month.

\section{B. Net Foreign Assets of the National Bank of Rwanda (NBR)}

5. Definition: Net foreign assets of the NBR in Rwanda francs are defined, consistent with the definition of the Special Data Dissemination Standards (SDDS) template, as external assets readily available to, or controlled by, the National Bank of Rwanda (NBR) net of external liabilities of the NBR. Pledged or otherwise encumbered reserves assets including, but not limited to, reserve assets used as collateral or guarantee for third party external liabilities, are to be excluded. Foreign assets and foreign liabilities in U.S. dollars are converted to Rwanda francs by using the U.S. dollar/Rwanda franc program exchange rate. ${ }^{2}$

\footnotetext{
${ }^{1}$ A summary of reporting requirements is provided in Table 1.

${ }^{2}$ The program exchange rate for the 2004 program is set at RF $580.3=\mathrm{US} \$ 1$.
} 
Foreign assets and liabilities in other currencies are converted to U.S. dollars by using the actual end-of-period U.S. dollar/currency exchange rate. Foreign liabilities include, inter alia, use of IMF resources (CCFF and post-conflict emergency assistance purchases and $\mathrm{SAF} / \mathrm{ESAF} / \mathrm{PRGF}$ disbursements).

6. Target and adjustments: The program sets a floor on net foreign assets of the NBR (as a performance criterion or benchmark depending on the test date). In case of higher than programmed inflows of general external budgetary support, excess amounts are targeted to be saved as reserves. The program floor on net foreign assets will thus be increased by any positive difference between actual and programmed general budgetary support. Shortfalls of less than US\$20 million of the programmed level of external budgetary support, as defined in paragraph 3, for end-September 2004, and US\$25 million of the programmed level for external budgetary support for end-December 2004 will not be adjusted. The program floor on net foreign assets will be adjusted downward by the amount of any incremental shortfall in external budgetary support above US\$20 million at end-September 2004, and above US\$25 million at end-December, up to a maximum adjustor of US\$30 million, evaluated at the program exchange rate.

7. Reporting requirement: Data on foreign assets and foreign liabilities of the NBR will be transmitted to the African Department of the IMF on a weekly basis within seven days of the end of each week; Data on the NBR's foreign exchange liabilities to commercial banks (held as required reserves with the NBR) and the exchange rate used for their conversion into Rwanda francs will be shown separately.

\section{Net Credit to Government (NCG)}

8. Definition: Net credit to government from the banking system is defined as the difference between:

(a) credit to government from the banking system, including credit to central government, provinces and districts, outstanding central government debt instruments; government debt to the NBR incurred as a result of the 1995 devaluation (RF 9 billion) and the overdraft to the prewar government (RF 2 billion), and

(b) total government deposits with the banking system, including central government (including the fund for assistance to genocide survivors), provinces and districts, project accounts, counterpart funds, fonds publics affectés, and privatization proceeds with the NBR. The central government comprises treasury and line ministries.

NCG is not affected by credit to or deposits of public enterprises and autonomous public agencies.

9. Reclassifications: The reclassification described in Annex B - for the reclassification of deposits with the NBR of the 15 newly identified autonomous public agencies - affect net credit to the government from the banking system. 
10. Target and adjustments: The program sets a ceiling on NCG (as performance criterion or benchmark) at the test dates. In case of higher than programmed inflows of general external budgetary support, excess amounts are targeted to be saved as government deposits. The program ceiling on NCG will thus be decreased by any positive difference between actual and programmed general budgetary support inflows. The program ceiling on net credit to government will be adjusted upward by the amount of the incremental shortfall in external budgetary support above US\$11 million at end-September 2004, and above US\$24 million at end-December, up to a maximum adjustor of US\$30 million. The NCG adjustor for budgetary support will be evaluated in Rwanda francs at the program exchange rate.

11. Reporting requirement: Data on net credit to central government (showing separately treasury bills and government bonds outstanding, other government debt, and central government deposits) will be transmitted on a monthly basis within three weeks of the end of each month. Deposits of the government with the NBR and with the commercial banks will be separated from the deposits of the public enterprises and autonomous public agencies.

\section{Reserve Money}

12. Definition: Reserve money for the monetary program is defined as currency in circulation, reserves in deposit money banks (excluding National Bank of Rwanda (NBR) borrowing from deposit money banks on the money market but including cash in vault held by commercial banks), deposits of public enterprises (including Caisse Sociale de Rwanda (CSR) and other autonomous public agencies (dépôts des établissements publics assimilés à l'état), deposits of nonbank financial institutions, and deposits of the private sector (autres sommes dues à la clientèle are included in reserve money).

13. Corollary: Borrowing by the NBR from the commercial banks on the money market is included under the net domestic assets of the NBR. More specifically, borrowing by the NBR from the commercial banks on the money market is netted out from commercial bank borrowing from the NBR. However, for balances with respect to deposit money banks, the money market balances of the NBR are excluded from reserve money supply when they are excluded from use in meeting reserve requirements.

14. Definition: The definition of reserve money as performance criterion or benchmark will exclude from the above definition the deposits of the Caisse d'Épargne du Rwanda (C.E.R.) with the NBR, the import deposits placed at the NBR (cautions à l'importation), and the dormant accounts. However, the import deposits are only excluded from this definition up to a maximum amount of FR 150 million, and the maximum amount for the deductible C.E.R. deposits is RF 1 billion.

15. Target and adjustments: The program sets a ceiling on reserve money (as performance criterion or benchmark) at the test dates. If the required reserve ratio of the NBR is lowered, the NBR will be expected to absorb the excess liquidity that this change creates. 
Therefore the reserve money target of the NBR will be adjusted by the absolute change in the ratio times the deposit base of the commercial banks.

16. Reporting requirement: Data on reserve money will be transmitted to the African Department of the IMF on a weekly basis within seven days of the end of each week. This transmission will include a weekly balance sheet of the NBR which will show all items listed above in the definitions of reserve money.

\section{E. Broad Money}

17. Definition: Broad money is defined as the sum of currency in circulation, deposits in commercial banks and nonbank deposits in the NBR. In addition, extended broad money is defined as broad money plus deposits in credit unions and credit cooperatives (UBPR), and rural and agricultural banks (BRD; other banking institutions).

18. Target: There is no performance criterion or benchmark on broad money or extended broad money but given its key influential role on inflation, they will be followed closely as indicative targets.

19. Reporting requirement: The balance sheet of the NBR will be transmitted on a weekly basis within seven days of the end of each week. The balance sheets of the commercial banks and of the other banking institutions, both for the individual institutions and for the respective sector in aggregate, and the monetary survey, will be transmitted monthly within three weeks of the end of each month. The monthly transmission will also include a monthly balance sheet for the NBR which will show all items shown also in the weekly balance sheet for the NBR.

\section{F. Ceiling on Contracting or Guaranteeing by the Central Government, Local Governments, or the NBR of New Nonconcessional External Debt with Original Maturity of More Than One Year}

20. Definition: This performance criterion applies to the contracting or guaranteeing by the central government, local governments, or the NBR of new nonconcessional external debt (as specified below) with original maturity of more than one year, including commitments contracted or guaranteed for which value has not been received. The term debt shall be understood as defined in the Executive Board decision No. 6230-(79/140) adopted August 3, 1979, as amended by Decision No. 11096-(95/100) of October 25, 1995 and Decision No. 12274-(00/85) adopted August 24, 2000. Debt rescheduling and restructuring are excluded from the criterion. Included are financial leases and other instruments giving rise to external liabilities, contingent or otherwise, on nonconcessional terms. In determining the level of concessionality of these obligations, the definition of concessional borrowing shall apply. Concessional debt is defined as having a grant element of 50 percent or more. For loans with a maturity of at least 15 years, the 10-year average commercial interest reference rates (CIRRs) published by the OECD should be used as the discount rate for assessing the level of concessionality, while the 6-month average CIRRs should be used for loans with shorter maturities. To both the 10 -year and the 6-month averages, the following 
margins for differing repayment periods should be added: 0.75 percent for repayment periods of less than 15 years; 1 percent for 15-19 years; 1.15 percent for 20-29 years; and 1.25 percent for 30 years or more. The performance criterion is defined to exclude the use of Fund resources.

21. In addition, loans contracted with the Arab Bank for Economic Development in Africa (BADEA) and the OPEC Fund for energy rehabilitation financing, contracted in 2002 and 2003, and supplemental BADEA lending for this project in 2004 that is intended to improve the overall concessionality of financing for the project, will evaluated using the reference interest rates prevailing in September 2002.

22. Target: The program sets a performance criterion on the ceiling on the contracting or guaranteeing by the central government, local governments, or the NBR of new nonconcessional external debt with original maturity of more than one year.

23. Reporting requirement: Details of all new external debt, including government guarantees, will be provided on a monthly basis within three weeks of the end of each month.

\section{G. Ceiling on Change in Outstanding Stock of External Debt, Owed or Guaranteed by the Central Government, Local Governments, or the NBR with Original Maturity of Up To and Including One Year}

24. Definition: The term "debt" has the meaning set forth in point No. 9 of the Guidelines on Performance Criteria with respect to Foreign Debt adopted on August 24, 2000. Excluded from this performance criterion are normal import-related credits. Normal import-related credits are liabilities that arise from the direct extension, during the normal course of trading, of credit from a supplier to a purchaser - that is, when payment of goods and services is made at a time that differs from the time when ownership of the underlying goods or services changes. Normal import credit arrangements covered by this exclusion will contain pre-specified limits on the amounts involved and the times at which payments must be made. Normal import credits will not involve the issuance of securities. Funding provided by an enterprise other than the supplier for the purpose of purchasing goods or services will not benefit from the exclusion under this performance criterion."

25. Target: The program sets a continuous performance criterion on the ceiling on change in the outstanding stock of external debt, owed or guaranteed by central government, local governments, or the NBR with original maturity of up to and including one year.

26. Reporting requirement: Data on debt and guarantees by central government, local governments, or NBR will be transmitted, with detailed explanations, on a monthly basis within three weeks of the end of each month. 


\section{H. Domestic Fiscal Balance}

27. Definition: The domestic fiscal balance is defined as domestic revenue (excluding grants and privatization proceeds) minus current expenditure (excluding external interest due) and domestically financed capital expenditure on a payment order basis, minus net lending.

28. Target and adjustments: The program sets a ceiling on the domestic fiscal deficit, i.e., a floor on the domestic fiscal balance (as performance criterion or benchmark). As an adjustment, any shortfall in expenditure under the World Bank led demobilization and reintegration program will be used to reduce the deficit target, i.e. will be added to the target for the domestic fiscal balance. The deficit ceiling will be revised downward by the Rwanda franc equivalent of shortfalls in external budgetary grant inflows from the programmed level, evaluated at the program exchange rate, up to a maximum of US\$11 million at endSeptember 2004 and up to a maximum of US\$25 million end-December 2004. In addition, the deficit ceiling will be reduced by the amount of privatization revenue (recorded under net lending).

29. Reporting requirement: Data on domestic revenue, current expenditure, domestically financed capital expenditure and net lending will be transmitted, with detailed explanations, on a monthly basis within four weeks of the end of each month.

\section{Priority Expenditure (Table 2)}

30. Definition: Central government priority expenditure is defined as the sum of those outlays in the recurrent budget and of the community development fund that the government has identified as priority spending in line with the PRSP process. Table 2 provides the list of budget lines under this definition.

31. Target: The program sets a floor on priority expenditure (as performance criterion or benchmark).

32. Reporting requirement: Data on priority expenditure, at the same level of detail as in Table 2 will be transmitted on a monthly basis within three weeks of the end of each month.

\section{J. Net Accumulation of Domestic Arrears}

33. Definitions: Net accumulation of arrears for any given calendar year is defined as the difference between

gross accumulation of new domestic arrears within the calendar year of consideration, cumulative from January 1 to December 31, as measured as the difference between payment orders and actual payments, and 
gross repayment during the calendar year of consideration of any arrears outstanding

at 31 December of the preceding year, including repayment of the preceding year's

float and repayment of older arrears in accordance with the government guidelines.

34. Target and adjustments: The program sets a ceiling on the net accumulation of domestic arrears, with a negative target thus representing a floor on net repayment (as performance criterion or benchmark). The ceiling will be reduced downward by the amount that the excess of gross accumulated bills payable above RF 1 billion at end-June, RF 1.5 billion end-September 2004, and the excess above RF 2 billion at end-December 2004.

35. Reporting requirement: Detailed data on repayment of domestic arrears and the remaining previous-year stock of arrears will be transmitted on a monthly basis within three weeks of the end of each month.

\section{K. Stock of Outstanding Nonreschedulable External Arrears Owed by the Central Government or the NBR}

36. Definition: Nonreschedulable external arrears are defined as the sum of arrears owed by the central government or the NBR to multilateral creditors and, if any, nonreschedulable arrears, to bilateral official and commercial creditors.

37. Target: The program sets a continuous performance criterion on the nonaccumulation of nonreschedulable external arrears.

38. Reporting requirement: Detailed information on repayment and/or refinancing (including the terms of refinancing) of arrears will be transmitted on a quarterly basis within three weeks of the end of each quarter. The Fund will be notified immediately in case of incurrence of any nonreschedulable external arrears.

\section{OTHER DATA REQUirements FOR Program MONITORING}

\section{A. Public Finance}

39. Reporting requirement: Monthly data on external budgetary support with a breakdown of loans by creditor and grants by donor and domestic nonbank financing of the budget (including treasury bills and government bonds held by the nonbank public) will be transmitted on a monthly basis within three weeks of the end of each month; quarterly data on the implementation of the development budget with detailed information on the sources of financing will be transmitted on a quarterly basis within three weeks of the end of each quarter; public sector external and domestic scheduled debt service and payments will be transmitted on a monthly basis within three weeks of the end of each month. The Rwanda Revenue Authority will transmit any updated census results of small and medium enterprises (including the economic characteristics of these enterprises and their estimated annual sales). 


\section{B. Monetary Sector}

40. Reporting requirement: The following data will be transmitted on a monthly basis within three weeks of the end of the month: the individual balance sheets and the consolidated balance sheet of deposit money banks; the individual and consolidated balance sheets of the other bank institutions; the monetary survey (situation monétaire intégrée); disaggregated data on "other items net" of the NBR and deposit money banks; required reserves and excess reserves of individual commercial banks, showing separately foreign exchange held as required reserves with the NBR; development bond and treasury bill holdings of individual commercial banks; nontreasury government deposits at individual commercial banks; nonperforming loans of individual commercial banks; required and actual provisioning of impaired assets for individual banks; capital adequacy ratio for individual commercial banks a weighted average for all commercial banks; and sanctions issued to banks. ${ }^{3}$ Data on the opening and closing balances, and debits and credits of government treasury (OTR) accounts, as well as accounts of the demobilization commission, the Rwanda Revenue Authority (RRA), Fund for Genocide Survivors (FARG), the Road Fund, the Electoral Commission and the Gacaca Commission in the central bank and commercial banks will also be communicated on a quarterly basis.

\section{Public Enterprises}

41. Definition: The financial statements and bank deposits of the key public enterprises (including Rwandatel, Electrogaz, Ocircafé, Ocirthé, and ONP) will be monitored under the program.

42. Reporting requirement: The financial accounts (including profit and loss accounts, balance sheets, and annual reports when published) of key public enterprises (including Rwandatel, Electrogaz, Ocircafé, Ocirthé, and ONP) will be transmitted to the African Department of the Fund within four weeks on a semi-annual basis or as the accounts become available. The statement of these enterprises' bank deposits (bank by bank) will be transmitted to the African Department of the Fund on a quarterly basis within four weeks of the end of each month.

\section{External Sector}

43. Reporting requirement: The following buying, selling, and average exchange rates will be transmitted on a weekly basis within seven days of the end of each week:

(i) intervention exchange rates used in NBR's operations with the commercial banks; (ii) the exchange rates used in interbank transactions among the commercial banks; (iii) the average of (i) and (ii); (iv) the exchange rates for transaction in banknotes at the commercial banks;

\footnotetext{
${ }^{3}$ Detailed data account by account on central government (including ministries), other public agencies, and public enterprises accounts with the NBR and each commercial bank will be transmitted on a quarterly basis within for 4 weeks of the end of the quarter.
} 
(v) the same for foreign exchange bureaus; and (vi) the parallel (black) market exchange rates. All these exchange rates will be calculated on the basis of daily buying and selling rates; the average exchange rates will be calculated on the basis of a simple average of the daily buying and selling rates. The NBR will report weekly on the difference between the parallel market rate (buying and selling) and the weighted weekly average rates of NBR intervention in the interbank market for purchases and sales, respectively.

44. The following data will be provided on a monthly basis within four weeks of the end of each month:

- $\quad$ The amount of foreign exchange held by commercial banks with the NBR as required reserves

- $\quad$ net open foreign exchange position of each commercial bank and foreign exchange bureau, and the calculation method;

- $\quad$ foreign exchange intervention by the NBR on interbank market;

- $\quad$ imports, sales, and purchases of foreign exchange banknotes by commercial banks;

- $\quad$ sales and purchases of foreign exchange banknotes by foreign exchange bureaus.

Export and import data, including volumes and prices, will be transmitted on a monthly basis within four weeks of the end of each month; other balance of payments data including the data on services, official and private transfers, capital account transactions, and the repatriation of export receipts will be transmitted on a quarterly basis within four weeks of the end of each quarter.

\section{E. Real Sector}

45. Reporting requirement: Monthly disaggregated consumer price indices for Kigali (NBR), urban areas (Ministry of Finance), and rural areas (Ministry of Finance) will be transmitted on a monthly basis within four weeks of the end of each month; any revisions to gross domestic product by sector estimates will be transmitted within three weeks of the date of revision. Beginning with data for January 2004, disaggregated consumer price for a new national consumer price index will also be communicated on a monthly basis within four weeks of the end of each month.

\section{F. Electronic Data Reporting}

46. Reporting requirement: The following data will, where feasible, be made available through electronic format (Excel) and e-mailed to the African Department of the Fund:

(i) Monetary data and exchange rates: 
Monthly balance sheet of the NBR, summary balance sheet of the commercial banks, individual balance sheets of the commercial banks, details of public sector deposits with commercial banks, details of commercial banks' loan provisioning and capital adequacy, monthly data on foreign exchange operations of commercial banks and the NBR, and net open foreign exchange positions. These data will be transmitted within three weeks of the end of the month.

Quarterly reporting on opening and closing balances as well as debits and credits of OTR accounts of, the demobilization commission, RRA, FARG, the Road Fund, the Electoral Commission and Gacaca Commission in the central bank and commercial banks.

Weekly balance sheet of the NBR will be transmitted within seven days of the end of each week.

Weekly data on NBR interventions on the money market (appel d'offres) both to inject and to absorb liquidity, including the maturity and the due date of the transactions, the amounts offered, demanded, and allocated (by bank, in millions of Rwanda francs), the maximum, minimum, marginal, and average interest rates offered, and the interest payments (by bank, in Rwanda francs). These data will be made available within seven days after the end of the week.

Weekly data on recourse to the discount window (prise en pension), including the period of borrowing, the discount rate, and the amount (by bank, in Rwanda francs). These data will be made available within seven days after the end of the week.

Weekly update of the monthly treasury plan (plan de trésorérie) for foreign exchange reserves at the NBR. These data will be made available within seven days after the end of the week.

Weekly data on exchange rates, including foreign exchange auctions by the NBR, the amount of foreign exchange offered, demanded, and allocated (by commercial bank, in U.S. dollars and Rwanda francs), and the minimum, maximum, marginal, and average exchange rate offered. These data will be made available within seven days after the end of the week.

Daily balance by commercial bank of amounts outstanding from money market interventions to absorb liquidity (appel d'offres-ponction), to inject liquidity (appel d'offres-injection), under the discount window (prise en pension) and any other credit facility of the NBR, respectively. These data will be made available within seven days of the reported date.

Weekly balance of the subaccount for HIPC Initiative assistance from the IMF at the NBR. The data will be provided within seven days of the end of the week. 
(ii) Fiscal "flash" report, including detailed lists of priority and exceptional expenditure. These data will be transmitted within four weeks of the end of the month.

(iii) Detailed export and import data; and

(iv) Detailed CPI data.

\section{Program Monitoring Committee}

47. Definition: The Interministerial Technical Committee, composed of senior officials of key ministries and the National Bank of Rwanda shall meet once a month and be responsible for monitoring the performance under the program, informing the IMF staff regularly about progress on program implementation, and transmitting supporting information necessary for program monitoring.

48. Reporting requirement: The names of the Interministerial Technical Committee shall be communicated to the IMF no later than the date of submission of the authorities' request for support of the three-year PRGF-supported program to the Executive Board of the IMF or the start of a new annual arrangement. The Interministerial Technical Committee shall provide to the IMF staff a progress report on the program implementation on a monthly basis within four weeks of the end of each month.

\section{AnNex B. ReClassifications}

The following reclassification of data has been made to the monetary survey:

Reclassification of the deposits of $\mathbf{1 5}$ additional autonomous public agencies: In tables presented by the IMF prior to November 5, 2000, deposits of the central government with the NBR included deposits of 15 autonomous agencies. As of November 6, 2000 these deposits will be itemized separately in a category called "public nongovernment deposits," but will still be included in the domestic credit of the NBR. 
Table 1. Rwanda: Summary of Reporting Requirements

\begin{tabular}{|c|c|c|c|c|}
\hline Status & Variable or Table & $\begin{array}{l}\text { Reporting } \\
\text { Frequency }\end{array}$ & $\begin{array}{l}\text { Reporting } \\
\text { Delay from } \\
\text { End of Period } \\
\text { Covered }\end{array}$ & $\begin{array}{l}\text { Report Data } \\
\text { Electronically }\end{array}$ \\
\hline & A. Monetary and Foreign Exchange & & & \\
\hline $\mathrm{PC}$ & $\begin{array}{l}\text { Net foreign assets National Bank of Rwanda } \\
\text { (NBR) }\end{array}$ & Weekly & Seven days & Yes \\
\hline $\mathrm{PC}$ & Reserve money & Weekly & Seven days & Yes \\
\hline $\mathrm{PC}$ & Net credit to central government & Monthly & Three weeks & Yes \\
\hline Table & Monthly balance sheet of the NBR & Monthly & Three weeks & Yes \\
\hline Table & Summary balance sheet of the commercial banks & Monthly & Three weeks & Yes \\
\hline Table & Individual balance sheets of the commercial banks & Monthly & Three weeks & Yes \\
\hline Table & $\begin{array}{l}\text { Details of public sector deposits with individual } \\
\text { commercial banks }\end{array}$ & Quarterly & Three weeks & Yes \\
\hline Table & $\begin{array}{l}\text { Opening and closing balances as well as debits } \\
\text { and credits for OTR accounts, the demobilization } \\
\text { commission, Rwanda Revenue Authority (RRA), } \\
\text { Victims of Genocide Fund (FARG), the Road } \\
\text { Fund, and Gacaca in the central bank and } \\
\text { commercial banks; }\end{array}$ & Quarterly & Three weeks & Yes \\
\hline Table & $\begin{array}{l}\text { Details of commercial banks' loan provisioning } \\
\text { and capital adequacy }\end{array}$ & Monthly & Three weeks & Yes \\
\hline Table & $\begin{array}{l}\text { Monthly data on foreign exchange operations of } \\
\text { commercial banks, the NBR, and foreign } \\
\text { exchange bureaus }\end{array}$ & Monthly & Three weeks & Yes \\
\hline Table & $\begin{array}{l}\text { Net open foreign exchange positions of } \\
\text { commercial banks and foreign exchange bureaus }\end{array}$ & Monthly & Three weeks & Yes \\
\hline \multirow[t]{2}{*}{ Table } & Exchange rates & Weekly & Seven days & Yes \\
\hline & B. Debt & & & \\
\hline $\mathrm{PC}$ & New external government borrowing & Monthly & Three weeks & \\
\hline $\mathrm{PC}$ & Stock of short-term external government debt & Monthly & Three weeks & \\
\hline & C. Fiscal & & & \\
\hline $\mathrm{PC}$ & $\begin{array}{l}\text { Domestic arrears (repayment of the end-of-year } \\
\text { stock of arrears and accumulation of new arrears) }\end{array}$ & Monthly & Three weeks & Yes \\
\hline $\mathrm{PC}$ & External arrears & $\ldots{ }^{1}$ & $\ldots$ & Yes \\
\hline $\mathrm{OV}$ & $\begin{array}{l}\text { External budgetary support with a break down } \\
\text { between of loans by creditor and grants by donors. }\end{array}$ & Monthly & Three weeks & Yes \\
\hline Table & $\begin{array}{l}\text { Fiscal data (revenue, expenditure, }{ }^{2} \text { priority } \\
\text { expenditure, exceptional expenditure, wage bill) }\end{array}$ & Monthly & Three weeks & Yes \\
\hline Table & Development budget implementation & Quarterly & Three weeks & Yes \\
\hline Table & Scheduled debt service and payments & Quarterly & Four weeks & Yes \\
\hline
\end{tabular}


Table 1. Rwanda: Summary of Reporting Requirements

\begin{tabular}{|c|c|c|c|c|}
\hline Status & Variable or Table & $\begin{array}{l}\text { Reporting } \\
\text { Frequency }\end{array}$ & $\begin{array}{l}\text { Reporting } \\
\text { Delay from } \\
\text { End of Period } \\
\text { Covered }\end{array}$ & $\begin{array}{l}\text { Report Data } \\
\text { Electronically }\end{array}$ \\
\hline Table & Public enterprises financial statements & Semiannual & Four weeks & \\
\hline Table & Public enterprises bank deposits & Quarterly & Four weeks & \\
\hline Table & $\begin{array}{l}\text { Estimated and actual tax payments of the public } \\
\text { enterprises }\end{array}$ & Quarterly & Four weeks & \\
\hline & E. Civil service & & & \\
\hline $\mathrm{OV}$ & $\begin{array}{l}\text { Size of the civil service (core civil service and } \\
\text { teachers) }\end{array}$ & Monthly & Three weeks & Yes \\
\hline & F. Balance of payments & & & \\
\hline Table & Export and imports & Monthly & Four weeks & Yes \\
\hline Table & Detailed Balance of Payments & Quarterly & Four weeks & \\
\hline & G. Prices & & & \\
\hline $\mathrm{OV}$ & CPI Kigali (NBR), urban, and rural (Minecofin) & Monthly & Four weeks & Yes \\
\hline \multicolumn{5}{|c|}{$\begin{array}{l}\text { 1/ The authorities will notify immediately the Fund in case of incurrence of any nonreschedulable external arrears. } \\
2 / \text { On commitment basis (engagement) and on payment order basis (ordonnancement); the provision of fiscal data is } \\
\text { based on the Aflash } \cong \text { reporting (aggregate and by ministry). }\end{array}$} \\
\hline $\begin{array}{l}\mathrm{PC}=\mathrm{p} \\
\mathrm{QI}=\mathrm{q} \\
\mathrm{OV}=\mathrm{c}\end{array}$ & $\begin{array}{l}\text { rmance criterion or quantitative benchmark. } \\
\text { itative indicator. } \\
\text { variable. }\end{array}$ & & & \\
\hline
\end{tabular}


Table 2. Rwanda: Recurrent and Capital Priority Expenditure, 2003 1/ (In millions of Rwanda francs)

\begin{tabular}{|c|c|}
\hline & 2003 \\
\hline Internal affairs & 4,739 \\
\hline National police services & 4,103 \\
\hline Prisons & 636 \\
\hline Agriculture & 2,142 \\
\hline Agricultural production & 1,000 \\
\hline Livestock production & 686 \\
\hline Forestry resources & 192 \\
\hline Soil conservation and water systems management & 92 \\
\hline Agricultural extension and marketing & 172 \\
\hline Commerce & 768 \\
\hline Promotion of trade and commerce & 34 \\
\hline Industrial development and artisanal promotion & 522 \\
\hline Export promotion & 212 \\
\hline Education & 12,879 \\
\hline Pre-primary and primary education & 1,849 \\
\hline Secondary education & 853 \\
\hline Tertiary education & 8,019 \\
\hline Scientific and technological research & 605 \\
\hline Institutional support & 1,552 \\
\hline Youth and Sports & 420 \\
\hline Youth mobilization & 53 \\
\hline Cultural promotion & 172 \\
\hline Research, acquisition, and conservation of the national heritage & 196 \\
\hline Health & 4,601 \\
\hline Primary health care & 1,431 \\
\hline Specialist care for major health problems & 2,359 \\
\hline Development of health structures & 392 \\
\hline Improvement in health management services & 419 \\
\hline Transport and communication, energy and water resources & 3,313 \\
\hline Development and modernization of communication infrastructures & 340 \\
\hline Improvement in transport services & 208 \\
\hline Rationalization and management of urban land & 113 \\
\hline Development of transport infrastructure $2 /$ & 2,275 \\
\hline Energy & 45 \\
\hline Water and sanitation & 150 \\
\hline Mining and other geological programs & 80 \\
\hline Methane gas unit & 103 \\
\hline Gender & 286 \\
\hline Support programs for promotion and development of women & 178 \\
\hline Promotion of gender in development & 52 \\
\hline Promotion of socioeconomic equity & 57 \\
\hline Public service & 501 \\
\hline Civil service reform & 374 \\
\hline Employment and social security promotion & 127 \\
\hline Lands and resettlement & 684 \\
\hline Land planning and management & 377 \\
\hline Planning and supervision of housing amenities & 193 \\
\hline Conservation and protection of the environment & 113 \\
\hline Local government (excluding exceptional expenditure) & 6,448 \\
\hline Decentralization & 249 \\
\hline Community development & 565 \\
\hline Social reinsertion & 49 \\
\hline Family rehabilitation & 14 \\
\hline Mass education & 27 \\
\hline Promotion of children's rights & 18 \\
\hline Decentralization (district transfers in recurrent budget) & 1,526 \\
\hline Common Development Fund (district transfers in development budget) 3/ & 4,000 \\
\hline Provinces & 23,416 \\
\hline Total recurrent & 56,196 \\
\hline Total & 60,196 \\
\hline
\end{tabular}

Source: Rwandese authorities.

1/ All programs are classified as recurrent expenditures, except where marked. 2/ Includes Road Fund.

3/ As part of capital expenditure.

\section{CInternational Monetary Fund. Not for Redistribution}


Table 3. Rwanda: Composition of Exceptional Expenditure, 2003 (In millions of Rwanda francs)

Demobilization/Reintegration/Reinsertion

Supplies for prisoners

Gacaca 1/

Gacaca Sensibilization, Ministry of Justice

Health insurance Gacaca members

Gacaca jurisdictions

Victims of Genocide Fund (FARG)

Orphans assistance

Assistance to vulnerable groups

Reinsertion of vulnerable groups

Support to local initiatives (education)

Support to orphanages and ENA

Reinsertion of displaced groups from Gishwati 180

Reinsertion of street children

CFJM operation

Good governance commissions

Human Rights National Commission

Constitutional Commission

Commission for Unity and Reconciliation

Electoral Commission/Referendum/Elections

Office of the Ombudsman

National Commission for the Fight Against AIDS

Educational institutes

KIST (Kigali Institute for Science and Technology)

KHI (Kigali Health Institute)

KIE (Kigali Institute of Education)

Special exceptional road works

Special exceptional health expenditure

Total

41,797

Source: Rwandese authorities.

1/ Gacaca $=$ community justice initiative 


\section{Rwanda: Relations With the Fund}

As of March 31, 2004

I. Membership Status: Joined: 09/30/1963; Article VIII

II. General Resources Account:

$\underline{\text { SDR million }}$

Quota

80.10

percent Quota

Fund holdings of currency

80.11

100.00

Reserve position in Fund

0.00

100.02

0.00

III. SDR Department:

SDR million

Net cumulative allocation

13.70

percent Allocation

Holdings

20.06

100.00

146.47

IV. Outstanding Purchases and Loans: $\underline{\text { SDR million }}$

PRGF arrangements

61.84

percent Quota

77.20

V. Financial Arrangements:

\begin{tabular}{|c|c|c|c|c|}
\hline Type & $\begin{array}{l}\text { Approval } \\
\underline{\text { date }}\end{array}$ & $\begin{array}{l}\text { Expiration } \\
\underline{\text { date }}\end{array}$ & $\begin{array}{l}\text { Amount approved } \\
\text { (SDR million) }\end{array}$ & $\begin{array}{l}\text { Amount drawn } \\
\text { (SDR million) }\end{array}$ \\
\hline$\overline{\mathrm{PRGF}}$ & $08 / 12 / 2002$ & $08 / 11 / 2005$ & 4.00 & 1.15 \\
\hline ESAF/PRGF & 06/24/1998 & $04 / 30 / 2002$ & 71.40 & 61.88 \\
\hline SAF & 04/24/1991 & $04 / 23 / 1994$ & 30.66 & 8.76 \\
\hline Stand-by & 10/31/1979 & $10 / 30 / 1980$ & 5.00 & 0.00 \\
\hline
\end{tabular}

VI. Projected Obligations to Fund (SDR million; based on existing use of resources and present holdings of SDRs):

\begin{tabular}{|c|c|c|c|c|c|}
\hline & \multicolumn{5}{|c|}{ Forthcoming } \\
\hline & 2004 & 2005 & 2006 & 2007 & 2008 \\
\hline Principal & 3.57 & 6.66 & 10.47 & 12.38 & 11.36 \\
\hline Charges/Interest & 0.30 & 0.28 & 0.24 & 0.18 & 0.12 \\
\hline Total & 3.87 & 6.94 & 10.71 & 12.56 & 11.48 \\
\hline
\end{tabular}


VII. Implementation of HIPC Initiative:

Commitment of HIPC assistance

Decision point date

Assistance committed (end-1999 NPV terms) ${ }^{13}$

Total assistance (US\$million)

Of which: Fund assistance (US\$million)

(SDR equivalent in millions)

Completion point date
Enhanced

framework

$12 / 22 / 2000$

452.00

43.80

33.81

Floating

Delivery of Fund assistance (SDR million)

Amount disbursed

Interim assistance

Completion point

Additional disbursement of interest income ${ }^{14}$

Amount applied against member's obligations (cumulative)

\section{Safeguards Assessments:}

Under the Fund's Safeguards Assessment policy, the Banque Nationale du Rwanda (BNR) is subject to a safeguards assessment under the PRGF arrangement. A Safeguards Assessment was completed on April 14, 2003, and the proposed recommendations have largely been implemented.

\section{Exchange System:}

On March 6, 1995, Rwanda adopted a market-determined exchange rate system. Before then, the Rwanda franc was pegged to the SDR. On December 1998, Rwanda accepted the obligations under Article VIII, Sections 2, 3 and 4, requiring it to ensure that the exchange system is free of restrictions on the making of payments and transfers for current international transactions. In 2001, a foreign exchange auction system was put in place with technical assistance from MAE. Since February 7, 2001, auctions have been taking place on a weekly basis.

${ }^{13} \mathrm{NPV}$ terms at the decision point under the enhanced framework.

${ }^{14}$ Under the enhanced framework, an additional disbursement is made at the completion point corresponding to interest income earned on the amount committed at the decision point but not disbursed during the interim period. 


\section{Article IV Consultation:}

Rwanda is on the revised 24-month consultation cycle. The Executive Board discussed the staff report for the 2002 Article IV Consultation on July 24, 2002.

\section{FSAP Participation, ROSCs, and OFC Assessments:}

A Report on Observance of Standards and Codes on Fiscal Transparency (ROSC) was issued in July 2003. A Financial Sector Assessment Program (FSAP) is planned in the latter half of 2004. Rwanda has not had an Official Financial Center (OFC) assessment.

\section{Technical Assistance:}

1999 FAD long-term experts, on tax policy, on budget preparation, and on treasury management

1999 MAE long-term general advisor to governor of NBR

1999 MAE experts on banking supervision and foreign exchange market operations

2000 FAD experts on budget execution and on tax policy

2000 MAE experts on foreign exchange market operations, and banking supervision

2000 STA mission on money and banking statistics.

2000 STA mission on balance of payments statistics

2001 FAD experts on expenditure management and on tax policy

2001 FAD mission on tax policy

2001 MAE mission on foreign exchange policy, monetary policy, and banking supervision

2001 MAE expert on monetary policy implementation

FAD mission on assessment of tracking of poverty reducing expenditure, and the fiscal ROSC

MAE expert on banking supervision

FAD experts on expenditure management, and on tax policy (until mid-year)

2002 MAE expert on banking supervision (until November)

2002 MAE expert on monetary policy implementation

2002 AFRITAC East work plan mission

2003 AFRITAC East mission on statistical issues

2003 FAD mission on fiscal ROSC and budget management system

2003 AFRITAC East mission on developing the market for government treasury bills

2003 FAD mission on reform of investment incentives and tax reform

2003 STA multi-sector statistics mission on reform of investment incentives and tax reform

2003 FAD mission on Decentralization

2004 FAD tax administration expert on strengthening of revenue administration.

\section{Resident Representatives:}

Mr. Abdikarim Farah has been in Kigali as Resident Representative since January 2001. 


\section{Rwanda: IMF-Bank Relations Annex}

(As of March 31, 2004)

\section{Partnership for Rwanda's Development Strategy}

Donor agencies have been key players in Rwanda since the genocide. With the support from the international community, Rwanda has made notable progress along an ambitious path of reconstruction, national reconciliation and economic reform. In recent years, the Government has made some ambitious efforts, based on its Poverty Reduction Strategy Paper (PRSP), to reduce poverty and improve living conditions of the poor. The PRSP was completed in June 2002. The accompanying PRSP progress report was issued in July 2003. This strategy was supported and discussed by the Boards of the IDA and the IMF on August 12, 2002. The PRSP targeted the halving of poverty by 2015 through a private sector and rural sector strategy. The strategy mainly focuses on six priority areas: (1) rural development and agricultural transformation; (2) human development; (3) economic infrastructure; (4) good governance; (5) private sector development; and (6) institutional capacity building-as the focus for public actions on poverty reduction. Civil society, government agencies and ministries, and donors have all been actively involved in the PRSP process and monitoring.

\section{World Bank Group Program and Portfolio}

The last Country Assistance Strategy for Rwanda was discussed by the World Bank Board in December 2002. The CAS sets out an assistance program consistent with the country's PRSP and emphasizes the need to move progressively from project based approaches to budget support. In line with this approach, a Poverty Reduction Strategy Credit (PRSC) is currently under preparation. The proposed credit would help strengthen GoR capacity to: (i) plan and budget results oriented public sector actions supporting the implementation of the Poverty Reduction Strategy; (ii) develop incentive frameworks through performance-based payments and contracting. (iii) establish strong accountability mechanisms enhancing the capacity of Rwandan citizens to monitor and provide feedback to service providers-both public and private; and (iv) implement a sound fiduciary framework, as well as a monitoring and evaluation system to facilitate transparency and accountability in the service delivery in the sectors of focus (i.e., health, education, water, energy). The credit is expected to go to the Board in September 2004.

International Development Agency (IDA) Program: Since 1970, Rwanda has received 56 IDA credits and grants totaling US\$1,198 million. Disbursements to date from IDA to Rwanda have totaled about US\$907.2 million, with a total undisbursed balance of US\$227 million. Rwanda's current portfolio of eight operations represents, as of March 2004, a total commitment value of US\$261.3 million of credits and one grant facility of US\$30.5 million for an HIV/AIDS project.

Overall, IDA has financed projects in (i) infrastructure, particularly road construction and maintenance, electricity and water supply and sanitation infrastructures; (ii) agriculture, rural development, and forestry; (iii) social infrastructure, including health and population, and education and training; (iv) private sector development, public enterprise reform, financial 
development, and technical assistance; and (v) two policy-based quick-disbursing operations. During the immediate post-genocide period, IDA financed two emergency budget support operations and a social fund-type project, and restructured its prewar portfolio of investment projects to meet the high-priority needs associated with the emergency and the transition from conflict to development.

International Finance Corporation (IFC) Program: The IFC has made some investments in Rwanda, in various industries. Investments have been made in the Rwandan match factory (SORWAL). In FY98, IFC approved a US\$0.53 million investment in Highland Flowers and in FY00, a US\$6.0 million in RWANDACELL. In December 2000, an investment of US\$0.8 million was approved for an apartment hotel in Kigali. In addition to these investments, IFC has also provided some technical assistance support to Rwanda focusing on privatization, SMEs, the financial and industrial sectors.

Multilateral Investment Guarantee Agency (MIGA) Program: Rwanda signed and ratified the MIGA Convention on October 27, 1989. On September 27, 2002, it became a full member of MIGA with the completion of its membership requirements, including payment of the usable currency and the local currency portions of its initial subscription, and deposit of the promissory note. The membership was followed by Rwanda's election to MIGA's Board of Directors during the World Bank/IMF annual meetings held in Washington. As one of its cooperative initiatives, MIGA seconded a senior underwriter for more than a year to COMESA (of which Rwanda is a member) to work on the creation of the Africa Trade Insurance Agency, which was officially launched in Uganda in July 2001. Rwanda is a founding member of ATI, along with seven other COMESA countries. Headquartered in Nairobi, Kenya, ATI provides political risk insurance for trade credits in African countries. All African countries are eligible to participate.

\section{Bank-IMF Collaboration in Specific Areas}

The Bank and Fund are closely coordinating their lending and policy advice to the Government of Rwanda. This collaboration has deepened in recent years especially in the context of the PRSP and HIPC processes.

Areas in which the Fund leads: The IMF leads the policy dialogue on macroeconomic policies, including fiscal and monetary policies. The IMF has supported Rwanda through several arrangements under ESAF/PRGF. The Government had requested a new three-year arrangement, which started in August 2002. The second and third review of the arrangement would go to the IMF Board in May/June, 2004. This new arrangement under the PRGF is to support a comprehensive framework for macroeconomic, structural, and social policies to foster growth and reduce poverty in Rwanda. Structural reforms under the PRGF center around measures to raise revenue to make sufficient room for increased poverty-related spending and development needs, and to strengthen public expenditure management and financial governance.

Areas in which the Bank leads: The Bank leads the policy dialogue on structural and institutional reforms in a number of areas. These include: infrastructure, including roads, communication, water, sanitation, and energy; private sector development; agriculture and 
rural development; social sectors, including health and HIV/AIDS, education, community development, and demobilization and reintegration.

- Broad Structural and Policy reforms: The first structural adjustment loan for Rwanda was approved in June 1991. In 1995 and 1997, the Bank approved two emergency recovery credits (US\$50 million each) as quick-disbursing budget support to assist in the rehabilitation and recovery efforts. In March 1999, an economic recovery credit (ERC) equivalent to US\$75 million was approved to support the economic reform efforts and the overall transition from conflict to peace. The credit was disbursed in three tranches; the last one was at end-March 2001. The Board also approved a supplement to the ERC in the equivalent of US\$15 million in December 2000, which was disbursed in full in March 2001.

Supported by the new CAS, an Institutional Reform Credit (IRC) of US\$85 million was approved on December 2, 2002, with the first tranche of US\$45 million disbursed in December 2002 to support the implementation of the Government's poverty reduction strategy. Since then, many of the key institutional and structural reforms have been identified as triggers for the remaining two tranches. Important measures identified are: adoption of an MTEF approach to budget planning and execution, completion of a Financial Accountability Review and Action Plan, which is now under implementation, privatization of key government undertakings in energy, telecommunications, tea factories, legal reforms to promote gender equality, and privatization of the Banque Commercial de Rwanda (BCR). Although progress on some of these measures have been slow, a number of measures are either at advanced stages or have been completed. Privatization of the BCR - which is a specific condition for the floating tranche - is expected to be completed by June 2004 .

In addition to strengthening the reforms initiated under previous adjustment credits, the upcoming PRSC will focus on the following key areas: (i) pro-poor public expenditures - in education, health, water/sanitation, and energy; (ii) improved quality, efficiency and equity of service delivery; and (iii) policies to promote sustainable economic growth that support increased productivity of rural populations, through increased access to social services, and support to export agriculture.

- Infrastructure and private sector development: A number of Bank-supported infrastructure projects that closed recently helped to strengthen capacity and rebuild infrastructure in post-genocide Rwanda. The Transport Sector Project helped to reform sector policies, develop the planning capacity of the government, improve road maintenance ability, and promote private sector activity in the sector. The project supported main road construction, improved road maintenance, and promotion of communal activities. The Second Communications Project, which closed on June 30, 1999, promoted efficient communications through institutional reform and investments. It sought to create autonomous, commercially oriented operating entities for the telecommunications and postal services, and to provide a regulatory framework to permit the entry of the private sector into the telecommunications industry. The Energy Sector Project helped to establish national energy policies as the 
basis for efficient utilization of Rwanda's energy resources and provided a framework for private sector participation in the sector. The Second Water Supply Project helped to repair damaged water supply systems, financed an evaluation of the water needs in secondary cities, and rehabilitated rural water supply systems. The Rural Water Supply and Sanitation, approved by the Board in June 2000, will continue the activities of the Second Water Supply Project in a framework that emphasizes greater community participation. The Bank is preparing new projects in transport, energy, and urban infrastructure to continue the support of the closed projects. In addition, an upcoming Poverty Reduction Support Credit will support key reforms and policies in water/sanitation, and energy to improve the quality, efficiency and equity of service delivery.

- Private Sector Development: The emphasis of the Bank's support in private sector development has been on enabling the establishment of a liberal institutional, legal, and policy environment to accelerate private sector activity and enhance external competitiveness. In this vein, the Private Sector Development Project, which closed in September 2000, sought to promote expansion of the private sector through the provision of both incentives and means to engage in labor-intensive, export-oriented activities within an improved economic and legal framework. A follow-on project, the Competitiveness and Enterprise Development Project (CEDP), was approved by the Board on April 19, 2001. The CEDP will continue to support the reform of the telecommunications sector and the privatization of the current telephone monopoly, and tea factories. It will also provide support to the energy and water sector by providing management and rehabilitation funds (including management contract fees), and assist the government in privatizing the remaining state-owned enterprises. One of the main components of this project is support to financial sector reforms, which aims at (i) addressing certain issues in the commercial banking sector, and (ii) strengthening the micro-finance sector. Another key component of the project is the support to private sector development of enterprises, associations and institutions that support private sector development including investment promotion and improvement of the business environment in Rwanda. Many of the components are well advanced . In addition, the Regional Trade Facilitation Project (RTFP) aims to contribute to poverty alleviation through private sector led growth in participating countries by improving access to financing for productive transactions and crossborder trade.

- Agriculture and Rural Development: The Bank has been supporting Government efforts through the Rural Sector Support Project, which was approved in March 2001. The aims of this operation is to reduce the smallholders' incentives for autarchy, to promote the adoption of improved inputs, and to raise productivity and incomes among rural populations. Furthermore, the RSSP has been supporting programs that increase agricultural productivity, develop rural agricultural and nonagricultural enterprises, and promote farmers' organizations as agents for the adoption and dissemination of new farming technologies. 
- Social sector: The Bank's activities have been aimed at restoring social infrastructure and services in the aftermath of the 1994 genocide through the Education Sector Project, approved in 1991, and the Health and Population Project, also approved in 1991. The Education Project was finalized in June 2000, and on June 6, 2000 the Board approved a US\$35 million Human Resources Development Project (HRDP) aimed at improving quality and access to education. The Health and Population Project supported the operation of six health districts, the supply of essential drugs, a national information, education, and communications (IEC) capacity, and the restructuring and decentralization of the National AIDS Control Program.

US\$7 million supplemental to this project was approved by the Board at end-2000 to continue activities pending the design of a new project. The Community Reintegration and Development Project (CRDP), approved in October 1998, has promoted community reintegration and development through community participation. CRDP is strengthening the capacity of local communities and the administration at the communal and national levels to identify, design, and implement development projects. This has supported the decentralization process. The Bank recently approved a grant of US\$30.5 million for HIV/AIDS Prevention to support the fight against the pandemic. In addition, the HRDP, and the CEDP have HIV/AIDS components that are used to assist the relevant ministries to spread the message of prevention among their staff and clients. The Demobilization and Reintegration project, which was approved by the Board on April 25, 2002, is to help demobilize and subsequently reintegrate ex-combatants into civilian life. Additional support to reforms in the social sectors, and implementation of the PRSP will be provided through an upcoming PRSC.

- Community Development. The Decentralization and Community Development Project (DCDP) will consolidate a framework in four entire provinces and two districts in a fifth province, to boost the emergence of a dynamic local economy, through communities who are empowered to lead their own development process under an effective local government. This is a key plank in the national poverty reduction strategy and is underpinned by the Government's decentralization policy. The project therefore contributes to Rwanda's long term goal for decentralization and a realigned mandate for central government, through the empowerment and improved accountability of local governments and communities. The project will help to strengthen District capacity for planning, and consultation with local communities. It will also support local procedures for project finance and management, while promoting transparency and public awareness.

Areas of Shared Responsibility: The IMF and the World Bank staff continue to collaborate in supporting the Government's structural reforms in the areas of budgeting, expenditure and financial management, revenue control, and financial sector management.

- Budgeting, public expenditure management and revenue control. A joint BankFund assessment of the Government's capacity to track poverty expenditures was completed in 2001, which highlighted the key areas for improvement. An update of the 2001 exercise is planned for May 2004. IMF technical assistance missions have 
been fielded in the areas of financial management, fiscal decentralization, and revenue management. The Bank has provided support in this area under the Institutional Reform Credit, with the completion of a Financial Accountability Review. Based on the results of this review, a detailed action plan for strengthening the public financial management system was recently completed. This action plan was developed through a highly consultative and participative process that engaged all stakeholders. The Bank will support implementation of the action plan through the IDA Public Sector Capacity Building Project (PSCBP) currently under preparation.

- Public Sector Reform and Capacity Building. The Fund follows public service reforms through their impact on macroeconomic aggregates, and engages in discussion with government on the trade-offs. Currently, the government is in the process of consulting various partners to launch a number of reforms in the sector. DfID as the lead donor in public service reform is preparing a sizable program to support implementation of Rwanda's Public Sector Reform Strategy. The Bank will join DfID and other development partners in supporting public service reform in Rwanda through the Public Sector Capacity Building Project (PSCBP). The PSCBP aims to support public sector entities at the central level to strengthen the capacity for more efficient, effective, transparent and accountable performance of their redefined roles and functions and to achieve their strategic objectives contributing to the PRSP. More specifically, the PSCBP aims to: (i) attract, manage and retain qualified staff, (ii) seek to improve the quality and efficiency of service delivery through support to "contracting for performance" based on functional reviews of all ministries, departments and agencies to identify functions to be contracted out to the private sector and civil society and those to be decentralized and (iii) use financial resources in an effective, transparent and accountable manner towards achieving the objectives of their intended use. PSCBP attention to issues of public accountability and monitoring impacts will further be complemented by the Bank's PRSC.

- Financial Sector. In this area the Bank has provided support in the restructuring of the banking sector, and in addressing the problem of non-performing loans. The Bank has also supported the government to complete reviews of the micro-finance sector, to feed into the development of a sector strategy. Upcoming technical assistance will support the central bank in dealing with issues pertaining to money laundering. The IMF has supported technical assistance in banking supervision and prudential regulation. A Financial Sector Assessment Program is planned later in 2004. Currently, the IMF is providing long-term technical assistance to the Central Bank in monetary policy analysis, banking supervision, and internal audit. The Fund is also providing technical assistance on the development of securities markets.

\section{World Bank staff}

Questions may be referred to Emmanuel Mbi (Tel. 202-473-4953) and Kene Ezemenari (Tel. 202-458-5559). 
Status of World Bank Group Operations in Rwanda

(As of March 31, 2004)

\section{A. Statement of IDA Credits}

$\begin{array}{lll}\text { Status of } & \text { Fiscal } & \text { Purpose } \\ \text { Credit } & \text { Year } & \end{array}$

Closed credits:

(47 credits closed)

Active credits:

Original Credit

Amount in

2000

Rural Water Supply and Sanitation

20.0

US\$Million

(Less

Cancellations)

$794.9^{15}$

2001

Regional Trade Facility

7.5

35.0

48.0

Undisbursed

2000

Human Resource Development

25.0

30.5

34.9

19.2

6.0

2001

Rural Sector Support Project

85.0

24.4

47.2

18.6

2003

HIV/AIDS

2003

Institutional Reform Credit

291.8

225.8

Total: active projects:

$1,228.6$

78.5

Of which: repaid

872.9

45.0

Total held by the Bank and IDA

\section{B. Statement of IFC Investments in Rwanda}

The IFC's pending commitments in Rwanda comprise those in Rwandacell, AEF Dreamland, and AEF Highland, amounting to US\$5.326 million.

15 Includes two emergency recovery credits of US\$50 million each, approved in 1995 and 1997, respectively, and fully disbursed.

16 Total approved amount, covering closed and active credits which also included cancellations. 


\section{Rwanda: Statistical Issues}

1. The economic and financial database of Rwanda is weak, in part due to the destruction caused by the 1994 war. Since then Rwanda has received considerable technical assistance in rebuilding the country's statistical database, and there has been some progress in the collection and reporting of economic and financial statistics. The authorities are fully cooperative in providing data to the Fund. Rwanda has very few statistical publications, but the authorities initiated in 1998 a new annual publication covering the main economic and financial data since 1990. National accounts and price statistics, government finance, and balance of payments statistics suffer from significant quality weaknesses. Monetary statistics are adequate for surveillance and program monitoring, but their quality needs to be further improved. Additional efforts are needed to improve statistics relevant for banking supervision.

\section{Real sector}

2. The Statistics Directorate of the Ministry of Finance and Economic Planning (MINECOFIN) prepares data on national accounts. After the 1994 war, the authorities compiled national accounts data starting from 1990. Nevertheless, the quality of these data is weak, reflecting shortages of human and material resources. While considerable effort was made to improve the reliability of GDP estimates using the production approach, significant weaknesses in data collection on expenditures and income remain. These weaknesses are reflected in uncertainties regarding the composition of GDP and they complicate, in particular, an adequate assessment of developments in savings and investment. The reliability of national accounts estimates are further hampered by weak external sector statistics. In 2003, an East AFRITAC mission visited Kigali to advise the authorities on real sector statistics issues, especially in the area of secondary sector statistics.

3. Monthly data on the consumer price index (CPI) are compiled by MINECOFIN. The National Bank of Rwanda (NBR) also compiles a CPI for Kigali. In 1996, the MINECOFIN started publishing a CPI for Kigali and urban and rural areas, based on a household incomeexpenditure survey (HIES) conducted between 1982 and 1986. However, the quality of the CPI is weak, as it does not reflect changes in household consumption patterns since the base period (March-June 1982). In 1996, a technical assistance mission from the Statistics Department proposed measures to improve the quality of the CPI, including the implementation of a new HIES. The HIES has recently been completed, and work is now under way to update the CPI basket weights with a view to compiling a single price index for Kigali. The introduction of the new CPI, planned to be available from January 2004, has not taken place by the end of April .

4. Data on employment and wages are not collected, except for the central government and for daily informal work. 
5. Real sector data are reported regularly for publication in International Finance Statistics (IFS), although with some lags, particularly for GDP estimates.

\section{Government finance statistics}

6. The authorities transmit to the African Department detailed monthly data on revenue and expenditure, with a lag of three-four weeks. These data are compiled by a flash-reporting unit, which was established in 1996 within MINECOFIN with the assistance of the Fund. A functional classification of government expenditure has been designed and was presented with the 2003 budget. Within the economic classification, expenditures belonging to areas identified by the PRSP as "priority areas" are clearly identifiable. The fiscal data do not capture consistently capital expenditure (almost entirely foreign) because capital projects are mainly carried out by line ministries outside the regular budget procedures. Efforts are under way to integrate the development budget into the normal budgetary procedures. Fiscal data have often shown a discrepancy between the deficit as recorded from above the line with that derived from below the line (i.e., its financing). Together with Fund staff, the authorities have made adjustments for changes in the balance of non-core government accounts, for changes in cash in vault at the revenue authority, for accounting errors and for other factors.

7. Selected aggregates on annual central government operations through 2002 have been reported to the Statistics Department and are published in IFS. These data exhibit large statistical discrepancies, mainly due to the time of recording of expenditures and the link with financing data. No sub-annual data are reported to STA and government finance statistics have been reported for the GFS Yearbook only through 1993.

\section{Monetary statistics}

8. The balance sheet of the NBR is transmitted to the African Department on a weekly basis with a lag of one week, and the monetary survey and the balance sheets of commercial banks are transmitted on a monthly basis with a lag of about four weeks. Detailed data on interbank money market transactions are also provided upon request to mission staff. The NBR established a working group to implement the recommendations of previous missions. A money and banking statistics mission visited Kigali in November 2000 and conducted an indepth review of the compilation of monetary statistics. The NBR is currently implementing the mission's recommendations, and has adopted in June 2002 an improved classification of the central bank balance sheet accounts, specifically those related to transactions with the government. Monetary data are reported separately to the Statistics Department on a timely basis and published in IFS. In early 2004, the NBR changed the reporting format for the banking sector balance sheet to the framework provided by the IMF MFS Manual.

\section{Balance of payments statistics}

9. Foreign trade data are provided to Fund missions by the NBR. There are inconsistencies between data reported by the NBR and data recorded by customs. There is also significant unrecorded trade, and information on invisible transactions is scarce. There are indications of significant under recording of official external transfers, and data on the 
level and composition of private flows are poor. One key challenge, highlighted in a also in the recommendations of a STA mission to Kigali in September 2000, still consists in ensuring an adequate number of well-trained staff for balance-of-payments compilation. Trade in goods data are mostly collected by customs with the major exception of imports and exports of electricity, tea, and coffee. Customs is expected to take over responsibility for the reporting of the latter goods in the near future. Another recommendation of the STA mission, the implementation of supplementary surveys, is currently being implemented: the NBR launched a unit in charge of these surveys, which, however, still lacks adequate training.

10. Annual balance of payments and quarterly import and export data are reported to STA, albeit with some lags, and are published in IFS. Rwanda sent methodology notes describing the compiling methods for balance of payments statistics which were included in Part 3 of the 2002 Balance of Payments Statistics Yearbook (BOPSY).

11. Databases on external public debt are maintained by both MINECOFIN and the NBR. The authorities have established a committee, composed of the staffs of the ministries of Finance and Economic Planning and Foreign Affairs and the NBR, to collect, harmonize, and monitor information on external public debt. 


\section{Rwanda: Core Statistical Indicators}

(As of April 28, 2004)

\begin{tabular}{|c|c|c|c|c|c|c|c|c|c|c|c|c|c|}
\hline & $\begin{array}{l}\text { Exchange } \\
\text { Rates }\end{array}$ & $\begin{array}{l}\text { International } \\
\text { Reserves 5/ }\end{array}$ & $\begin{array}{l}\text { Central } \\
\text { Bank } \\
\text { Balance } \\
\text { Sheet }\end{array}$ & $\begin{array}{l}\text { Reserve/ } \\
\text { Base } \\
\text { Money }\end{array}$ & $\begin{array}{l}\text { Broad } \\
\text { Money }\end{array}$ & $\begin{array}{l}\text { Interest } \\
\text { Rates }\end{array}$ & $\begin{array}{l}\text { Consumer } \\
\text { Price } \\
\text { Index }\end{array}$ & $\begin{array}{l}\text { Exports/ } \\
\text { Imports }\end{array}$ & $\begin{array}{l}\text { Current } \\
\text { Account } \\
\text { Balance }\end{array}$ & $\begin{array}{l}\text { Overall } \\
\text { Government } \\
\text { Balance 6/ }\end{array}$ & GDP/GNP & $\begin{array}{l}\text { External } \\
\text { Public } \\
\text { Debt 7/ }\end{array}$ & $\begin{array}{l}\text { Debt } \\
\text { Service } \\
7 /\end{array}$ \\
\hline $\begin{array}{l}\text { Date of Latest } \\
\text { Observation }\end{array}$ & Current & $4 / 16 / 04$ & $4 / 16 / 04$ & $4 / 16 / 04$ & $1 / 31 / 04$ & $1 / 04$ & $1 / 04$ & $7 / 03$ & 2002 & $3 / 04$ & $20031 /$ & $12 / 021 /$ & $12 / 031 /$ \\
\hline $\begin{array}{l}\text { Date } \\
\text { Received } \\
\end{array}$ & Current & $4 / 23 / 04$ & $4 / 23 / 04$ & $4 / 23 / 04$ & $3 / 29 / 04$ & $3 / 29 / 04$ & $3 / 29 / 04$ & $10 / 03$ & $2 / 03$ & $4 / 04$ & $2 / 04$ & $2 / 03$ & $4 / 04$ \\
\hline $\begin{array}{l}\text { Frequency of } \\
\text { Data }\end{array}$ & Daily & Weekly & Weekly & Weekly & Monthly & Monthly & Monthly & Monthly & Annually & Monthly & Annually & Annually & Annually \\
\hline $\begin{array}{l}\text { Frequency of } \\
\text { Reporting }\end{array}$ & Weekly & Weekly & Weekly & Weekly & Monthly & Monthly & Monthly & Monthly & $\begin{array}{l}\text { Semi- } \\
\text { annually }\end{array}$ & Monthly & $\begin{array}{l}\text { Semi- } \\
\text { annually }\end{array}$ & $\begin{array}{l}\text { Semi- } \\
\text { annually }\end{array}$ & $\begin{array}{l}\text { Semi- } \\
\text { annually }\end{array}$ \\
\hline $\begin{array}{l}\text { Source of } \\
\text { Update }\end{array}$ & NBR 2/ & NBR 2/ & NBR 2/ & NBR 2/ & NBR 2/ & NBR 2/ & NBR 2/ & NBR 2/ & NBR 2/ & MFEP 3/ & MFEP 3/ & MFEP 3/ & MFEP 3/ \\
\hline $\begin{array}{l}\text { Mode of } \\
\text { Reporting }\end{array}$ & $\begin{array}{l}\text { Facsimile/ } \\
\text { e-mail }\end{array}$ & $\begin{array}{l}\text { Facsimile/ } \\
\text { missions/ } \\
\text { e-mail }\end{array}$ & $\begin{array}{l}\text { Facsimile/ } \\
\text { missions/ } \\
\text { e-mail }\end{array}$ & $\begin{array}{l}\text { Facsimile/ } \\
\text { missions/ } \\
\text { e-mail }\end{array}$ & $\begin{array}{l}\text { Facsimile/ } \\
\text { missions/ } \\
\text { e-mail }\end{array}$ & $\begin{array}{l}\text { Facsimile/ } \\
\text { missions/ } \\
\text { e-mail }\end{array}$ & $\begin{array}{l}\text { Facsimile/ } \\
\text { missions/ } \\
\text { e-mail }\end{array}$ & $\begin{array}{l}\text { Facsimile/ } \\
\text { missions/ } \\
\text { e-mail }\end{array}$ & $\begin{array}{l}\text { Mainly } \\
\text { during } \\
\text { missions }\end{array}$ & $\begin{array}{l}\text { Facsimile/ } \\
\text { missions/ } \\
\text { e-mail }\end{array}$ & $\begin{array}{l}\text { Mainly } \\
\text { during } \\
\text { missions }\end{array}$ & $\begin{array}{l}\text { Mainly } \\
\text { during } \\
\text { missions }\end{array}$ & $\begin{array}{l}\text { Mainly } \\
\text { during } \\
\text { missions }\end{array}$ \\
\hline Confidentiality & No & $4 /$ & $4 /$ & $4 /$ & $4 /$ & No & No & $4 /$ & $4 /$ & $4 /$ & 4/ & 4/ & 4/ \\
\hline $\begin{array}{l}\text { Frequency of } \\
\text { publication }\end{array}$ & Monthly & Monthly & Monthly & Monthly & Monthly & Monthly & Monthly & Annually & Annually & Annually & Annually & Annually & Annually \\
\hline
\end{tabular}

1/ Estimates.

2/ NBR = National Bank of Rwanda.

3/ MFEP = Ministry of Finance and Economic Planning

4/ Preliminary data for staff use only; actual data are unrestricted.

5/ International Reserves refer to gross official reserve assets.

6/ The category Overall Government Balance refers to Central Government Balance.

7/ External public debt refers to external public debt owed or guaranteed by the central government, local governments or the National Bank of Rwanda. Normal importrelated credits are excluded. The term "debt" has the meaning set forth in point No. 9 of the Guidelines on Performance criteria with respect to foreign debt adopted on

August 24, 2000. 
Rwanda: Work Program-Proposed Phasing of Disbursements Under the

PRGF Arrangement, 2002-05

\begin{tabular}{|c|c|c|}
\hline \multirow[t]{2}{*}{ Date } & \multirow{2}{*}{ Description } & Amount \\
\hline & & $\begin{array}{c}\text { (In millions } \\
\text { of SDRs) }\end{array}$ \\
\hline
\end{tabular}

August 12, 2002 Three-year arrangement approved

August 16, 2002 First disbursement

0.574

0.7

June 13, $2003 \quad$ First review completed

June 14, $2003 \quad$ Second disbursement

0.571

June-04

Second and third review completed

June-04

Third and fourth disbursement

1.142

November-04 Fourth review completed

November-04 Fifth disbursement

0.571

May-05

Fifth review completed

May-05

Sixth disbursement

0.571

0.7

November-05 Sixth review completed

November-05 Seventh disbursement

0.571 


\section{Rwanda: Safeguards Assessment-Summary of Conclusions}

A safeguards assessment of the National Bank of Rwanda (NBR) was completed in April 2003. The assessment received full cooperation of the NBR, and an agreement was reached on a set of measures to remedy identified vulnerabilities.

\section{Vulnerabilities}

The vulnerabilities identified included the following:

(i) The external audit mechanism needs strengthening to ensure that annual external audits are conducted and the Committee of Auditors becomes fully operational

(ii) The newly established internal audit department requires additional resources to become operational

(iii) Procedural controls over the compilation of program monetary data require strengthening, and

(iv) Formal investment guidelines for the management of foreign reserves need to be established

\section{Remedies under program conditionality}

On the basis of the safeguards assessment, the National Bank of Rwanda developed an action plan to strengthen external and internal audit mechanisms, legal structure and independence, financial reporting and internal controls. Since then, of seventeen recommendations by the Safeguards Assessment mission, eleven have been implemented. In particular, a regular and yearly external audit system has been established, with the 2003 audit expected before end (March 2004); the foreign exchange reserve management system has been overhauled, including through the establishment of investment guidelines and training of staff; the bank discontinued the distribution of unrealized foreign exchange gains; and financial reporting has been improved with the production of consistent monetary data that is reported to the Fund and that contained in the NBR's financial statements. Furthermore, the internal audit department has been operationalized, with the recruitment of qualified staff and with technical assistance from the Fund, and significant progress has been made in implementing the recommendations of the 2002 external audit exercise. 


\section{Rwanda: Millennium Development Goals}

\begin{tabular}{|c|c|c|c|c|}
\hline & 1990 & 1995 & 2001 & 2002 \\
\hline \multicolumn{5}{|c|}{$\begin{array}{l}1 \text { Eradicate extreme poverty and hunger }(2015 \text { target }=\text { halve } 1990 \text { US } \$ 1 \text { a day poverty and } \\
\text { malnutrition rates) }\end{array}$} \\
\hline Population below $\$ 1$ a day $(\%)$ & .. & .. & .. & .. \\
\hline Poverty gap at $\$ 1$ a day $(\%)$ & .. & .. & .. & .. \\
\hline Percentage share of income or consumption held by poorest $20 \%$ & .. & .. & .. & .. \\
\hline Prevalence of child malnutrition ( $\%$ of children under 5 ) & 29.4 & 27.3 & 24.0 & .. \\
\hline Population below minimum level of dietary energy consumption (\%) & 34.0 & .. & 40.0 & .. \\
\hline \multicolumn{5}{|c|}{2 Achieve universal primary education (2015 target $=$ net enrollment to 100 ) } \\
\hline Net primary enrollment ratio ( $\%$ of relevant age group) & 65.9 & .. & 97.3 & .. \\
\hline Percentage of cohort reaching grade $5(\%)$ & 60.0 & .. & 39.1 & .. \\
\hline Youth literacy rate (\% ages $15-24)$ & 72.7 & 78.6 & 84.2 & 84.9 \\
\hline \multicolumn{5}{|l|}{3 Promote gender equality (2005 target $=$ education ratio to 100 ) } \\
\hline Ratio of girls to boys in primary and secondary education (\%) & 97.6 & .. & 97.1 & .. \\
\hline Ratio of young literate females to males (\% ages $15-24)$ & 86.4 & 91.8 & 96.3 & 96.9 \\
\hline Share of women employed in the nonagricultural sector $(\%)$ & .. & .. & & .. \\
\hline Proportion of seats held by women in national parliament (\%) & 17.0 & 17.0 & 26.0 & 26.0 \\
\hline
\end{tabular}

4 Reduce child mortality (2015 target $=$ reduce 1990 under 5 mortality by two-thirds)

$\begin{array}{lllll}\text { Under } 5 \text { mortality rate (per 1,000) } & 178.0 & 210.0 & 183.0 & 191.0 \\ \text { Infant mortality rate (per 1,000 live births) } & 107.0 & 122.0 & 96.0 & 99.0 \\ \text { Immunization, measles (\% of children under 12 months) } & 83.0 & 84.0 & 78.0 & . .\end{array}$

5 Improve maternal health $(2015$ target $=$ reduce 1990 maternal mortality by three-fourths)

Maternal mortality ratio (modeled estimate, per 100,000 live births) $\quad . . \quad 2,300.0 \quad$..

$\begin{array}{llll}\text { Births attended by skilled health staff }(\% \text { of total }) & 25.8 & . . & 30.8\end{array}$

6 Combat HIV/AIDS, malaria and other diseases (2015 target $=$ halt, and begin to reverse, AIDS, etc.)

Prevalence of HIV, female (\% ages 15-24)

Contraceptive prevalence rate (\% of women ages $15-49)$

Number of children orphaned by HIV/AIDS

Incidence of tuberculosis (per 100,000 people)

Tuberculosis cases detected under DOTS (\%)

$\begin{array}{lll}. . & . . & 11.2 \\ 21.0 & . . & 13.2 \\ . . & . . & 260,000 \\ . . & . & 404.7 \\ . . & 32.0 & 29.0\end{array}$

7 Ensure environmental sustainability $(2015$ target $=$ various $)$

Forest area (\% of total land area)

Nationally protected areas (\% of total land area)

GDP per unit of energy use (PPP dollars per $\mathrm{kg}$ oil equivalent)

$\mathrm{CO} 2$ emissions (metric tons per capita)

Access to an improved water source (\% of population)

Access to improved sanitation ( $\%$ of population)

Access to secure tenure ( $\%$ of population)

$\begin{array}{llll}18.5 & . . & 12.4 & . . \\ . . & 14.6 & 14.7 & 14.7 \\ . . & . & . & . . \\ 0.1 & 0.1 & 0.1 & . . \\ . . & . . & 41.0 & . . \\ . . & . . & 8.0 & . . \\ . . & . . & . . & . .\end{array}$

8 Develop a Global Partnership for Development (2015 target = various)

Youth unemployment rate (\% of total labor force ages 15-24)

Fixed line and mobile telephones (per 1,000 people)

Personal computers (per 1,000 people)

Source: World Bank. 


\section{Statement by the IMF Staff Representative \\ June 9, 2004}

This statement provides information on economic developments that became available since the issuance of the staff report on the Second and Third Reviews under Rwanda's Three-Year Arrangement under the PRGF. The new information does not alter the thrust of the staff appraisal.

\section{Prior Actions}

1. The Second and Third Reviews of the PRGF-supported program for 2004 set the following prior actions, to be taken by May 31, 2004: (i) cabinet approval of a new procurement code; (ii) central bank (NBR) establishment of written procedures ensuring that data reported to the Fund for program purposes are consistent with the TMU and reconciled with accounting records; (iii) restoration, by the NBR, of the regulation on the net open foreign exchange position of commercial banks to the text in force in June 2003.

2. All three prior actions were met on time. A new procurement code was approved by cabinet on May 31, 2004. The code, which addresses issues raised in the Auditor General's report for 2002, strengthens transparency in the award of government contracts and widens eligibility for participation in tenders for the provision of goods and services for public sector entities. Statistical guidelines and verification procedures meeting the prior action on written procedures were issued by the central bank on May 31, 2004 and will be applied beginning with the submission of data for April 2004. The revised regulation on the net open foreign exchange position of commercial banks, which brings the regulation into line with widely observed international norms, took effect on May 19, 2004.

\section{Recent Economic Developments}

3. Rainfall through April 2004 was close to the long term average, and agricultural output for this year could support the targeted real GDP growth rate of 6 percent envisaged in the program. The 12-month rate of inflation peaked at 11.2 percent in February 2004, before declining to 10.4 percent in April. As the value of the Rwanda franc vis-à-vis the US dollar stabilized in 2004 following a pronounced depreciation during 2003, the price pass-through of depreciation should moderate in the period ahead.

4. The government's operations through end-April 2004 were consistent with the program targets. Revenue was slightly above the projected level, boosted by strong customs collections. Domestic spending and the domestic fiscal balance remained in line with program targets. The stock of unpaid government bills ("arrears") accumulated during 2003 was cleared, as of end-May 2004.

5. The end-2003 liquidity overhang was progressively reduced through April 2004 and net credit from the banking system to government was on track. In line with program 
objectives, commercial bank reserves were brought substantially in line with requirements and, as of May 21, 2004, the year-on-year growth rate for reserve money had declined to 10.3 percent. Central bank foreign exchange sales have been limited, in line with the program.

6. Five interested investors have now completed due diligence studies of the Commercial Bank of Rwanda. Investor bids are due on June 16, 2004.

7. The central bank issued a payment order on June 7, 2004, to fully amortize the external government-guaranteed loan for the hotel project. 


\section{Statement by the IMF Staff Representative \\ June 9, 2004}

The following information responds to questions posed by Executive Directors on the report submitted by the staff.

\section{Realism of the program}

Macroeconomic developments thus far in 2004 have been encouraging: good rains appear to provide a basis for a recovery in agricultural production; inflation has begun to moderate; and the international market price for coffee has strengthened in line with the program projections. As noted in the staff statement, the performance of government revenue and spending during January-April 2004 provide support for the authorities' commitment to the 2004 program.

\section{Growth prospects}

For 2004, projected GDP growth largely reflects the expected recovery of agricultural production, following a drought-induced decline in 2003. Regarding the medium- to longterm, Rwanda's growth strategy has benefited from extensive technical assistance, including from the World Bank, EU, AfDB, and several bilateral partners. The strategy, which is subject to periodic review, relies on improvements in agricultural productivity. As growth prospects depend on the effective implementation of the strategy, the authorities will need to make a strong effort to see that it is realized.

\section{Corrective Measures}

To correct for slippages under the 2003 program, the 2004 program includes a substantial reduction in net credit to government from the banking system and a fiscal program that incorporates a specific contingency mechanism to assure that macroeconomic objectives will be achieved. Domestic arrears accumulated during 2003 were cleared by end-May 2004. Through end-May, reserve money growth, reflecting the limited extension of net banking system credit to government, was lower and the net foreign assets of the central bank higher than had been targeted. The financing terms for the energy rehabilitation project have been modified, bringing them into compliance with the floor on concessionality set under the program. On the structural side, legislation on the new budget law, financial instructions, and new tax legislation have been readied for submission to parliament.

\section{Fiscal issues}

Fiscal consolidation: The government's fiscal program for 2004 targets progress towards the achievement of the millennium development goals, in line with policy frameworks that have been developed with the assistance of the World Bank, the AfDB, EU and bilateral donors. While total expenditure is programmed to rise substantially, owing to higher grants, changes to the structure of recurrent expenditure are limited. Value added and customs tax rates were 
revised during 2002-03. Revenue enhancement will result from the implementation of the revised tax and customs laws and improvement in tax administration, with technical assistance from the Fund and other partners.

Wage bill and civil service reform: The wage bill, at 4.8 percent of GDP, is among the lowest in Africa. Looking forward, the authorities have signaled their intent to reform the civil service, in cooperation with partners, with the objective of implementing best practices in this area.

Composition of expenditure: Fiscal operations are recorded in detail in the government's computerized budget and expenditure system. Monthly data on spending authorizations, commitments, and payments are communicated to the Fund in detail, with a four week lag, including military outlays for wages and goods and services. The Auditor General's report on the audit of the Ministry of Defense for fiscal 2001 and 2002 noted that defense spending was funded through budget allocations disbursed through the system used for reporting to the Fund. In 2003, defense spending on wages and goods and services decreased to 2.7 percent of GDP, from 2.9 percent in 2002. Fund staff would welcome any other reliable information regarding defense spending that Directors could provide.

Contingency measures to deal with a possible shortfall in aid inflows: Reflecting uncertainties regarding external program assistance, the government has identified a list of contingent outlays for reduction, which consist mainly of delaying funding increases for districts and the introduction of new programs in nonessential areas.

\section{Exports}

Performance versus projections: Exports were projected by the staff using WEO prices. However, year-to-year supply shocks have resulted in a pronounced variability in international market prices for coffee and tea. Recent volume decreases reflected, in part, a drop in international prices below production costs.

Medium-term outlook: For 2004, the projected volume increase for coffee ( 2 percent) is conservative. In addition, data on tea exports for January-March 2004 are in line with projected volumes for these exports. The realism of the medium- to long-term projections depends critically on the implementation of Rwanda's export promotion action plan, which is being developed with the assistance of the World Bank. Reflecting the importance attached by the staff to this issue, a structural benchmark for cabinet approval of the action plan was established for end-September 2004.

Mineral exports: Regarding coltan, Rwanda's exported 732 tons of this mineral during 2003 , with a value of US\$6.4 million. Production is projected to decline by another 30 percent in volume terms during 2004. The staff is not aware of any claims regarding the origin of Rwanda's coltan exports in 2003. If any additional information is made available, the staff will pursue the matter during discussions for the forthcoming program review. 


\section{Structural conditionality and capacity constraints}

The structural conditionality set for Rwanda's key economic legislation targeted the creation of a comprehensive and coherent framework. These reforms were considered to be fundamental to improving economic efficiency, strengthening the government finances, and enhancing accountability.

As the authorities' human resources were limited, the realization of the performance criteria depended on the coordinated scheduling of supporting technical assistance which, at times, was delayed. Nonetheless, critical progress has been achieved in all of the targeted areas: a new procurement code has been approved, and the budget law, financial instructions, and tax and customs laws have been finalized and readied for submission to parliament. Given the advanced stage reached in implementing these reforms, new performance criteria were not seen by the staff as being necessary. Taking into account the need to streamline conditionality, the staff was of the view that it was important to focus on other measures that were necessary to push the reform agenda forward. The staff will continue to monitor the implementation of the structural performance criteria that were not observed and their status will be reported in subsequent reviews.

\section{Banking statistics}

The commercial banking statistics reported to the Fund correspond to data collected by the central bank as part of its regular supervision activities. In the past, these data have allowed the staff to identify emerging issues in some institutions. While the data reports are detailed, their communication does not create additional work for the authorities. 
Press Release No. 04/114

FOR IMMEDIATE RELEASE

June 10, 2004
International Monetary Fund

Washington, D.C. 20431 USA

\section{IMF Completes Second and Third Reviews Under Rwanda's PRGF Arrangement, Approves US\$1.68 Million Disbursement, and Grants Additional Interim Assistance of US\$6.6 Million Under the Enhanced HIPC Initiative}

The Executive Board of the International Monetary Fund (IMF) has completed the second and third reviews of Rwanda's economic performance under the Poverty Reduction and Growth Facility (PRGF) arrangement. As a result, Rwanda will be eligible to draw an amount equivalent to SDR 1.14 million (about US \$1.68 million), bringing the total amount disbursed under the arrangement to SDR 2.29 million (about US\$3.38 million).

The Board also reviewed Rwanda's Annual Progress Report on Poverty Reduction Strategy Paper and concluded that it provides a sound basis for continued access to Fund concessional financial assistance. In addition, the Executive Board approved additional interim assistance under the enhanced Heavily Indebted Poor Countries (HIPC) Initiative of SDR 4.482 million (about US\$6.6 million) through June 8, 2005.

In completing the reviews, the Board granted waivers for the nonobservance of quantitative performance criteria for end-June 2003 and end-December 2003, and structural performance criteria for end-December 2003, end-January 2004 and end-March 2004.

Rwanda's PRGF arrangement was approved on August 12, 2002 (see Press Release No. 02/36), for SDR 4 million (about US\$6 million).

The PRGF is the most concessional facility for low-income countries. PRGF-supported programs is based on country-owned poverty reduction strategies adopted in a participatory process involving civil society and development partners, and articulated in a Poverty Reduction Strategy Paper (PRSP). This is intended to ensure that PRGF-supported programs are consistent with a comprehensive framework for macroeconomic, structural, and social policies to foster growth and reduce poverty. PRGF loans carry an annual interest rate of 0.5 percent and are repayable over 10 years with a $5 \frac{1}{2}$-year grace period on principal.

In commenting on the Executive Board's discussion on Rwanda, Agustín Carstens, Deputy Managing Director and Acting Chair, stated: 
"The wide range of challenges faced by Rwanda in 2003, including the adoption of a new constitution and national elections, stretched its limited capacity. Macroeconomic performance and policy implementation fell short of the standards that had been maintained in preceding years. However, the authorities' renewed commitment to the program's objectives has been accompanied by some improvements in economic indicators in 2004.

"With the political transition completed, strengthened policy implementation will be needed to achieve the program's objectives during 2004. Under the program, real GDP growth is expected to accelerate to 6 percent, while monetary policy will aim at limiting inflation to 5 percent by year's end. Fiscal policy will center on consolidating recent gains in revenue mobilization and take advantage of increased external budgetary support to address critical social needs, including free primary schooling and extended health services, in line with the Poverty Reduction Strategy Paper. Spending under the program will be carefully monitored to ensure transparency and tightened management of public resources, as well as the appropriate funding of priority sectors, and will be complemented by a strengthening of procedures for public procurement. In addition, given the high debt burden the authorities will limit new external borrowing and promote higher domestic savings.

"Structural policies will focus on promoting growth by encouraging private investment, improving the legal framework, and strengthening public financial administration. Priority will also be given to privatization of state enterprises - including in the telecommunications, tea, banking, and hotel sectors - export promotion, and addressing weaknesses in financial sector supervision. It will be essential that the authorities work closely with external and domestic partners to achieve these objectives, tackle the serious capacity constraints facing Rwanda, and improve social conditions. In addition, growth and social progress will require Rwanda's continued commitment to securing a lasting peace in the Great Lakes area," Mr. Carstens said. 


\section{Statement by Damian Ondo Mañe, Executive Director for Rwanda and Laurean W. Rutayisire, Alternate Executive Director \\ June 9, 2004}

\section{I - Introduction}

Our Rwandese authorities would like to express their appreciation to Fund management and staff for their continuous support. They are grateful for the continued assistance received from the international community in their efforts towards fighting poverty and achieving the MDGs. Since the outset of the current Fund-supported program, Rwanda has made remarkable achievements in maintaining macroeconomic stability, achieving appreciably high growth, increasing social sector spending and remaining current on external debt repayment. Also up to June 2003, all quantitative performance criteria were met.

All these achievements have been made in spite of Rwanda's unusual experience of genocide in 1994 and its complex socio economic aftermath. As part of dealing with this situation, the government had to carry out in 2003 the political institutional building process, which would lay a solid foundation for a democratic society, good governance and rule of law. This involved carrying out referendum for the adoption of a new constitution, presidential and parliamentary elections and putting in place democratic institutions required by the new constitution including establishment of the Gacaca Court to try perpetrators of genocide. This process was successfully carried out. However, it entailed an additional unforeseen cost financed entirely by domestic resources in the absence of external assistance. Consequently, some performance criteria under the PRGF for the second half of year 2003 were missed.

Since that time, our authorities have been working with Fund staff to put in place corrective measures. During the first quarter ending March 2004, our authorities have implemented those measures, and quantitative targets have been brought in line with the program criteria and they are fully committed to maintaining the momentum. To this effect, the measures agreed on with staff, as prior actions for the second and third review, have been implemented. In view of the corrective measures taken, the commitment to meeting the program's objectives and the record of accomplishment, our Rwandese authorities request waivers for the nonobservance of the missed performance criteria, approval of the completion of the second and third review under the PRGF and additional assistance under the enhanced HIPC Initiative.

\section{II - Recent Economic Developments}

As an economy predominantly based on peasant agriculture, Rwanda's real GDP continues to be affected by the vagaries of weather. Thus, although a higher GDP growth rate of 9.2 was achieved in 2002; in 2003, the growth rate decelerated sharply to 0.9 following severe drought that affected agricultural production. Also, owing to the fall in agricultural output inflation rose to 7.7 percent at the year-end against the program target of 3 percent and 6.2 percent in 2002. 
However, the authorities have adopted a number of measures to increase productivity in agricultural sector. Those measures include land terracing, reforestation, road rehabilitation and extension, improving access to rural credit and the market channels for the distribution of fertilizer as well as improved seeds.

In the fiscal area, fiscal performance through end-June was broadly on target, although during the second half of 2003 the fiscal position weakened. Exceptional expenditure related to Rwanda's unique post genocide situation, mentioned earlier above, accounted for the widening of the domestic fiscal deficit. Hence, the domestic fiscal deficit in 2003 could not meet the objective of 2.6 percent of GDP set out in the program. This was in spite of the fact that revenue performance continued to be stronger than programmed. The persistent improvement in revenue collection is attributed to the revenue enhancement measures implemented since 2002. These measures include improvement in direct tax collection and strengthening Rwanda Revenue Authority.

On the expenditure side, other than the exceptional increase mentioned above viz, expenditure on new constitution, presidential and parliamentary elections and putting in place democratic institutions required by the new constitution other expenditures remained as budgeted. In their efforts to redress the situation and streamline the public finance management, the authorities have appointed a treasury management team and have initiated movement towards unified treasury account and reinforce control through the Auditor General's role and the establishment of an internal audit Department within the Ministry of Finance. Moreover, as a measure to reinforce government expenditure control, the prior action which required cabinet approval of a new procurement code has been implemented. As result of these measures, both revenue and expenditure performances as at end-March 2004 have been in line with end-June performance criteria.

On the monetary front, net credit to government was higher than program target owing to exceptional government expenditure increase which was financed by bank borrowing in the absence of the expected external financing. There was also an increase in private sector credit notably as a result of commercial banks lending to Prime holdings hotel project. Owing to the overall increase in domestic credit, reserve and broad money were above the program targets. In order to mop up the excess liquidity, the Central bank sold foreign exchange, which in addition to the drop of export proceeds and delayed donor budget support assistance led to decline in the foreign exchange position, hence missing the program target.

In the financial sector, progress has been achieved, notably in loan recovery, information sharing on credit risk, on-site banking supervision and creation within the Central Bank of a micro Finance Sector Supervision Department. Moreover, the plan for the restructuring of Caisse Hypothécaire du Rwanda is underway and substantial progress towards the sale of Banque Commerciale du Rwanda has been made. Nevertheless, challenges remain as during the second half of the year, some banks failed to comply with the prudential norms with respect to the minimum reserve requirements and foreign exchange open position. In addition, capacity constraints have continued to limit the effectiveness of bank supervision. 
As regards the structural area, some performance criteria were missed at the test date. However, as well noted by the staff, there has been substantial progress as some of the structural reforms have already been implemented, while others are in the advanced stage of being implemented.

In the external sector, the current account slightly deteriorated in 2003 compared to the target due to fall in coffee and Coltan export volumes as well as weak international prices for coffee and tea. The decline in exports has led to Rwanda's net present value of debt ratio to stand at around 300 percent at end 2003 against 190 based on the decision point projection. In order to strengthen the export performance, the authorities have put in place an export promotion strategy with the assistance of the World Bank. With respect to the external debt, the authorities continued to strengthen its management through implementing an action plan designed in this regard. To this effect, the second phase of a capacity-building program for debt management was started in January with the assistance of Debt Relief International. In addition, performance criterion for non-accumulation of external arrears in 2003 was met. Also external borrowings have been kept in line with the level projected in the HIPC decision point document.

\section{III - Medium Term Program and Policies for 2004}

For the medium term, the strategy for poverty reduction and economic growth will be guided by the set of policies contained in the Poverty Reduction Strategy Paper issued in June 2002. The progress report issued last July indicated progress made in human resource development and achievements recorded in governance improvement.

Our Rwandese authorities recognize the critical importance of macroeconomic stability towards the attainment of poverty eradication objectives as well as the reduction of macroeconomic imbalances and dependence on external assistance. In order to achieve these objectives, the authorities intend to improve public finances, strengthen the financial sector and implement the poverty reduction strategy through strong actions in rural development, human resources and physical infrastructure development. Based on this strategy and supported by our authorities' strong commitment, the macroeconomic targets for the medium term are to achieve a real GDP growth of at least 6 percent; contain inflation at 5 percent in 2004 and 3 or 4 percent thereafter and reconstitute gross international reserves to at least six moths of imports.

\section{Fiscal Policy}

In accordance with the stated medium term objectives, the fiscal program for 2004 seeks to consolidate gains made in revenue policy and administration over the past 2 years. To this effect, based on the technical assistance from bilateral and multilateral partners further steps to strengthen revenue administration will be taken. Moreover, the reforms already made in direct tax involving revision of marginal rates and taxation of professional remuneration will be fully implemented. Reforms in the Rwanda Revenue Authority to establish a large taxpayer unit will also be put in place. 
On the expenditure side, the core outlays will remain within the program targets while increasing slightly. This increase reflects expenditure for newly constitutionally mandated institutions, free basic education, government contributions to HIV/AIDS programs, human resources development, tertiary education, labor-intensive works and export promotion. In the event of revenue shortfall as budgetary external assistance is uncertain the government stands ready to cut non-priority expenditure.

\section{Monetary Policy and Financial Sector Development}

The monetary policy objectives are to contain inflation under 5 percent at end 2004 and build up the international reserves and observe the broad money target set out for the year-end. As the authorities are determined to ensure that fiscal and monetary policies operate effectively, the Central Bank and Ministry of Finance will closely coordinate their actions through the newly established Treasury Committee.

Based on their fiscal objectives and targets for domestic credit growth as well as expected inflows of external financing, the authorities will continue to reconstitute the gross foreign reserves. Moreover, the operation on the foreign exchange market will be further strengthened. The NBR has also implemented the prior action, which requires that the regulation on the net open foreign exchange position of commercial banks be restored to the text in force in June 2003. Equally importantly, a review of the current auction system is envisaged in order to ensure that auction exchange rates are determined based on the market rules.

As regards the financial sector, our authorities will reinforce the supervision conducted by the central bank through its efforts to reestablish the mandatory compliance with prudential regulations. In this regard, the NBR will closely work with commercial banks on action plan to bring the banks to fully observe the banking regulations and requirements by the end of 2004. In the same vein, full annual audits of four banks are to be carried out in 2004 and as from 2005; the audit of all commercial banks will be conducted on an annual basis. In addition, the authorities will continue to execute the pending structural reforms in the financial sector including the restructuring of Caisse Hypothécaire du Rwanda and privatization of BCR. In their further endeavors to carry out the structural reforms in the financial sector, our authorities look forward to the forthcoming FSAP mission which will be jointly conducted by the Bretton Woods institutions with the objective to provide a comprehensive program for the reform and development of the financial sector.

\section{Structural reforms}

The main objectives set out in the structural area, namely to improve economic productivity, governance, efficiency and external sustainability will be intensified in 2004 in order to reflect priorities outlined in the PRSP. The privatization of the telecommunication enterprise and some tea factories will also be completed. In order to develop a conducive environment for private investment, the legal framework will be further strengthened through additional measures. In this regard, a new investment code as well as a new mining code are slated for consideration by the Parliament before year-end. 
As regards the external sector, an export promotion task force was put in place in December 2003 and a detailed export promotion action will be submitted before end-June for consideration by the authorities.

Regarding public finances, the achievements made during the past 2 years in establishing modern, efficient and transparent laws and institutions will be extended in 2004. In this regard, the new organic budget law, a draft tax law, a new procurement code and a draft customs law are in the process to be submitted to the Parliament for consideration. An inventory of the physical assets belonging to the public sector and joint development projects will be completed before end-2004 and annexed to the 2005 budget. These actions and new regulations reflect the authorities' commitment to further reinforce the legal framework governing the management of public finances.

\section{Poverty Reduction}

Our authorities are strongly determined to pursue the fight against poverty through a strategy that includes the private sector and rural led growth. The PRSP fully endorsed by all stakeholders has identified six priority areas on which to focus projects and programs for poverty reduction. The first progress report covering the period August 2002-June 2003 highlighted commendable achievements in health and education sectors as well as governance and infrastructure development.

The authorities are fully aware that the attainment of the poverty reduction objectives will be assured if sectorial strategies and programs in other sectors are similarly articulated and implemented. For this purpose, they will continue to use the cluster framework developed with the help of the World Bank and other donors as coordination mechanisms in articulating strategies for the remaining sectors.

\section{IV - Conclusion}

Under the Fund-supported programs, Rwanda has implemented sound macroeconomic policies and achieved remarkable economic and financial performances. In spite of the progress made so far, there remain a number of difficulties and challenges that need to be tackled in order to boost economic growth and stimulate economic diversification, and above all reduce poverty. Our authorities are strongly determined to capitalize up on past progress and persevere in their reforms and adjustment endeavors so that they can reach the completion point under the Enhanced HIPC initiative, which will further strengthen their efforts.

Our Rwandese authorities are hopeful that they can rely on additional international assistance to support their adjustment efforts. In this context, they would also like to renew their appreciation to the entire international community and the Fund in particular for their solidarity during the commemoration of the $10^{\text {th }}$ anniversary of the genocide in Rwanda. Given the authorities' commitment to pursue and deepen their adjustment efforts through the Fund-supported program, we would like, once again to request the Board approval for 
waivers of the performance criteria missed and completion of the second and third review under the PRGF, as well as for the provision of additional assistance under the Enhanced HIPC initiative. 